\title{
Radiology for Maxillofacial Surgeons: The Essentials
}

\author{
Shyamsundar K. Joshi and Annie I. Kochuveettil
}

\section{Learning Objectives}

- To identify the strategies in building an effective working relationship with the imaging department and help plan appropriate imaging investigations.

- To understand the principles of various imaging techniques; the advantages and disadvantages of these techniques in varying clinical scenarios.

- To gain awareness about the benefits and risks of using ionizing radiation and understand the basic principles of radiation protection.

- To establish the role of imaging as a treatment guide following different maxillofacial surgeries.

\subsection{Introduction}

Accurate diagnosis is the mainstay of proper surgical management in a maxillofacial surgical patient. Definitive diagnosis may be possible in a few patients based on clinical evaluation in the outpatient department or emergency setup. Other cases may require the use of supplementary investigations, including radiological imaging.

Like other medical specialties, the scope of Radiology is vast, playing a significant role in disease management. Penning a chapter on Radiology for a clinical branch-like Maxillofacial surgery is an uphill task. As a clinician, the maxillofacial surgeon, after having made the diagnosis, has the desire to visualize and gain in-depth knowledge of the ongoing disease process within the patient. Radiology plays an important supporting role in helping the clinician visualize within the patient's body. It is hence necessary for clinicians to have basic knowledge of the key concepts of

S. K. Joshi $(\triangle)$ - A. I. Kochuveettil Department of Radiology, SDM College of Medical Sciences and Hospital, Shri Dharmasthala Manjunatheshwara University, Dharwad, India
Radiology, helping them understand its applications in the diagnosis of a patient's clinical condition.

The scope of practice of a practicing MFS includes both surgical and nonsurgical problems that affect the orofacial region, and hence they need to be well versed with the imaging concepts of the head and neck region.

\section{Head and Neck Imaging}

Anatomy of the head and neck is complex and imaging in this region poses several challenges and an MFS needs to be cognizant of them.

Modern imaging has the unique advantage of being able to:

- Demonstrate intricate and critical anatomical structures packed in small compact space pockets.

- Define the anatomy pertinent to the region of clinical interest.

- Define the critical relation to vital structures like the brain and cerebral vessels.

- Define the related adjacent critical areas like the airway, orbital contents, and both intra- and extracranial neurovascular elements.

- Define the underlying pathological process.

Conventional Radiography is one of the modalities that have been available from early days, ever since X-rays were discovered by Wilhelm Conrad Roentgen in 1895 . However, it is regarded as a poor choice for anatomical evaluation due to overlapping of anatomical structures especially in the head and neck region. Yet it still has a popular role in its own humble way. It is universally available, the least timeconsuming imaging investigation, and gives a 'bird's eye view' of the region of interest. It provides instant practical 
information on the status of the airway and osseous elements, helps inferring the underlying pathological process based on the presence of air pockets or bony destruction, and at times may even reflect systemic pathology. It guides the clinician as to the further steps to be taken for patient management or to the need for further imaging. Despite it being the era of advanced technology, conventional radiography should invariably be the first choice among imaging investigations. In cases of complex maxillofacial injuries and polytrauma, when the patient's clinical status demands the next level of investigation, CT may be performed first, bypassing conventional radiography.

Pioneers in the field of imaging had a yearning to develop modalities that could demonstrate finer anatomical details in the region of interest.

This, coupled with the technological evolution, has paved the way for the development of various radiological imaging technologies that include:

- Ultrasonography (USG)

- Color Doppler

- Computerized Tomographic (CT) scan

- Dentascan

- Cone beam CT

- PET scans

- Radioisotope scanning

- Magnetic Resonance Imaging (MRI)

- Functional imaging, etc.

These advances in imaging techniques have enabled the MFS to diagnose with greater confidence and refine their approach to surgical treatment. This has also led to a reduction in unexpected surprises springing up on the surgical table.

Considering that a range of imaging modalities are available, it is imperative that the MFS has basic knowledge about their functionality, being familiar with the advantages and disadvantages of each so that the appropriate imaging investigation that gives the most optimal information in a given clinical setup can be selected. The rationale behind choosing the right investigation is that it should be easily available, least time consuming, cause least possible discomfort to the patient and be economical. Appropriate use of the concerned imaging modality should provide the maximum possible anatomical and functional information. This is possible if a genuine effort is made by an MFS to understand the basic principles and other related components of specific diagnostic imaging relevant to his field of interest and establish a close rapport with the imaging department.

\subsection{Requesting an Imaging Investigation}

Prior to requesting a radiological investigation, the MFS needs to have a clear idea about the usefulness of an investigation, its limitations, and whether it will help to improve the patient outcome. A radiological imaging requisition must specify the choice of investigation and the surgeon's expectations from it, so that the information received strengthens his approach in treating the patient.

It must provide mandatory demographic and relevant clinical data, including the patient's symptoms, which primarily brought him to the MFS, their duration, clinical signs, and the clinician's differential diagnosis of the same. In addition to concentrating on the clinical data, entries made available through the Hospital Information system (HIS) and the Radiology Information system (RIS), accurate patient identification by the MFS is essential prior to referral to the imaging department.

\subsection{Role of the Maxillofacial Surgeon (MFS) Vs. the Radiologist's expectation from the MFS}

An MFS deals with patients suffering from maxillofacial disease processes, but being a clinician primarily he is expected to have basic knowledge of various systemic clinical problems as well. Systemic disease may manifest as disease in the maxillofacial region or vice versa. For instance, a cystic lesion in the jaw could be the primary radiographic finding in a patient suffering from hyperparathyroidism. Similarly, patients suffering from systemic diseases like osteopetrosis, fluorosis, fibrous dysplasia, and other marrow infiltrative disorders could also present with a disease in the maxillofacial region. Further, detection of congenital anomalies in the maxillofacial region is an indication for a more detailed survey to rule out associated anomalies elsewhere.

The surgeon should be able to identify radiological images, correlate the same with the report and the patient's clinical status, and focus on timely decision making and patient management, thus optimizing the patient's outcome. However, in a dire emergency when a radiologist's opinion is not available, or a delay is expected in obtaining the same, an MFS should be able to interpret radiographs that suggest life-threatening conditions like tension pneumothorax, aspiration bronchopneumonia, foreign body aspiration, or pulmonary edema among others. Imaging can help the MFS in narrowing down on the clinical diagnosis, in staging and evaluating the prognosis of a disease process, or in surgical planning by depicting the exact extent of a lesion after diagnosis is confirmed.

MFS' need to communicate with their radiology counterparts the expectations they have from imaging of a case so that the radiologist can provide the relevant anatomical 
and functional information from the region of interest. Each imaging modality has its own reporting language and the surgeon needs to be well versed with the language used and have a clear concept of principles of image formation, specific to the energy source used in the particular investigation.

\subsection{Role of the Department of Imaging and the Radiologist}

The responsibility of the imaging department is conducting the specified diagnostic imaging investigation, interpretation of the tests or procedures by the radiologist and providing the clinician with accurate reports of the performed investigation as early as possible. Clinical radiologists function as key members of multidisciplinary teams, being an integral part of patient care.

\subsection{Energy Forms Used in Diagnostic Medical Imaging: Understanding the Basics}

Different types of energies are used in diagnostic imaging. These energies are imperceptible to the human eye and when they are passed to the desired location in the body, they interact with the body tissues in different ways. The tissue interactions can reveal information about the tissue, but they need to be interpreted. Access to this information is possible only if it is converted into a visible form on a display system. In essence, the requirements for an imaging investigation are; a reliable and reproducible energy form, a subject (the patient) into whom the energy can be sent, and a format that can convert the invisible image into a visible display.

$\mathrm{X}$-rays and gamma rays are photon energies and members of the electromagnetic spectrum having a wide range of wavelengths and frequencies. X-rays have very small wavelengths being lesser than $1 \AA$ unit and greater than $0.1 \AA$ units. This range of specific frequencies and wavelengths of X-rays give them special properties, gaining popularity in diagnostic imaging.

Gamma rays have shorter wavelengths and higher frequencies than $\mathrm{X}$ rays. They are used in nuclear medicine both for diagnostic and therapeutic purposes.

Conventional radiography, including mammography, fluoroscopy, computed tomography (CT), is a modality that generates images of the ionizing radiation. It works on the same basic principle, which essentially involves passing of an X-ray beam through the body followed by absorption and scattering of a portion of the X-ray energy by the body tissues and transmission of the resultant radiographic pattern formed by differential tissue absorption to a detector for recording or further computer processing.
The popularity of X-rays as an imaging modality is due to its optimum penetration power and photochemical and fluorescent properties. Its fluorescent property is useful in converting invisible images into visible light images, while the photochemical property is responsible for conversion of the visible light image into a permanent visible record called radiograph.

Conventional radiography includes plain radiographs of different body parts like chest, skull, PNS, etc., contrast studies of the gastrointestinal tract like Barium studies, contrast studies of the genitourinary tract like Intravenous urography (IVU), and contrast studies of the vascular system like Arteriography, Phlebography, etc.

Fluoroscopy involves a continuous display of real-time movements of moving structures as radiographic images on the monitor, e.g., act of swallowing, movement of the soft palate, movement of the temporomandibular joint, cardiac pulsations, diaphragmatic movements, etc.

Computed Tomography includes cross-sectional imaging with its modifications like 3D, Volume rendering, Multiplanar reconstruction images, C.T. angiography, etc.

Ultrasound imaging or sonography makes use of highfrequency sound waves that are transmitted from the transducer into the body. The reflection of these waves off the body structures forms the basis of ultrasound image formation. The information necessary for image production is provided by the amplitude of the reflected sound signal and the time taken for it to travel through the body. Commonly ultrasound is used in the imaging of the abdomen, fetus, breast, eye, heart, vessels, and the musculoskeletal system. In recent years, there has been much research into ultrasound of the maxillofacial region, and it has been found to have a range of unique applications, including the evaluation of cervical lymph nodes and the detection of space infections and cystic and solid lesions of the head and neck. In early cases of peripherally located carcinoma of the tongue, ultrasonography may be an initial mode of imaging for evaluation.

Ultrasound imaging is not associated with the same risks as ionizing radiation and is considered to be generally safe if used appropriately. Ultrasound energy does have the potential to produce biological effects on the body and can cause minimal heating of tissues and in certain cases produce tiny gas pockets in the tissues and body fluids known as cavitation.

Magnetic resonance imaging makes use of strong magnetic fields and radiofrequency energy to create images. The signals that produce an MR image originate from the protons present in water and fat molecules in the body. Application of a strong magnetic field by the MRI scanner causes the protons within the body to align themselves. Radiofrequency current is then passed into the patient's body creating a varying magnetic field that causes the protons to flip their spins. Turning off the radiofrequency pulse causes the protons to return to their normal spins, producing radio signals that are measured by receivers in the scanner and used to make digital images of the scanned area of the body. 
Many forms of MRI exist, diffusion and functional MRI being the most common. Diffusion MRI helps in diagnosing disease processes like stroke that restricts diffusion of water molecules across body tissues, while functional MRI is used to visualize the functional activity of the brain. MRI does not use ionizing radiation but since it uses strong magnets, any form of metal implants like artificial joints and heart valves, pacemakers, cochlear implants, metal plates, screws, and rods do pose a hazard.

Thermography makes use of specialized cameras that can detect electromagnetic light energy in infrared wavelengths. Medical thermography produces a topographic heat map, displayed in the form of various color shades, based on the differential skin temperature at various dermatomes bearing resemblance to the visible image of the body. It is not a structural imaging technology providing information on structural changes like other modalities and does not provide a diagnosis, rather only aids in reaching a diagnosis. It is completely safe and painless, does not make use of any form of radiation, and is a unique form of imaging in that it can document pain, thus helping to differentiate malingering from an organic cause for the pain.

\subsection{Basic Radiology for the Nonradiologist: What the MFS should know?}

Plain radiographic film interpretation is an essential skill. The current implementation of electronic health records and digitization of the health care system has made radiographic images available throughout the hospital and an MFS may often have to interpret medical images. So, it is important that they know the basics of medical image interpretation, and this knowledge may prove to be crucial in an acute clinical setting. They should be aware of tissue appearances on radiographs and be capable of making a basic distinction between normal and abnormal.

Understanding the concept of density and contrast is fundamental to interpreting an X-ray image (radiographic image). Different body tissues, including bone, soft tissues, fat, and air, all attenuate the X-ray beam differently. Differential absorption of X-ray photons by the body tissues results in variable energy values of emerging photons producing an invisible image, which is converted into visible form as areas of different gray scales. The contrast on the image is the difference between adjacent densities. These densities range from white to black with varying gray shades in between them. If the densities of two tissues adjacent to each other are greatly different (say a white and a black density tissue), then they are said to have high contrast. If the densities of two tissues adjacent to each other are similar, then they are said to have low contrast. They appear similar in color, differing in their shades of gray. When two body structures of identical density are exactly side by side, the line of demarcation cannot be made out and whole of it appears as one structure.

Consider a chest radiograph. What do you think is the whitest component seen on the radiograph? Most commonly the answers given are heart, bones, below the diaphragm, and so on. Rarely does anyone mention that the " $R$ " marker is the whitest. Similarly, if asked which the darkest component is, the popular answer given is, the lung surrounding the heart, very rarely does one mention that the air surrounding the chest is the darkest. Analyzing as to why the " $R$ " marker is white and the air surrounding is dark, the reasoning is that the chest radiograph is nothing but a representation of the tissues in that region in various gray scales between extreme white and extreme dark, depending upon the variable absorption of X-ray photons in their path through the tissues.

The opacity produced on a radiograph by a specific body tissue is a representation of the actual penetrative ability of $\mathrm{X}$-rays to pass through that tissue and then be incident on the film. Air and fat absorb less radiation, allowing more X-rays to pass through and be incident on the radiograph making their images appear black and pale gray, respectively. Bone and metal as is the " $\mathrm{R}$ " marker made of lead absorbs more radiation and so fewer $\mathrm{X}$-rays are incident on the film making them appear white.

Continuing with the example of chest radiograph, what are branching structures that are seen in the lung fields? Are they arteries, veins, bronchi, lymphatics, or a combination of all these? The common answer given is bronchovascular. The correct answer is arteries and their branches, but not the bronchial tree. The explanation is simple. Air in the bronchi is surrounded by air in the alveoli and so no contrast exists between them and they are hence not seen. On the other hand, blood in the arteries is surrounded by air in the alveoli and hence they stand out. Veins do contain blood but are too less in number and do not significantly contribute to the branching structures. Lymphatics are too small to be detected.

If this basis of X-ray image formation is understood, you can easily interpret any radiographic image provided you have sound knowledge of the relevant anatomy, pathology, and pathophysiology.

\subsection{Imaging Investigations}

\subsubsection{Imaging Investigations Using lonizing Radiation (Photon Energies)}

Electromagnetic radiation has a wide range of wavelengths ranging from being smaller than angstrom units to as large 
as many miles. Smaller wavelengths have greater energy and penetration power and are called photon energy meaning packets of energy and not particle energy. The two energies that are of concern in the present context are X-rays and Gamma rays. Passage of these energies through body tissues either for diagnostic or therapeutic purposes results in a series of events beginning with an incidence of the $\mathrm{X}$-ray photon into tissue followed by sequential changes at molecular and atomic levels. The transformation of energy from the photon results in ionization, namely, in the discharge of an electron and a free radical. Hence, such energies are called ionizing radiation and the process involves breakage of enzyme bonds and formation of new molecules manifesting in the form of various repairable and irreparable biological effects.

The concept of photon energy and its effect on body tissues can be explained using the following example. Consider a hot iron ball being passed through a pile of corn (Fig. 8.1). The temperature of the ball after exiting the corn pile is grossly reduced. It is because the ball, during its transit through the pile, shares the energy with the corn in contact with it. The corn in very close contact to the ball is burnt to ashes, the corn a little farther away, is burnt to charcoal, corn still further away is converted to popcorn, while corn far away from the ball remains unaffected. The effect on the corn is directly proportional to the energy transferred from the hot iron ball to the corn. The same thing happens when photon energy passes through the body tissues with changes occurring at atomic level.

\subsubsection{X-Rays}

$\mathrm{X}$-rays are a form of electromagnetic radiation. Man-made $\mathrm{X}$-rays are produced when fast-moving electrons undergo sudden deceleration on collision and interaction with the target anode in an X-ray tube. The electrons are emitted by the cathode, on being heated up by the electric current flowing through it.

Though conventional X-ray imaging has evolved over the past 100 years, the basic principle remains the same. On passage of an X-ray beam through the body, the beam is attenu- ated by the body's internal structures, being partly absorbed and partly scattered. The remnant of the X-ray energy is transmitted to a detector that records the image for later evaluation. The recording of the radiographic pattern may be on film or other electronic devices. The image created is due to weakening of the $\mathrm{X}$-rays by tissues of varying density encountered along the beam's travel path. Bone being dense tissue, absorbs or attenuates a great deal of the $\mathrm{X}$-rays, while soft tissue being much less dense attenuates or absorbs far less X-ray energy. These differences in absorption help in creating images that can clearly show normal tissue appearance as well as different forms of pathology.

Conventional radiography, fluoroscopy, and CT all work on the same basic principle differing only in their purpose. In radiography, a single image is recorded for later evaluation, in fluoroscopy X-ray images are displayed on a monitor in a continuous flow, thus allowing monitoring of a procedure or contrast passage in real time and in CT imaging a multitude of images are recorded as the detector rotates around the patient's body followed by reconstruction of the individual images as cross-sectional images of internal organs and tissues.

\subsubsection{Computerized Tomography (CT/Spiral CT)}

Computerized axial tomography is a computer-controlled radiological study that produces a reconstructed image of a cross-sectional slice of the desired section of the body. Its features include the absence of superimposition, less scatter and better tissue differentiation of the anatomical slice, thinner slices producing a better reconstructed image. The major drawback of conventional radiography is the overlapping of anatomical structures resulting in difficulty in evaluation of desired structures in a particular plane. The imaging method 'Tomography' was introduced to overcome this drawback and involves imaging of anatomical structures at the desired plane with the blurring of overlapping structures. Classical conventional tomography had its own inherent drawbacks, which included poor-quality blurred images, high radiation dosage, and use of complicated techniques which led to it being replaced by tomography assisted by computer technol-
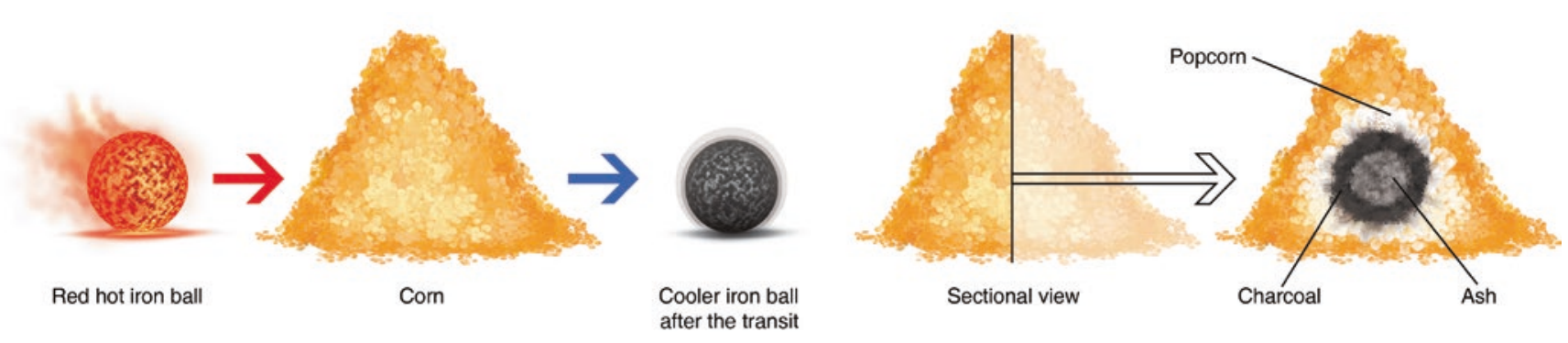

Fig. 8.1 Illustration of passing a hot iron ball through a pile of corn and its effect on the corn 
ogy called 'Computer assisted Tomography' (CAT). With a rise in popularity, CAT has been replaced by the word C.T. and technological advances have resulted in the development of better equipment capable of scanning the entire body within a few seconds. This provides fine anatomical details of submillimetric structures, allows tracing of blood flow in all its phases, and also permits clear depiction of information related to moving structures like the heart in real time as well as their external and internal morphology. Two types of CT have been described based on the shape of the X-ray beam, the traditional fan beam $\mathrm{CT}$ and cone beam $\mathrm{CT}$.

CT Unit Components (Fig. 8.2a, b)

The essential components of a CT unit include:

- The X-ray tube — a specialized tube of high heat loading and dissipating capacity with a very small focal spot, as a source of X-ray energy

- Detectors that receive and quantify the attenuated X-ray photons

- A computer of high capacity that evaluates attenuated photon energy from detectors, stores the values, and converts these values to sectional images

- A console that manipulates all data stored in the computer, helping in reconstructing the image at desired planes with volumetric imaging or multiplanar reconstruction, 3D and volume rendering, followed by projection of the desired structures based on their density values, etc.
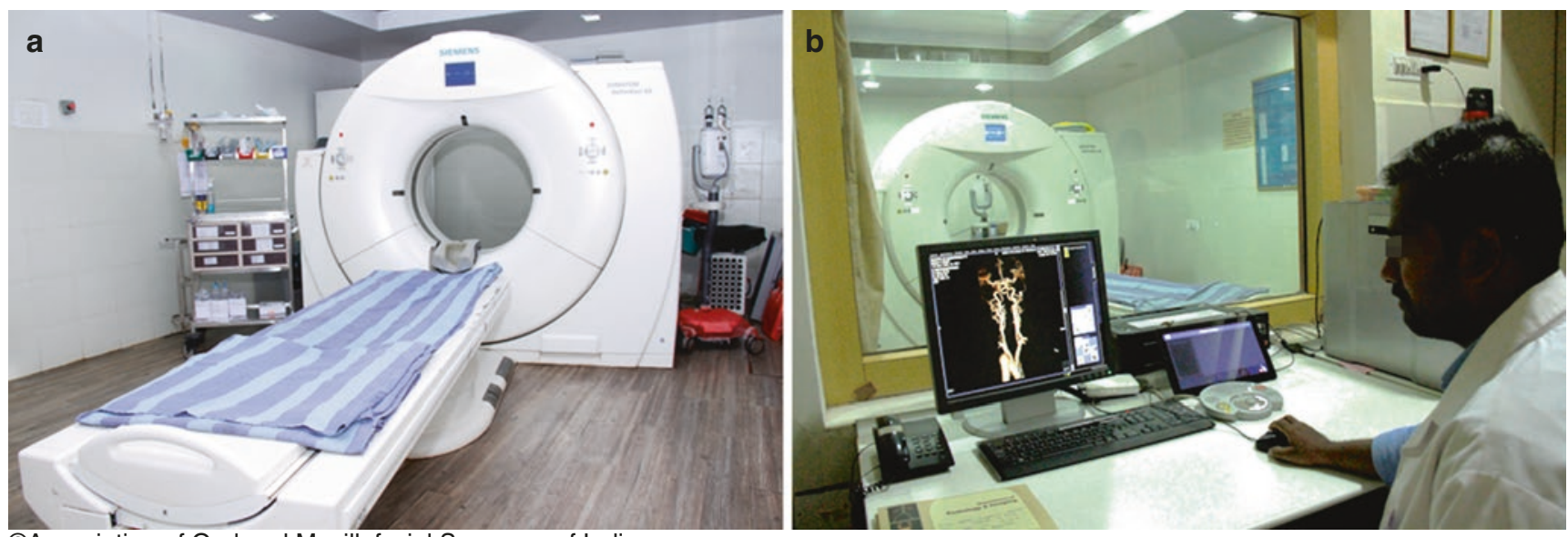

- A gantry that houses X-ray tubes, detectors, and related cables with a central aperture for the patient's body to enter for sectional imaging

- A moving table or a couch that facilitates the movement of the body with extreme accuracy at even submillimetric increment

CT scans have many benefits that far outweigh their risk. It is quick and easy, widely available, and provides superior anatomic and bone details. It has Volume Rendering Technique (VRT), Maximum Intensity Projection (MIP), 3D and Shaded Surface Display (SSD) capabilities, provides multiplanar reconstruction images in desired planes, and is the modality of choice for follow-up studies. (Figs. 8.3a, b, 8.4a, b, 8.5a, b, 8.6a, b, 8.7a, b, 8.8a, b, 8.9a, b, 8.10, 8.11, and 8.12).

The possible risks of using CT imaging include exposure to ionizing radiation and the predicament of suffering an allergic reaction due to use of contrast media. One drawback of CT when compared to MRI is the lesser resolution of softtissue detail. Other disadvantages are the associated cost and the limitations of its use in the pediatric age group due to the need of sedation.

\section{CT Limitations}

Motion and beam-hardening artifacts used to be considered as major drawbacks, but they have been markedly minimized with advances in technology. A physical limitation is that the patient needs to weigh less than 300 to $400 \mathrm{lbs}$. For

CAssociation of Oral and Maxillofacial Surgeons of India

Fig. 8.2 (a) CT room shows Gantry, Patient couch, Emergency crash trolley, Central suction, and Oxygen and Pressure Injector. (b) Control room showing a CT technician working with various controls and the image acquisition system 



CAssociation of Oral and Maxillofacial Surgeons of India

Fig. 8.3 (a) Museum Model of head showing how axial slices are planned. The slice thickness varies from submillimeter to $10 \mathrm{~mm}$, depending on the ROI (region of interest) and anatomical structure to be seen. (b) Stacked CT slices showing specific anatomy at a specific slice

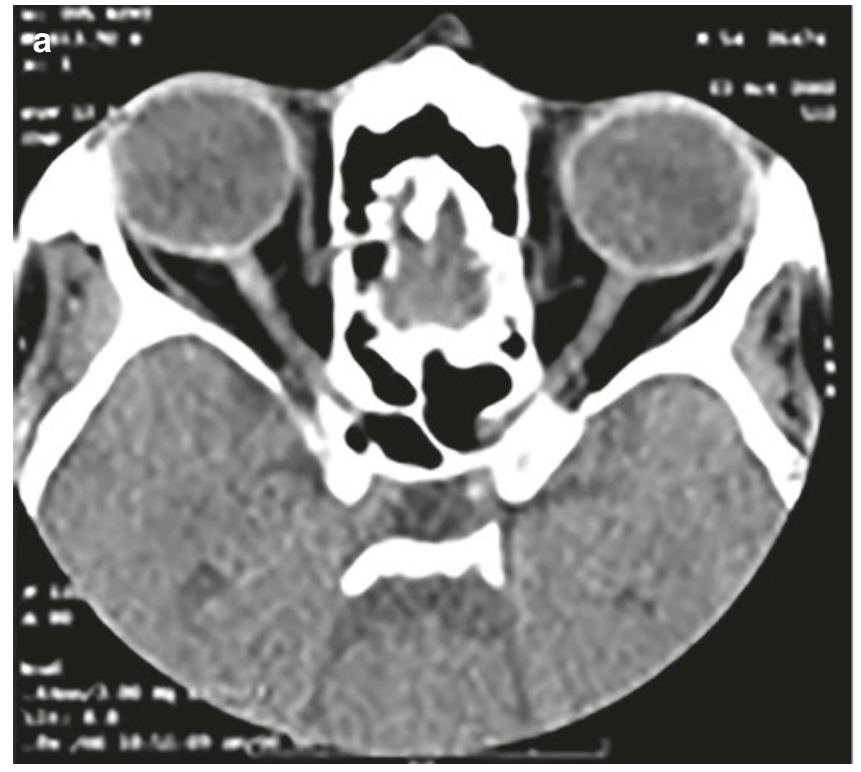

CAssociation of Oral and Maxillofacial Surgeons of India

Fig. 8.4 (a) CT axial primary slice soft-tissue window at the level of optic nerves showing mediolateral and anteroposterior relations and not supero inferior relations. (b) CT sagittal reconstructed image of the

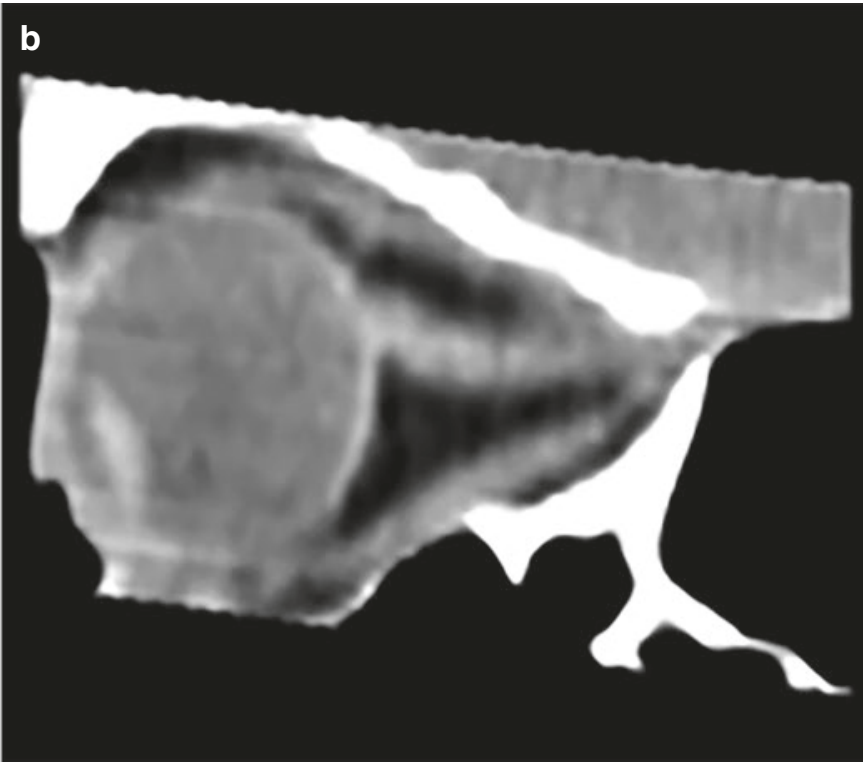

same patient at the level of optic nerve showing anterior, posterior, superior, and inferior relations 

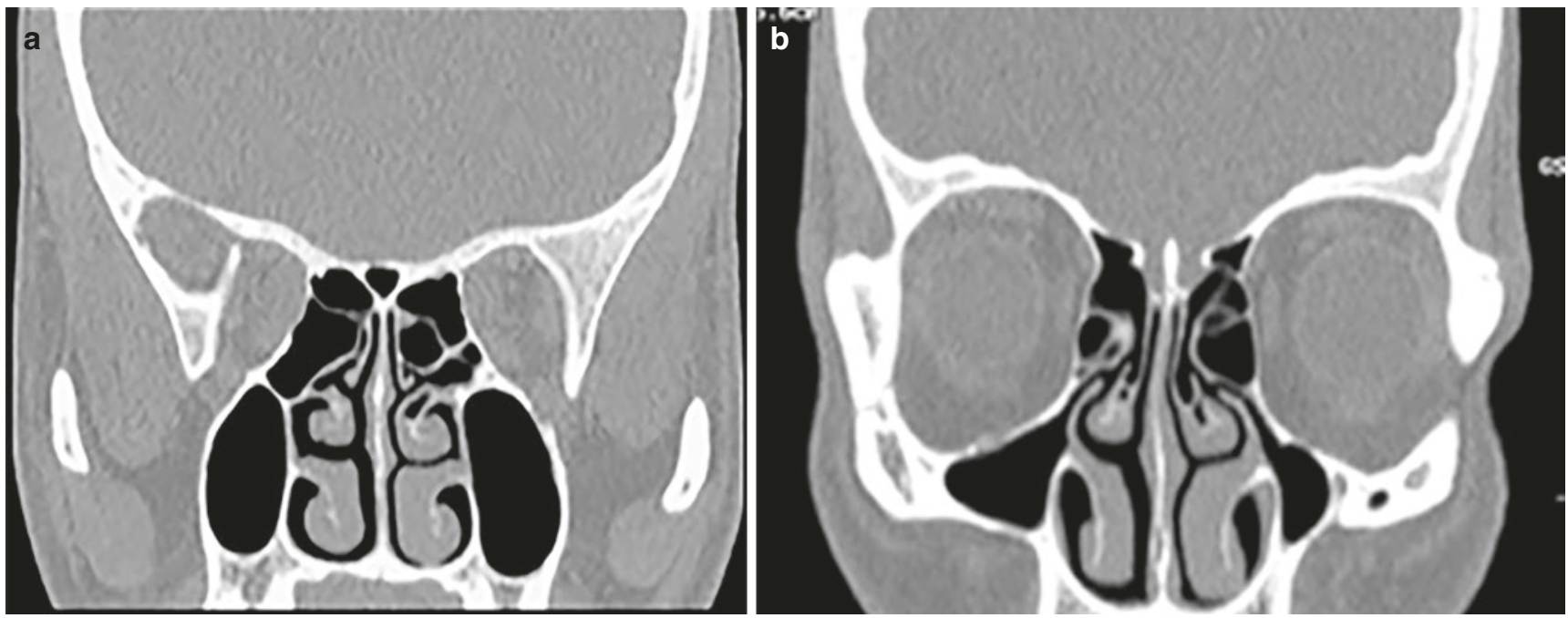

CAssociation of Oral and Maxillofacial Surgeons of India

Fig. 8.5 (a) and (b).CT coronal bone window primary images at different levels of paranasal sinuses


CAssociation of Oral and Maxillofacial Surgeons of India

Fig. 8.6 (a) and (b).CT coronal soft-tissue window primary images at different levels of Paranasal sinuses 

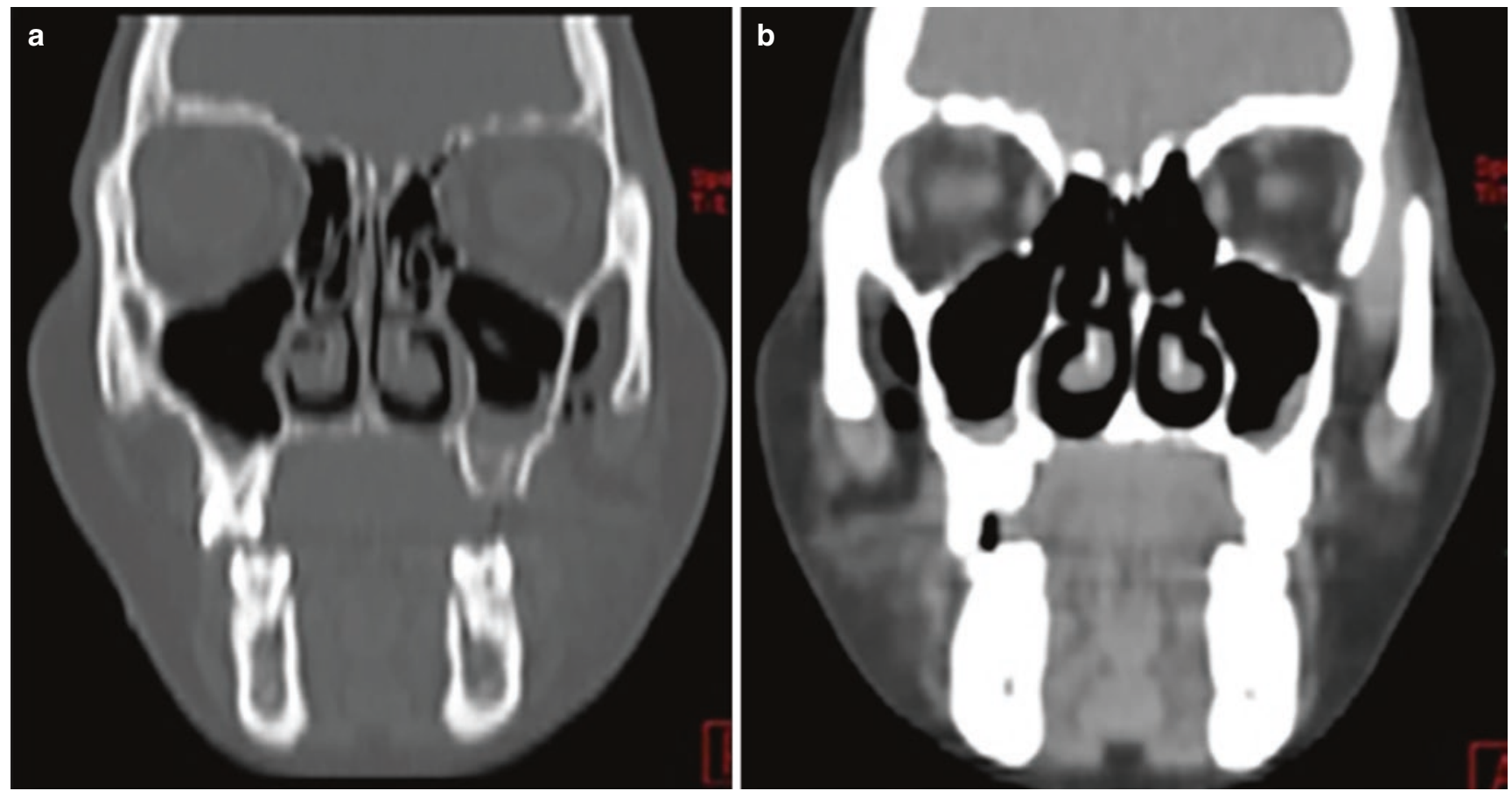

CAssociation of Oral and Maxillofacial Surgeons of India

Fig. 8.7 (a) and (b). CT reconstructed coronal images from thicker slices of paranasal sinuses at different levels. (a) bone window. (b) soft-tissue window. Note: coarse appearance
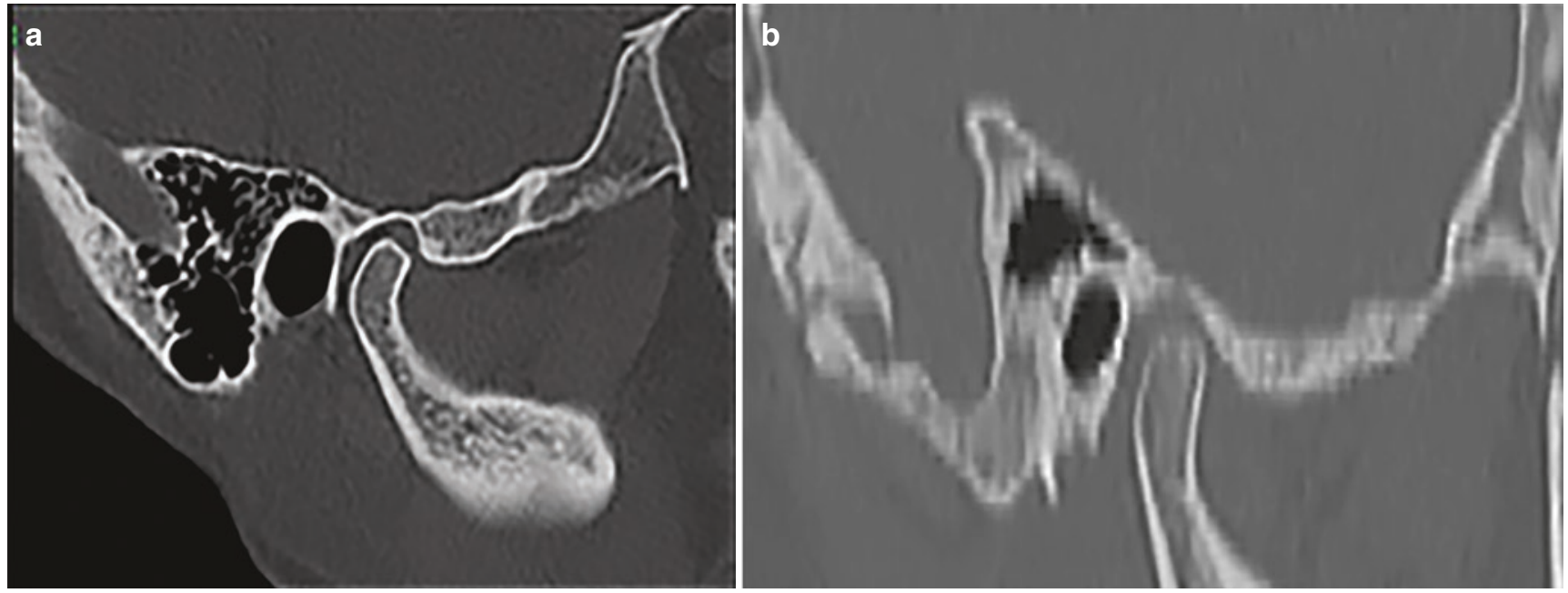

CAssociation of Oral and Maxillofacial Surgeons of India

Fig. 8.8 (a) CT direct slice of T.M. joint in its sagittal plane. Bone window. (b) CT reconstructed image from primary axial thick slices of T.M. joint in its sagittal plane. Note: coarse appearance 



CAssociation of Oral and Maxillofacial Surgeons of India

Fig. 8.9 (a) CT 3D reconstructed image for surface bony details from thicker slices. Note: coarse appearance. (b) CT 3D reconstructed image from thin slices. Note: better and smoother image



CAssociation of Oral and Maxillofacial Surgeons of India

Fig. 8.10 CT 3D surface rendering reconstructed image for surface soft-tissue details from thinner slices

CT imaging of the head region, axial and direct coronal slices are primarily possible, for the neck region, only axial slices are possible and in the temporomandibular joint region axial and direct sagittal slices are possible. It is through the raw data thus available that images are reconstructed in any desired plane.

\section{Dentascan}

Basic principles of CT Orthopantomography $(O P G)$ and Dentascan

Conventional OPG is a first-level diagnostic exam that provides a gross panoramic view and just enough information about alignment of the teeth and any other related gross pathology. Unavoidable overlapping of some of the anatomical structures hinders accurate evaluation. To overcome these drawbacks, Dentascans and CT OPGs are used. They are extended applications of CT scan and involve the acquisition of thin axial submillimeter slices of the region covering the upper and lower jaws (Fig. 8.13). Reconstructed images obtained using these raw data image slices form the CT OPG or Dentascan image (Fig. 8.14a, b).

Coronal reconstructed images form the C.T. OPG (Fig. 8.15a, b, c). It is a second-level imaging technique wherein evaluation of the osseous elements of the maxilla and mandible is far better in comparison to conventional OPG. It provides the MFS with a better understanding of the morphology of the roots and the degree of contiguity between the roots and the mandibular canal. The information acquired is then used in planning and performing minimally invasive surgical procedures [1]. 

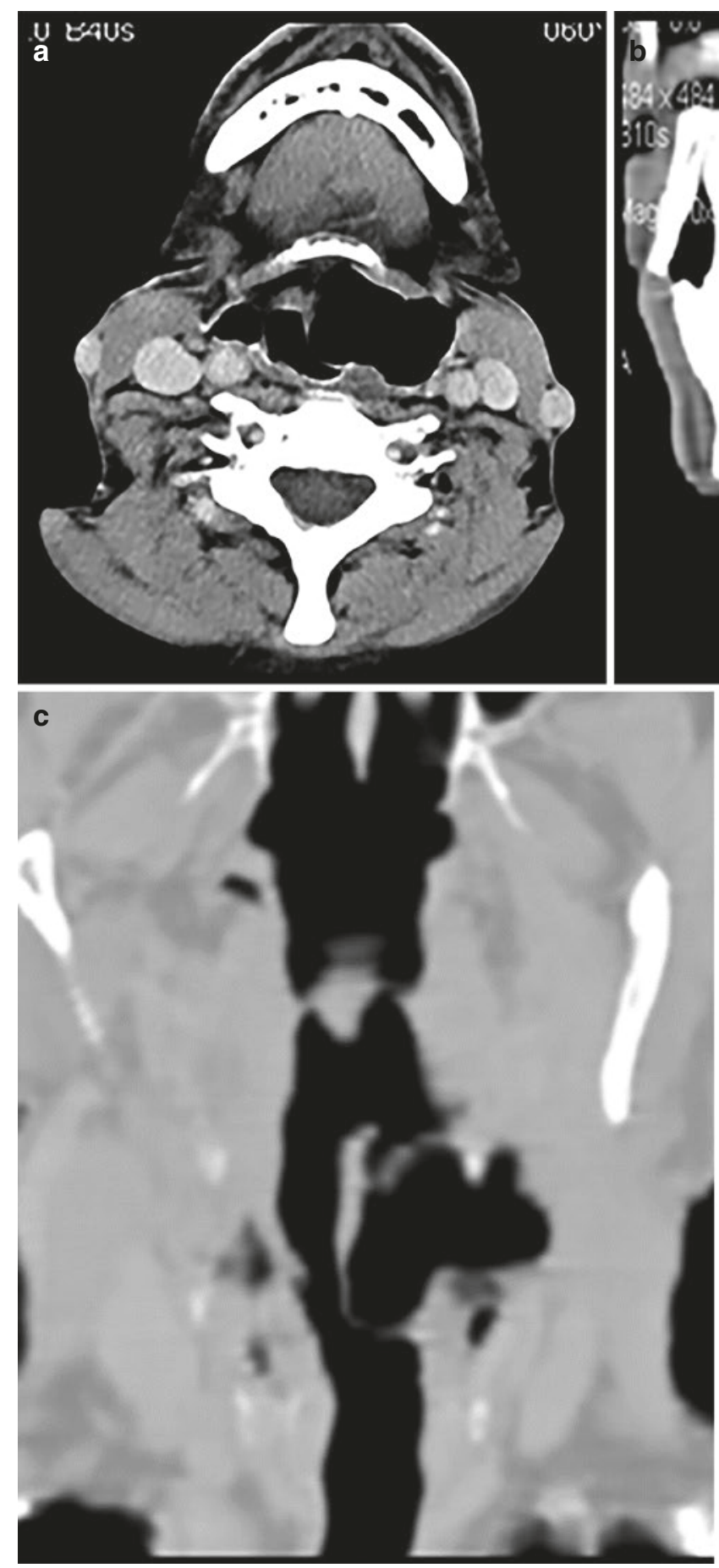

(CAssociation of Oral and Maxillofacial Surgeons of India



d



Fig. 8.11 (a) CT Primary axial slice of neck at midlaryngeal level. (b) and (c) Sagittal and coronal reconstructed images from sequential CT primary axial slices. (d) CT Volume rendering technique (VRT) image of air column in the pharynx, larynx, and tracheal region as minimum density projection from primary axial slices (a). Note: Better visualization of laryngocele 




(CAssociation of Oral and Maxillofacial Surgeons of India

Fig. 8.12 CT angiogram: 3D VRT (Volume Rendering Technique) displaying carotid arteries and its branches along with carotid body tumor. It is a reconstructed image from primary thin axial CT slices

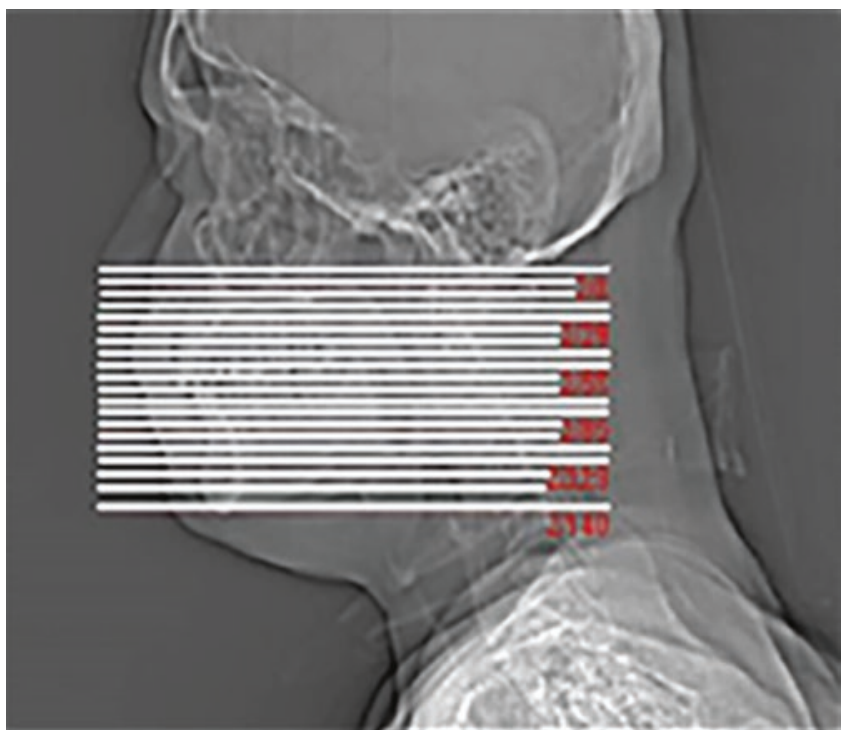

CAssociation of Oral and Maxillofacial Surgeons of India

Fig. 8.13 CT Scanogram for dentascan showing submillimeter axial slices planning in the region of maxilla and mandible


CAssociation of Oral and Maxillofacial Surgeons of India

Fig. 8.14 (a) and (b) .Reformatted panoramic CT images (b) are procured at desired plane of the jaw by curvilinear graphic lines drawn on the primary axial slice (a) selected 

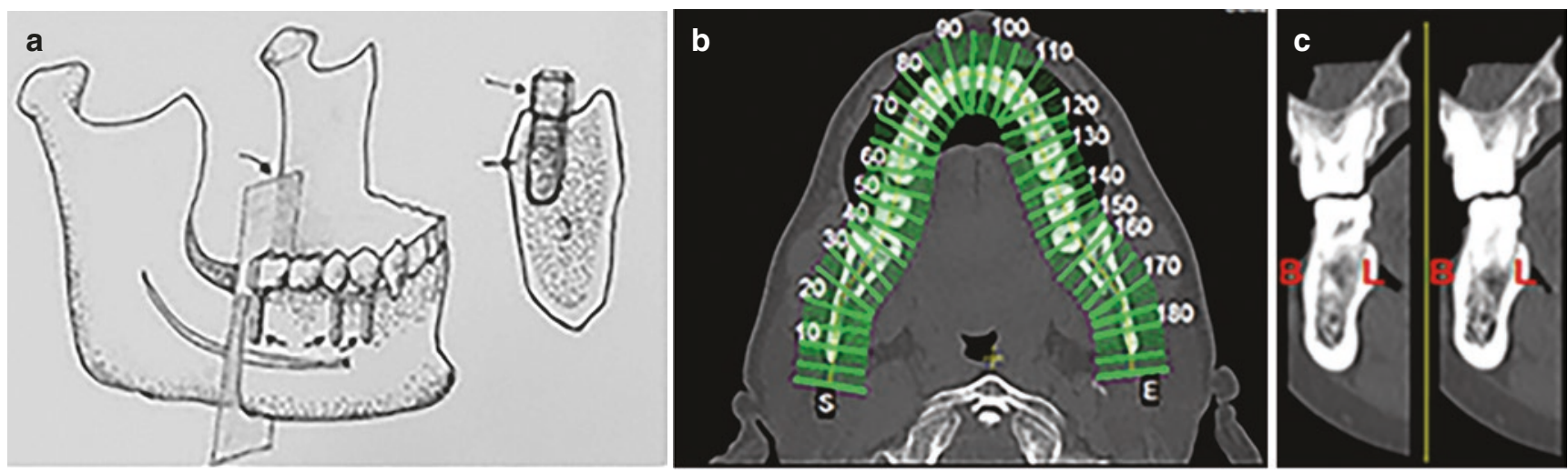

CAssociation of Oral and Maxillofacial Surgeons of India

Fig. 8.15 (a), (b), and (c). a and b show coronal plain image planning of individual tooth. c shows the coronal reformatted image of tooth

a

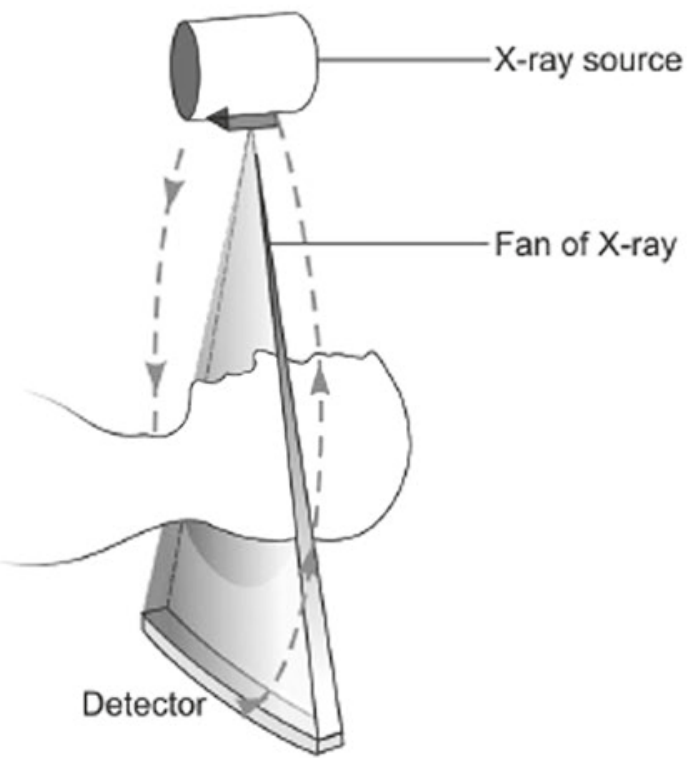

b

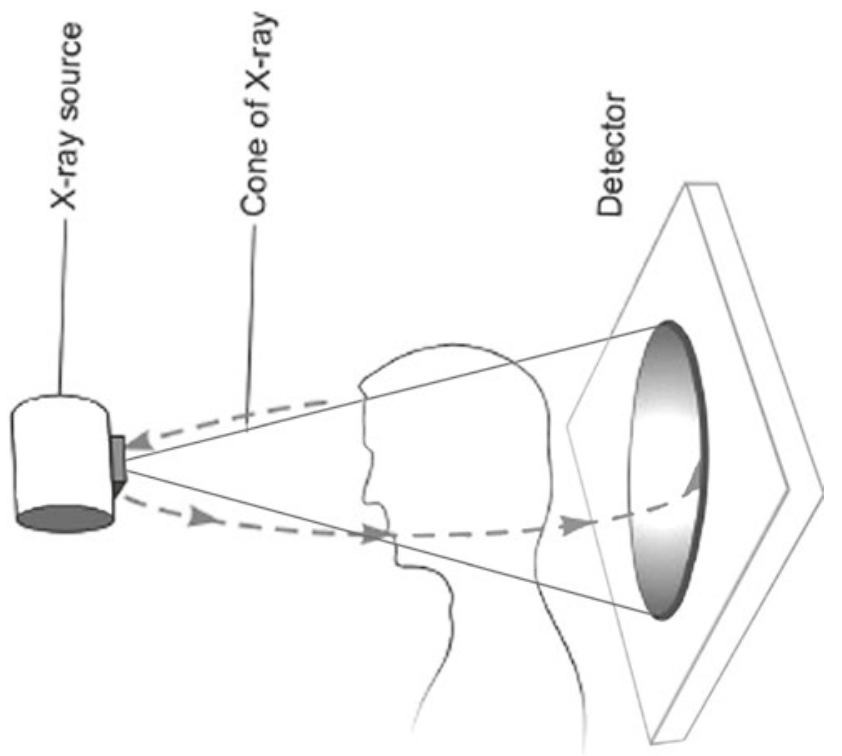

(CAssociation of Oral and Maxillofacial Surgeons of India

Fig. 8.16 (a) and (b). Schematic diagram. (a) shows fan beam of X-rays from X-ray tube with curvilinear row of detectors. (b) shows cone beam of X-rays from X-ray tube with flat detector

\section{Cone Beam CT (CBCT)}

\section{Indications for $C B C T$}

CBCT applications have proved helpful in a multitude of dental disciplines and the MFS needs to be aware of it. Its indications include periodontal and endodontic assessment, placement and evaluation of implants, evaluation of jaw bones, temporomandibular joints, bony and soft-tissue lesions, and alveolar ridge resorption and orthodontic evaluation.

\section{Working Principle and System Composition of CBCT System}

As the name suggests, it is a form of computed tomography (CT). The basic process involved in CBCT is the scanning of the region of interest using a cone-shaped X-ray beam, making a single rotation around the vertical axis of the patient's head. The information obtained, from various angles about the structures in the region of interest, is in a digitized format relating to shape and density, which is then processed using 
Table 8.1 Comparison between CBCT and Fan Beam CT

\begin{tabular}{|c|c|}
\hline Fan Beam CT & CBCT \\
\hline $\begin{array}{l}\text { Conventional CT scanners } \\
\text { make use of a fan beam and } \\
\text { provide a set of consecutive } \\
\text { slices of image. }\end{array}$ & $\begin{array}{l}\text { CBCT makes use of a cone beam, } \\
\text { which radiates from the x-ray source } \\
\text { in a cone shape, encompassing a } \\
\text { large volume with a single rotation. }\end{array}$ \\
\hline $\begin{array}{l}\text { Makes use of a lie-down } \\
\text { machine with a large gantry }\end{array}$ & $\begin{array}{l}\text { Makes use of a sitting up machine of } \\
\text { smaller dimensions }\end{array}$ \\
\hline $\begin{array}{l}\text { Greater contrast and } \\
\text { resolution }\end{array}$ & Ease of operation \\
\hline $\begin{array}{l}\text { Better differentiation between } \\
\text { tissue types (bone, teeth, and } \\
\text { soft tissue) }\end{array}$ & $\begin{array}{l}\text { Commonly used for hard tissues } \\
\text { Especially dental tissues }\end{array}$ \\
\hline Higher radiation dose & Lesser radiation dose \\
\hline Detector type $\mathrm{X}$-axis only & Detector type X-and Y-axes \\
\hline $\begin{array}{l}\text { Volume acquisition } \\
\text { requirements are sequential } \\
\text { multiple gantry rotations }\end{array}$ & $\begin{array}{l}\text { Volume acquisition requirements are } \\
\text { single gantry rotations }\end{array}$ \\
\hline Speed of examination is fast & Speed of examination is faster \\
\hline
\end{tabular}

special software and a three-dimensional (3D) image is reconstructed [2].

CBCT occupies a special place in imaging of the Head and Neck region. It offers a number of advantages over traditional CT technology. It has a multitude of uses in the dental discipline, including dentoalveolar disease and anomalies, jaw tumors, vertical root and dentin fractures, among others. It has also had a positive impact on teaching students about oral and maxillofacial imaging making them adept at operating equipment and related instruments, resulting in greater accuracy in evaluation and analysis of the pathological characteristics of the disease process leading to a proper final clinical diagnosis. Both the traditional fan beam CT and CBCT are third-generation CT scanners but are different in some ways (Fig. 8.16a, b) (Table 8.1).

\subsubsection{Gamma Rays}

Gamma rays are a form of Electromagnetic radiation (EMR). They are similar to X-rays but can be differentiated by the fact that they originate from the nucleus. Gamma-ray photons have the highest energy with their waves having the shortest wavelength in the EMR spectrum. The difference between the two is that X-rays are produced by accelerating electrons when they strike a target, while gamma rays originate from the nucleus of a radionuclide after radioactive decay. Gamma rays damage the DNA of cells and it is this action that is used in the treatment of cancerous tumors, care being taken not to affect the DNA of the surrounding healthy tissue cells [3].

\subsubsection{Nuclear Isotope Studies}

Scintigraphy or radionuclide imaging is a modality that is readily available and relatively inexpensive. One drawback is that though it can provide specific information, the resolution is poor. It is a functional imaging modality and can assess early physiological changes even before bone mineral changes are evident. Bone scintigraphy is highly sensitive in detection of skeletal osteoblastic activity and the process involves uptake of the radiopharmaceutical Technetium-99 m (Tc-99 m), by the mineral component of the bone due to its affinity for bone. It has proven to be greatly useful in the study of malignant lesions and also in the evaluation of vascularized bone grafts used in reconstruction surgeries of the maxillofacial region [4]. Another use of radionuclide imaging is in evaluating patients with condylar hyperplasia. In these patients, radionuclide imaging of the temporomandibular joint is performed to exclude active condylar growth and in planning the extent of orthognathic surgery. A progressive condition necessitates the removal of the entire condyle and neck while trimming of the enlarged condyle suffices otherwise [5].

\subsubsection{Positron Emission Tomography (PET)}

PET also is a functional imaging technique. It is unique in that it produces images reflecting in vivo changes in tissue metabolism, linking the changes to malignancy. Body images are produced when radiation is emitted from radioactive tracer substances like Carbon-11, Fluorine-18, Oxygen-15, or Nitrogen-13. When a positron emitted from the radioactive substance collides with an electron in the tissue, gamma rays are given off at the site, which are detected by a gamma detector. FDG-PET/CT fusion scans of the head and neck are not primarily for infection identification but for categorizing a clinically questionable lesion as malignant or not.

CT scans detect changes in body structure and only confirms the presence of a mass. PET scan is different from CT in that it can reveal the presence of disease earlier allowing for quicker diagnosis, can detect the extent of disease and whether a detected mass is benign or malignant, helps monitoring treatment and its effectiveness, and can detect abnormalities even before there is any anatomical change. This is based on the fact that glucose consumption by malignant 
cells and hence tissue is far higher than that of normal tissue.

The scan involves intravenous injection of a radiolabeled compound like 2-deoxy-2-(18F) fluoro-D-glucose (FDG). After uptake of the compound, it undergoes further break down in the cells. Since tumor cells have a high metabolic rate, it is also metabolized by the tumor cells. FDG is metabolized to FDG-6-phosphate, but the tumor cells are incapable of metabolizing it further and in consequence the FDG-6phosphate accumulates and concentrates within the tumor cells. It is this accumulation that is detected and quantified. As compared to normal tissue, the uptake of FDG by malignant tissue is very high, making it a sensitive method to pick up early malignant tissue [6].

\subsection{Radiation: Hazard Awareness and Principles of Protection}

\section{Hazardous Nature of X-Rays}

When an X-ray photon is incident on tissue, it results in a series of changes at the molecular and atomic level. The transformed photon energy results in the discharge of an electron and a free radical, or ionization in other words. Ionization results in the production of a new molecule due to breakage of enzyme bonds manifesting in the form of different biological effects. These effects may be repairable when there is no appreciable damage, or irreparable when there is definite occurrence of damage, the adverse effect being cumulative in nature.

Myths and Facts about radiation in the field of Diagnosis: Many myths exist regarding radiation hazards associated with medical diagnostic imaging. Possible reasons for this are the available historical data, exaggerated social media reports, results of experimental studies and the complex nature of radiation biology making it difficult to evaluate its mechanism of action. Historical data relates to the reports of visible radiation hazards from studies on victims of the Hiroshima/Nagasaki bomb explosion in 1945 who were followed up for 15-18years. Exaggerated reporting by social media about nuclear reactor accidents like that at Chernobyl add to the existing myths. The extremely short time for which radiation interacts with matter $\left(10^{-5} \mathrm{Sec}\right)$ after absorption, makes it difficult to study the complex nature of radiation biology. The biological process of cell death can take days to months, carcinogenesis can take years to occur while generations may pass before a mutational change is seen.

In reality, the dose of received radiation from man-made $\mathrm{X}$-rays that exist today is minute, in comparison with the massive radiation exposure that occurred in the aforementioned examples. It is true that in the infant stage following discovery of X-rays and its use in diagnosis, visible radiation hazards did occur, which was due to ignorance in the understanding of its potential hazards and the use of crude equipment resulting in high amounts of radiation leak. With passage of time, we have learnt to put the benefits of X-rays to full use, concurrently taking proper precautions to protect ourselves from its hazardous effects. Over the years, improvements in diagnostic equipment using ionizing radiation have led to the production of newer equipment providing the best possible image quality with minimal radiation exposure and improved devices that are protective against radiation. Yet, the fact remains that the best method to reduce the effects of radiation is using it only when it is an utmost necessity [7].

\subsection{Imaging Investigations Not Involving lonizing Radiation}

\subsubsection{MRI (Magnetic Energy Coupled with Radiofrequency Energy)}

This imaging modality does not involve the use of ionizing radiation but makes use of magnetic and radiofrequency energy to obtain information of the internal organs. Components of an MRI unit include the magnet, the console, and the computer.

\section{Image Procurement by MRI}

Exploiting the body's magnetic property forms the basis of imaging using magnetic resonance. The human body is abundant in atoms with odd number of protons, or in other words ionized particles or charged atoms. A charged particle spinning around its own axis creates a magnetic field around 
itself behaving like a tiny bar magnet. Hydrogen represents one such atom that is present in water molecules and therefore in all body tissues.

The random orientation of the hydrogen atoms cancels out their magnetic fields and so there is no net magnetization at rest. When these protons are placed in a strong magnetic field, a net magnetization is produced that aligns parallel with the main magnetic field. To influence the human body, the external magnetic strength needs to be about 6000 to 80,000 times the earth's magnetic strength. The resultant net magnetization acts as the source of the MR signal and is used to produce MR images. Application of a radiofrequency (RF) pulse results in absorption of energy from the RF pulse causing the net magnetization to rotate away from the direction of the main magnetic field, the amount of rotation or flip angle depending on the strength and duration of the RF pulse. The strength and duration of the RF pulse can be controlled such that the net magnetization can be rotated to any angle [8]. Withdrawal of the RF pulse results in the liberation of energy, with the emission of signals based on the density of protons or hydrogen atoms. These signals are captured by the external antennae, fed into the computer, and processed to produce the image.

\section{Types of Magnets: Their Advantages and Limitations}

- External magnets are of two types, the permanent magnet and the electromagnet.

- Permanent magnets are cheaper with no significant recurring cost. However, its limitations include limited strength of less than 0.5 tesla, heavy nature making transportation difficult, production of weak signals, and slower performance with limited range resolution.

- Electromagnets work by the passage of high current around ferromagnetic substances creating the required magnetic field. They are faster, have better resolution, produce better reconstructions, and are lighter than permanent magnets. The drawback is the production of large amount of heat that needs cooling using Liquid Helium. Other disadvantages include the high cost of infrastructure, high consumption of electricity, and recurring expenses.

\section{Preparing a Patient for an MRI}

- Detailed history and clinical examination are mandatory.

- Specific history relating to previous surgery with the use of aneurysmal clips or metallic prosthesis needs to be ascertained.

- Patient compatibility for the procedure needs to be evaluated in view of the requirement of lying motionless for a minimum of 30 to $40 \mathrm{~min}$, e.g., Orthopneic patients, COAD patients. Anesthesia might be required for agitated patients and infants.
- A double check for any metallic objects on the patient's body using a metal detector device is necessary.

- Claustrophobic patients need to be reassured and put at ease before the examination begins.

- The procedure needs to be explained in detail prior to the patient entering the MRI magnet room.

CT \& MRI are complementary to each other. As far as the MFS is concerned, thin-slice CT with reconstruction is required while evaluating bony lesions, especially in cases of trauma. MRI on the other hand is good for evaluation of softtissue tumors and is contraindicated in penetrating injuries of the eye (Table 8.2).

Table 8.2 CT and MRI: A comparison

\begin{tabular}{|c|c|c|}
\hline & CT & MRI \\
\hline $\begin{array}{l}\text { Multiplanar } \\
\text { Imaging }\end{array}$ & $\begin{array}{l}\text { Only axial slices are } \\
\text { possible. } \\
\text { Images need to be } \\
\text { reconstructed in } \\
\text { multiple planes } \\
\text { following acquisition } \\
\text { of primary axial } \\
\text { sections. Direct } \\
\text { sagittal and coronal } \\
\text { slices are possible } \\
\text { except for TM joint. }\end{array}$ & $\begin{array}{l}\text { Direct multiplanar } \\
\text { images can be primarily } \\
\text { acquired in any desired } \\
\text { plane. } \\
\text { Excellent sectional } \\
\text { images are possible. }\end{array}$ \\
\hline $\begin{array}{l}\text { Soft-tissue } \\
\text { characterization }\end{array}$ & & Best with MRI than CT \\
\hline $\begin{array}{l}\text { Compact bone/ } \\
\text { calcified lesions }\end{array}$ & $\begin{array}{l}\text { Best analyzed by CT } \\
\text { than MRI }\end{array}$ & \\
\hline Bone marrow & & $\begin{array}{l}\text { Assessment best with } \\
\text { MRI than CT }\end{array}$ \\
\hline Radiation & $\begin{array}{l}\text { Ionizing radiation } \\
\text { involved }\end{array}$ & $\begin{array}{l}\text { No ionizing radiation } \\
\text { involved }\end{array}$ \\
\hline Motion artifacts & $\begin{array}{l}\text { Relatively well } \\
\text { managed with CT }\end{array}$ & $\begin{array}{l}\text { Very sensitive to any } \\
\text { kind of motion. May } \\
\text { require anesthesia. } \\
\text { Noisy breathing too may } \\
\text { affect images }\end{array}$ \\
\hline Time required & $\begin{array}{l}\text { Faster, with whole } \\
\text { body scan from vertex } \\
\text { to toe taking } \\
8-10 \text { seconds }\end{array}$ & $\begin{array}{l}\text { Takes longer. Minimum } \\
\text { time required for scan of } \\
\text { any part is } 40-60 \mathrm{~min}\end{array}$ \\
\hline Contrast media & $\begin{array}{l}\text { Invariably required } \\
\text { and so contrast-related } \\
\text { problems are } \\
\text { unavoidable. }\end{array}$ & $\begin{array}{l}\text { Contrast is less } \\
\text { frequently used and is } \\
\text { relatively safer. }\end{array}$ \\
\hline Trauma & Best modality & \\
\hline $\begin{array}{l}\text { Ferromagnetic } \\
\text { artifacts }\end{array}$ & & $\begin{array}{l}\text { MRI is contraindicated in } \\
\text { patients with pacemakers } \\
\text { or recently placed } \\
\text { implants. MRI } \\
\text { compatible supportive } \\
\text { gadgets: Pulse Oxymeter, } \\
\text { anesthesia equipment, } \\
\text { I.V. stands, etc. }\end{array}$ \\
\hline
\end{tabular}




\section{How Does CT/MRI Differ from Conventional Radiography?}

CT and MRI are sectional imaging methods, in which the whole body or the region of interest is sectioned into serial slices and further into cubes (Fig. 8.3a, b). Each slice surface represents the corresponding sectional anatomy with each slice being made up of small units or cubes with each cube representing the basic nature of the tissue in the digital form (Figs. 8.17 and 8.18a, b). Different modalities produce varied imaging appearances of the same structure. For instance, if a coconut is imaged using different modalities, the fine structural details of the interior of the tender coconut, and its contents are better seen on CT and MRI (Fig. 8.19a-c). Similarly, imaging of a pineapple in axial (Fig. 8.20a-d) and coronal planes (Fig. 8.21a-c) and using the volume-rendering technique (Fig. 8.22) reveals varied appearances on CT and different MRI sequences.

To summarize, in sectional imaging, the region of interest or the whole body is divided into slices and cubes with each cube representing the basic tissue of that region with thinner slices and smaller-sized cubes offering better resolution.



CAssociation of Oral and Maxillofacial Surgeons of India

Fig. 8.17 Each axial slice is divided into small cubes loaded with digitalized values representing corresponding tissue volume called voxel. Its surface represents a fraction of the grayscale image specific for that region called pixel. Thinner the slice and smaller the pixel, better is the resolution


CAssociation of Oral and Maxillofacial Surgeons of India

Fig. 8.18 (a) and (b). Figures showing unit wise volumetric digitalized information of anatomical structures for retrieval and displayed as sectional anatomical slices 

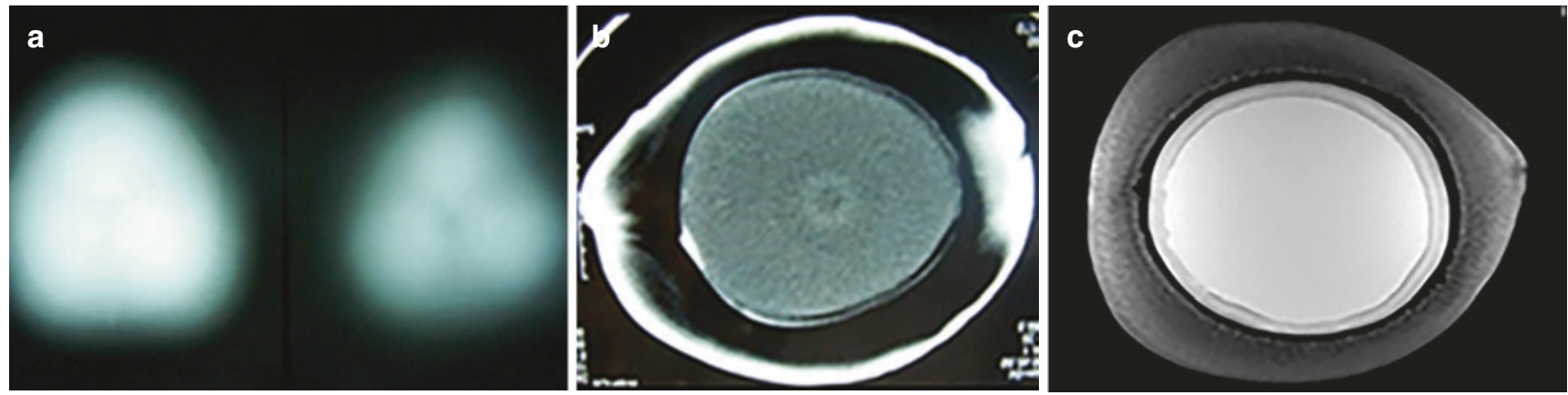

CAssociation of Oral and Maxillofacial Surgeons of India

Fig. 8.19 (a), (b), and (c). Imaging of tender coconut. (a) Conventional plain Radiograph. (b) CT Axial sectional imaging. (c) MRI sectional imaging. Note: added information with each of these modalities


CAssociation of Oral and Maxillofacial Surgeons of India

Fig. 8.20 (a), (b), (c), and (d). Axial sectional images of pineapple taken at the same level (a) CT. (b, c, d) MRI various sequences. Note: superior soft-tissue resolution in MRI, highlighting various components of soft tissue in various sequences 

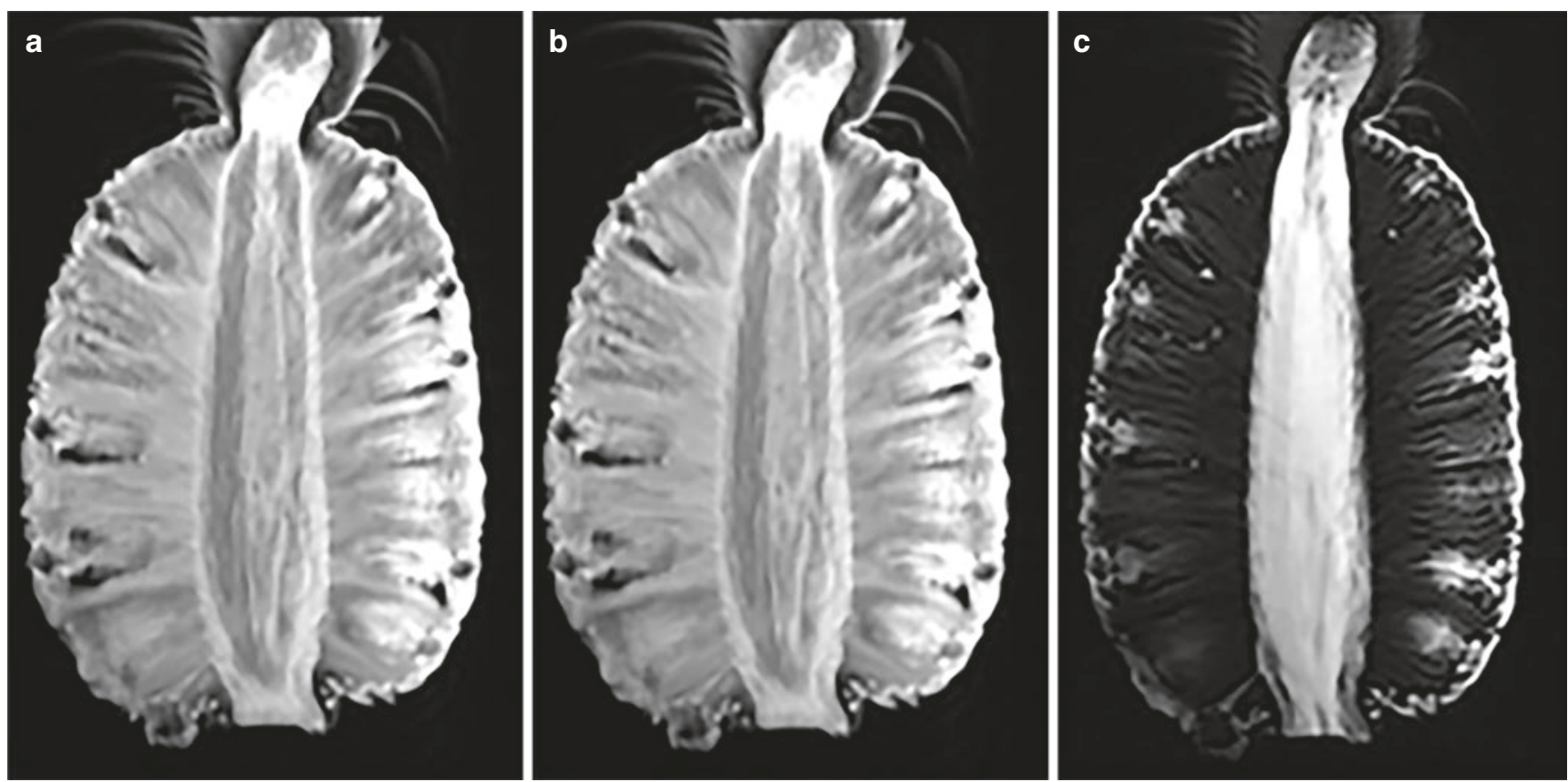

CAssociation of Oral and Maxillofacial Surgeons of India

Fig. 8.21 (a, $\mathbf{b}$ and $\mathbf{c})$. Various sequences of MRI of a pineapple showing multiplanar capability to procure sections in any desired plane without reconstruction unlike CT



CAssociation of Oral and Maxillofacial Surgeons of India

\subsubsection{Ultrasound}

It seems implausible that sound waves can produce informative images of internal organs of a human body. Yet it is true that technology has evolved so much over the years that it is now possible to obtain information of body structures using sound waves by making use of the echo and Doppler properties of sound waves.

Sound waves can be classified, based on their wavelength and frequency as audible sounds (20 to 2000 cycles/sec), infrasound (less than 20 cycles/sec), and ultrasound $(>20,000$ cycles/sec). Ultrasound in the range of 2 to $20 \mathrm{MHz}$ is used in medical imaging [8]. The source of these ultrasound waves is piezoelectric crystals. These crystals have the special property of generating electrical pulses on being subjected to pressure. In addition, the passage of electricity through the crystals causes them to get compressed or deformed. Essentially, these crystals are unique in that they have the dual property of converting electrical energy into mechanical energy and mechanical energy into electrical energy. Intermittent passage of electrical current through the crystals makes them alternate between compression and relaxation, resulting in vibration of the crystal, in consequence producing sound energy of small wavelength in the ultrasound range.

Devices, equipped with piezoelectric crystals, having the capability to convert electrical energy to mechanical energy and finally to sound energy as well as having the reverse capabilities of converting sound energy to electrical energy, are termed as Transducers.

Sound waves are useful in imaging due to their property of echo/reflection and due to the Doppler effect. Echo property

Fig. 8.22 CT 3D surface rendering image of Pineapple 
refers to the property of sound waves to get reflected at the interface of two media, the strength of the reflected sound echo depending on the density difference of the media through which they traverse. Reflected sound energy gets converted to mechanical energy and finally to electrical energy on striking the crystal, and this electrical energy is represented on a monitor as bright dots of variable gray scales.

Varying density interfaces throughout body tissues, produces variable image brightness with gray scales ranging between extreme bright dots to black dots, representing different organ or tissue sections. The Doppler effect of sound refers to the change in the apparent frequency of a wave when the observer and the source move toward or away from each other. Motion causes a change in pitch with a higher frequency being heard when the sound source approaches the observer, and a lower frequency being heard when the sound source moves away. This Doppler property is used in evaluating the direction and velocity of flow of blood in vessels. The transducer is considered as the fixed source of sound and the blood component, mainly the RBCs act as moving reflecting bodies. At a given point in the vessel by calculating the sudden change in the frequency of reflected sound, we can calculate the velocity of blood and also the direction of blood flow. This helps in the evaluation of percentage of stenosis in a vessel.

\section{B Mode}

A grayscale sectional image has specific echo texture based on tissue content with the display mode being called as the ' $\mathrm{B}$ ' mode (Brightness mode) or grayscale mode. Higher sound frequency provides better image resolution with delineation of finer tissue components, however, at the expense of depth of penetration. Higher-frequency transducers $(7 \mathrm{MHz}$ to $20 \mathrm{MHz}$ ) are hence used for imaging superficial structures like cheek, the maxillofacial region, neck spaces, breast, thyroid, testis, eye, musculoskeletal system and skin, regions where finer analysis of tissue structure is expected. Deeper structures are evaluated by using transducers with frequency in the range of 2 to $5 \mathrm{MHz}$.

The relation between frequency, resolution, and depth can be understood well, using the hypothetical example of two friends - an ant and an elephant (Fig. 8.23a, b). Both of them are commanded to perform the same tasks, the first being to pick up and bring back a crystal of sugar dropped at a distance of $100 \mathrm{~cm}$ from the start line and the second to pick up and bring back a log of wood placed 100 meters from the start line. Both of them proceed to perform the first task. The ant being small is able to easily identify the sugar crystal and get it back; however, the elephant keeps moving around unable to identify the crystal. As to the second task, the ant is able to travel only a short distance after which it dies of exhaustion, unable to reach the log of wood while the elephant travels the distance with ease and brings back the log of wood.

The ant is comparable to the high-frequency shortwavelength sound wave that is able to travel a short distance and bring back finer information about superficial tissues. If, on the other hand, it travels a long distance all its energy is absorbed by interaction with tissues. The elephant is comparable to the low-frequency sound wave with long wavelength that can travel deeper into the tissues and get information of deep structures, but limited tissue details. In a clinical setup, both the ant (high-frequency sound) and the elephant (lowfrequency sound) are essential for evaluation, depending on the clinical situation and region of interest.

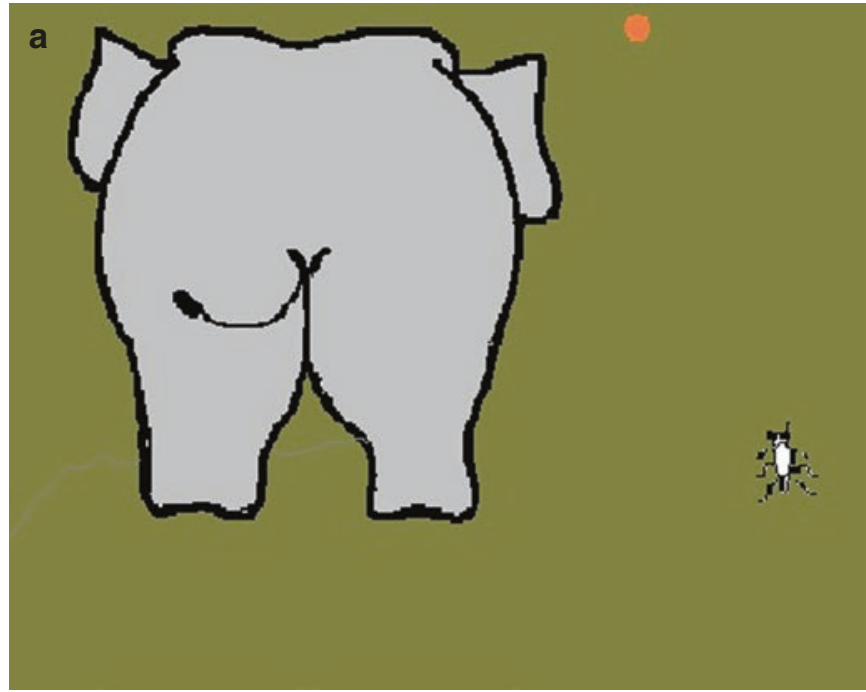

CAssociation of Oral and Maxillofacial Surgeons of India

Fig. 8.23 (a) and (b). Example of an elephant and an ant demonstrating their varying capability in picking up large and small objects from varying distances - a pictorial representation for understanding the dif-



ference in the penetration capacity of high- and low-frequency sound waves. (Explained in the text) 


\section{Color Doppler}

The display of flow of blood in a vessel is color coded. The flow toward the probe is depicted as red and flow away from the probe is blue. Arterial and venous flow, in a given anatomical region, is in opposite directions. Red generally represents flow in arteries, while blue represents venous flow. Turbulent flow is depicted using a mixture of colors.

\section{Power Doppler}

This display mode of Doppler conveys information about the overall flow in a given tissue, for instance, assessment of blood flow in a tumor. It helps to determine whether the tumor is hyper- or hypovascular.

\section{Strengths of Ultrasound}

- Ultrasound imaging is inexpensive and is free from the risk of radiation.

- Excellent soft-tissue resolution is possible and dynamic studies too can be performed under ultrasound guidance.

- It is the first line of investigation for hepatic, biliary, and renal disease and is an excellent modality for cyst imaging,

- Gynecological and prostate disorders are imaged using endocavitary ultrasound, Doppler studies allow assessment of blood flow, and real-time ultrasound imaging helps in guiding interventional biopsies and drainages.

\section{Weaknesses of Ultrasound}

- A long learning curve exists for some areas of expertise and image resolution is dependent on the machine that is available. Ultrasound does not give information of structure behind a bone and also gas filled structures.

\section{Specific Role of High-Resolution Ultrasonography (HRUSG) in the Maxillofacial Region}

HRUSG is a grossly underutilized modality in evaluating lesions of the maxillofacial region. This is due to the lack of awareness of its application. The tissues in the maxillofacial region are ideal for sonographic evaluation and hence is widely applied to help diagnose a number of clinical conditions. It can serve as the best possible initial parallel imaging investigation for all the superficial soft-tissue structures of head and neck region.

It is noninvasive and does not involve use of radiation. There are no contraindications to the use of ultrasound, and it can even be performed as a bed side examination. No prior specific preparation is required except for shaving of the beard, which might hamper the study. The study results are immediately available, and it is an excellent modality for evaluating various soft-tissue planes of the cheek. Evaluation of various anatomical spaces such as superficial masticator space, parotid space, carotid space, superficial neck spaces, visceral space, floor of the mouth, and all the infrahyoid neck spaces like the submandibular and sublingual spaces are possible using HRUSG, as are also pathologies like pre- and paravertebral abscesses, cellulitis, Ludwig's angina, and ranula [9].

High-resolution ultrasound is also capable of evaluating the nature of lymph nodes, assessing fluid and solid components and also assessing tissue vitality. Procedures like drainage of abscesses and biopsies can be conveniently performed under ultrasound guidance. Being a highly economical imaging modality, it is excellent for follow-up studies, excellent as a screening method, and at most times proves to be the conclusive examination [9].

\section{Drawbacks of Ultrasound Examination of the Maxillofacial Region}

Drawbacks include difficulty in assessing structures behind bony elements and structures containing gas. In case of huge masses, only partial information may be obtained, while shortnecked individuals and obese patients are difficult to evaluate. Structures at the base of the skull cannot be evaluated as are structures medial to ramus. Another limitation is that ultrasound cannot provide information about structures behind bone or any gas-filled structure. Scanning using a hockey stick probe might help overcoming this limitation. Scans need to be performed by individuals having vast experience and expertise.

There needs to be an increase in awareness regarding the developments in the application of HRUSG in the maxillofacial region and a close interaction between the surgeon and the sonologist for creating an atmosphere of better understanding among them.

\subsubsection{Thermography}

Medical thermography is used for early preclinical diagnosis and treatment of homeostatic imbalances. It is noninvasive and safe since it does not use any radiation. It makes use of body heat to diagnose a wide range of health conditions. By using high-speed computers and thermal imaging cameras, body heat is processed and recorded in the computer as an image map that can be analyzed. The analysis is based on determining the presence of abnormal hot and cold areas that can relate to different conditions.

\section{Applications in the Maxillofacial Region}

A variety of conditions related to blood flow in the head and neck vessels can be assessed by thermal imaging. Easy visualization of facial blood vessels by thermography is possible because of its superficial location. Common venues of thermographic use in the head and neck region include evaluation of pain related to differentiation of different types of headache, facial nerve injury following a blow to the face or an accident, and visualization of disorders of the temporomandibular joint. 


\subsection{Role of Chest Radiograph in the Practice of Maxillo Facial Surgery}

One of the most frequently requested investigations by an MFS is the Chest radiograph. Hence, interpretation of chest radiographic findings is of prime importance in determining the patient's clinical status.

\subsubsection{Chest Radiographic Evaluation for Optimum Diagnostic Quality}

An ideal chest radiograph, in terms of quality, is one that fulfills the following criteria (Fig. 8.24)

(a) Well-positioned patient without rotation.

- Purpose-Avoiding problems related to improper positioning that can obscure certain lung regions like the hila and mediastinal lines, can prevent clarity of borders, or can result in distorted position of structures like the trachea, which can be misinterpreted as a paratracheal mass.

- Ideal radiograph-The vertebral spinous processes are equidistant from the medial ends of the clavicle

(b) Proper lateral location of both scapulae on the radiograph.

- Purpose-avoids superimposition of scapulae over the lung fields

- Ideal radiograph-Medial border of the scapulae should be out of the lung field

(c) Inclusion of anatomical structures.

- Purpose-inclusion of entire lung fields from apex to diaphragmatic domes

- Ideal radiograph-must include the lower neck, both shoulders, lateral chest walls, and both diaphragms, including upper abdomen

(d) Proper identification references

- Purpose-Proper patient and radiographic verification.

- Ideal radiograph-must include the patient name, medical record number, date of acquisition, and the side marker (the most important of all)

(e) Optimum technical factors.

- Purpose-Clear visualization of the normal anatomy or presence of any pathology

\section{- Ideal radiograph-}

- Posterior third of ribs faintly visible through heart shadow

- Intervertebral spaces faintly visible through the heart shadow

- Obtained in deep inspiratory effort as revealed by position of diaphragms

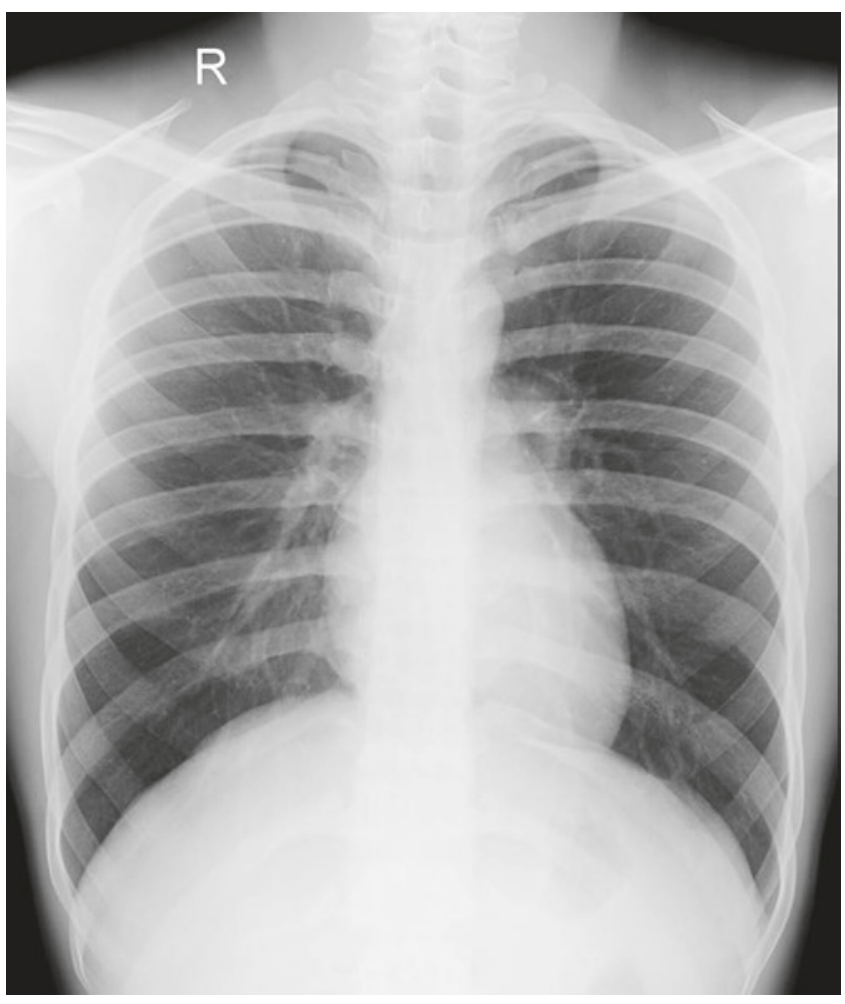

CAssociation of Oral and Maxillofacial Surgeons of India

Fig. 8.24 A Normal Chest Radiograph

\subsubsection{Reading a Chest Radiograph: Interpretation Basics}

The aim of reading a chest radiograph is to scan the whole image in a methodical way such that no anatomical part included in the radiograph is missed. While interpreting the radiograph, it is necessary for the interpreter to actually feel the structural component of the anatomical part being evaluated.

If, for example, a rib is being evaluated it needs to be traced, consciously keeping in mind, its component parts, the two cortical margins seen as white lines representing compact bone and the less dense central component representing marrow with fine normal trabeculae. This is in addition to evaluation of its number and shape. This meticulous evaluation will prevent missing of rib lesions and interpretation of the lesion is easier with prior knowledge of possible pathologies that affect ribs.

Many ways of methodical scanning have been documented in literature. The WHO-recommended method involves chest radiographic evaluation by beginning at one corner of the film, continuing to horizontally scan till the other corner, followed by sliding down and again continuing to scan toward the other edge, continuing till the whole of the radiograph has been scanned. As part of the examination, both sides of the chest must be compared.

It can be likened to the painting of a wall by a professional painter with a perfect finishing touch. Once the initial pri- 
mary scan is complete, specific areas called the lawyer's zone or hidden areas of the lung need to be looked over a second time. These areas include both the hilar regions, both cardiophrenic regions, both apical and clavicular regions, and finally also through the heart and diaphragms.

\subsubsection{Requesting a Chest Radiograph: Clinical indications}

\subsubsection{Preoperative evaluation}

i. Cleft lip and palate/any other congenital maxillofacial anomaly

ii. Infections of the maxillofacial region

iii. Known malignancy in maxillofacial region

iv. Trauma.

\subsubsection{Intra- / Postoperative recovery room}

i. Sudden choking and breathlessness

ii. Sudden cyanosis

iii. Sudden cough

iv. Chest pain.

v. Fever

vi. Evaluation of chest tubes and catheters (suggested reference for further reading).

\subsection{4 "The Chest Radiograph. A Mysterious Treasure"...the More you Search, the More you Find!!}

\subsubsection{Consolidation}

It is one of the most common terminologies used in Radiology. Clinicians and pathology textbooks, however, commonly use the term pneumonia. Any infection of the lung parenchyma is broadly referred to as pneumonia.
Involvement of the alveolar components of the lung parenchyma by the infection is called parenchymal consolidation [10]. It is a disease process in which the air normally present within the small lung air sacs (alveoli) is replaced by liquids like pus, blood, or other fluids, or solids like tumor cells.

Radiologically, consolidation appears opaque [11]. Normally, the alveoli are filled with air and so the normal opaque pulmonary vasculature can be visualized against the dark background of air. However, as discussed earlier, the replacement of the air spaces by any form of fluid or solid material makes the normally visualized pulmonary vessels inapparent with the air containing bronchi being visualized against the opaque background, which is called the air bronchogram (Fig. 8.25a-c).

The consolidation process involving a single lobe and limited by a fissure is called as lobar pneumonia, while the involvement of the entire lung is known as whole-lung consolidation. At times, the process is not as uniform and is distributed in the form of patchy opacities with intervening nonopacified alveoli, an appearance referred to as the air alveologram, which is seen in Bronchopneumonia.

\section{Pulmonary Edema}

Maxillofacial surgeons are often faced with the problem of patients going into a state of sudden breathlessness in the postoperative recovery room for which the MFS asks for a portable chest radiograph. One of the most common causes of sudden breathlessness in the postoperative recovery room is pulmonary edema. The common confusion for which MFS postgraduates seek the radiologist's opinion is whether the cause of breathlessness is bronchopneumonia or aspiration bronchopneumonia.

In case of acute cardiogenic pulmonary edema caused either due to left ventricular failure or fluid overload, the characteristic radiographic abnormality would reveal bilateral symmetrical areas of consolidation predominantly in the


CAssociation of Oral and Maxillofacial Surgeons of India

Fig. 8.25 (a) and (b) Consolidation: Homogeneous opacities with "Air-bronchogram" sign. (c) Schematic diagram showing group of alveoli filled with fluid replacing normal air 



CAssociation of Oral and Maxillofacial Surgeons of India

Fig. 8.26 (a) Congestive cardiac failure with cardiomegaly and pulmonary edema. (b) Resolution of pulmonary edema after treatment

perihilar and lower lung zones [10] (Fig. 8.26a, b). Noncardiogenic pulmonary edema caused by different pathologies, including fluid overload, acute glomerulonephritis and adult respiratory distress syndrome among others, affects the permeability of the pulmonary capillary membrane (Fig. 8.27). The distribution can vary depending on the position of the patient. The opacities seen on the portable radiograph should not be confused with the appearance of pneumonia (Fig. 8.28).

Rare causes of unilateral pulmonary edema, including raised intracranial pressure and the administration of fluid through the central venous catheter inadvertently placed in the pulmonary artery, need to be kept in mind as well.

\section{Atelectasis}

Diminished air within the lung associated with reduced lung volume is termed atelectasis manifesting radiologically as pulmonary opacity accompanied by volume loss. The causes are varied, including resorption, relaxation, adhesive and cicatrization atelectasis. Of these, the form of atelectasis a maxillofacial surgeon commonly deals with is resorption atelectasis, where there is an obstruction in the communication between the alveoli and trachea. The obstruction may be at any level from the common air way to the segmental bronchus; however, common air way obstruction resulting in bilateral atelectasis is a rare occurrence [11]. Radiologically, the appearance is that of a homogeneous opacity without an air bronchogram sign, with loss of lung volume and displacement of structures toward the opacity.

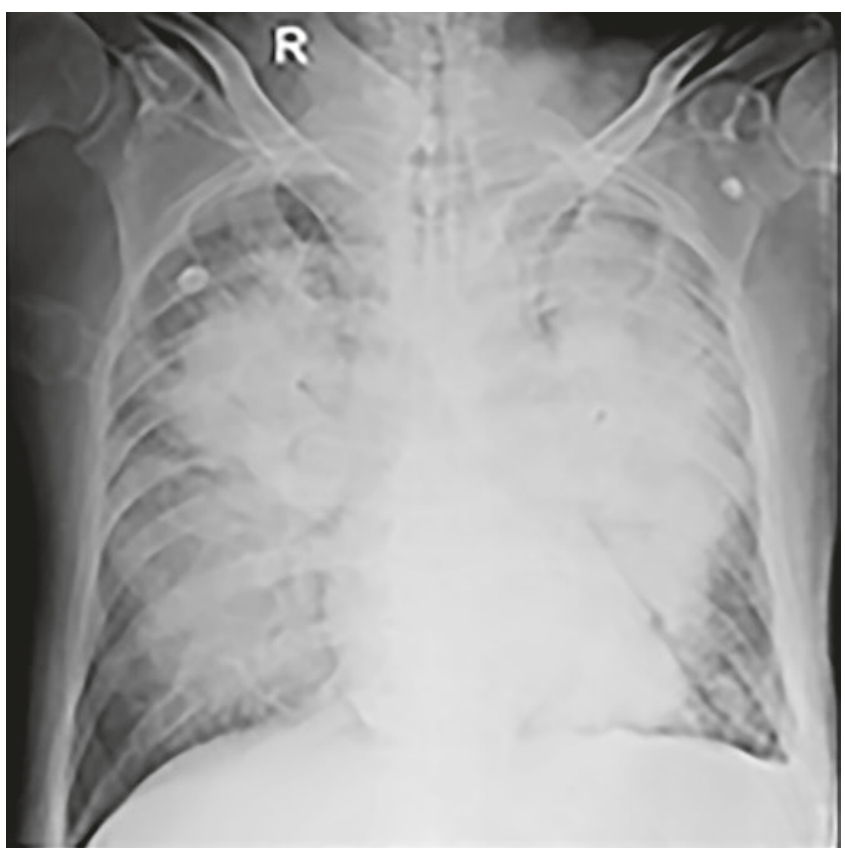

CAssociation of Oral and Maxillofacial Surgeons of India

Fig. 8.27 Noncardiac pulmonary edema in a patient with renal failure

The maxillofacial surgeon commonly comes across similar radiographs in the postoperative recovery room. The commonest cause of this is the inadvertent placement of the endotracheal tube into one bronchus, causing occlusion of the other bronchus, and resulting in atelectasis as a 


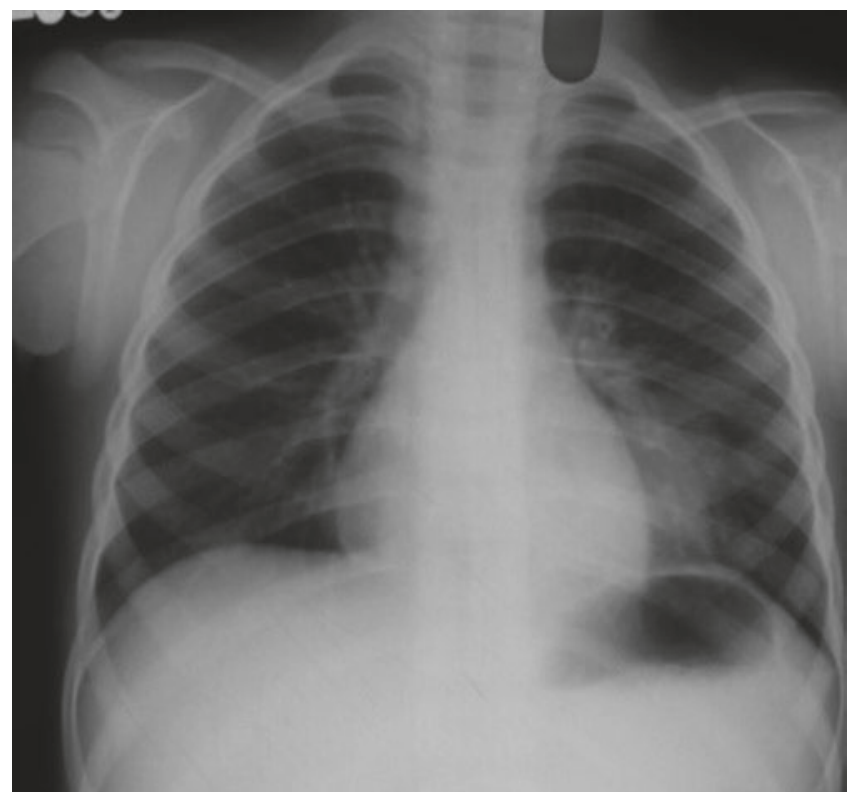

CAssociation of Oral and Maxillofacial Surgeons of India

Fig. 8.28 Aspiration Bronchopneumonia

consequence. Early detection and diagnosis with readjustment of the endotracheal tube is bound to be life saving, helping prevent long-term complications (Fig. 8.29a-c). Thick mucus plugs are other common occluding elements, seen in postoperative recovery rooms resulting in lobar or segmental opacities with loss of volume. Again, early detection and good respiratory physiotherapy are keys to improved lung compliance with a radiograph taken at a later date as follow-up.

\section{Relaxation Atelectasis}

Normally negative intrapleural pressure is caused by competing thoracic pressures. Lung elasticity and surface tension of the alveolar fluid create an inward tension, pulling the lung inward, which is countered by opposing forces from the pleural fluid and thoracic wall, the pleural cavity surface tension, and the parietal pleural attachment to the thoracic wall causing outward pull of the lungs. The outward pull is slightly higher than the inward pull with a negative intrapleural pressure of about $-4 \mathrm{~mm}$ of $\mathrm{Hg}$. When this intrapleural negative pressure is lost as occurs commonly in cases of pneumothorax or pleural effusion, the lung has a natural tendency to recoil, with the loss of lung volume being proportional to the amount of air or fluid collected in the pleural space [11].

\section{Pneumothorax}

The presence of air in the pleural space is called pneumothorax, the most common cause of which is trauma either accidental or iatrogenic. In the absence of such causes, it is called spontaneous pneumothorax. Radiological findings of pneumothorax include increased peripheral translucency, absence of lung markings within this area of translucency, and the identifiable visceral pleural margin of the collapsed lung (Figs. 8.30 and 8.31). The detection of a pneumothorax should prompt the search for an underlying cause like a fractured rib or a ruptured bulla or iatrogenic causes like a central venous catheter procedure. Associated lesions like pneumomediastinum and surgical emphysema should also be looked for.

A maxillofacial surgeon might have to read supine portable radiographs of patients presenting with clinical signs suggesting respiratory distress and the presence of pneumothorax might be missed. In such a scenario, the MFS should look for radiographic evidence of pneumothorax at sites adjacent to the diaphragmatic silhouettes on either side and the cardiophrenic and costophrenic angles and he should also be able to appreciate the appearance of an exceptionally sharp cardiac border [11]. The MFS needs to be aware that air trapped within the skin folds can mimic pneumothorax (Fig. 8.32).

Identification of an air fluid level would prompt the diagnosis of a hydropneumothorax. It is difficult to characterize the nature of the fluid as being transudate, exudate, blood, or chylous. An MFS may be unable to diagnose a hydropneumothorax, on an AP supine portable radiograph as the fluid level will not be identified. Here, the collapsed lung tends to float on the fluid, which is dependent posteriorly giving a veil effect (Fig. 8.33).

\section{Pneumomediastinum}

It is the collection of air within the pleural boundaries of the mediastinum and can arise secondary to barotrauma from mechanical trauma, intrathoracic, or iatrogenic trauma and infection or can occur spontaneously. A radiographic diagnosis of pneumomediastinum requires depiction of normal anatomic structures being outlined by air as they leave the mediastinum. The mediastinal air can cause elevation of the thymus, collect anterior or posterior to the pericardium, surround the pulmonary artery or its branches, or can collect adjacent to the major aortic branches or major bronchi (Fig. 8.34).

Most cases of pneumomediastinum described in maxillofacial surgery literature are as a result of dissection of air down the fascial planes of the neck. Chest radiographic findings need to be carefully examined to make the diagnosis of pneumomediastinum, which is crucial to the MFS in planning appropriate treatment of affected patients. On rare occasions, a significant amount of air in the mediastinum can cause vessel or tracheal obstruction and induce symptoms and signs of tamponade and decreased venous return. 



CAssociation of Oral and Maxillofacial Surgeons of India

Fig. 8.29 (a) Whole lung collapse: Left hemithorax is homogenously opaque with loss of volume of left lung with displacement of mediastinum to left. Note: Abnormal placement of endotracheal tube blocking the (Lt.) Bronchus. (b) Schematic diagram showing loss of volume of alveoli as seen in collapse with absence of air. (c) Reversion of normal left lung aeration after repositioning of endotracheal tube 


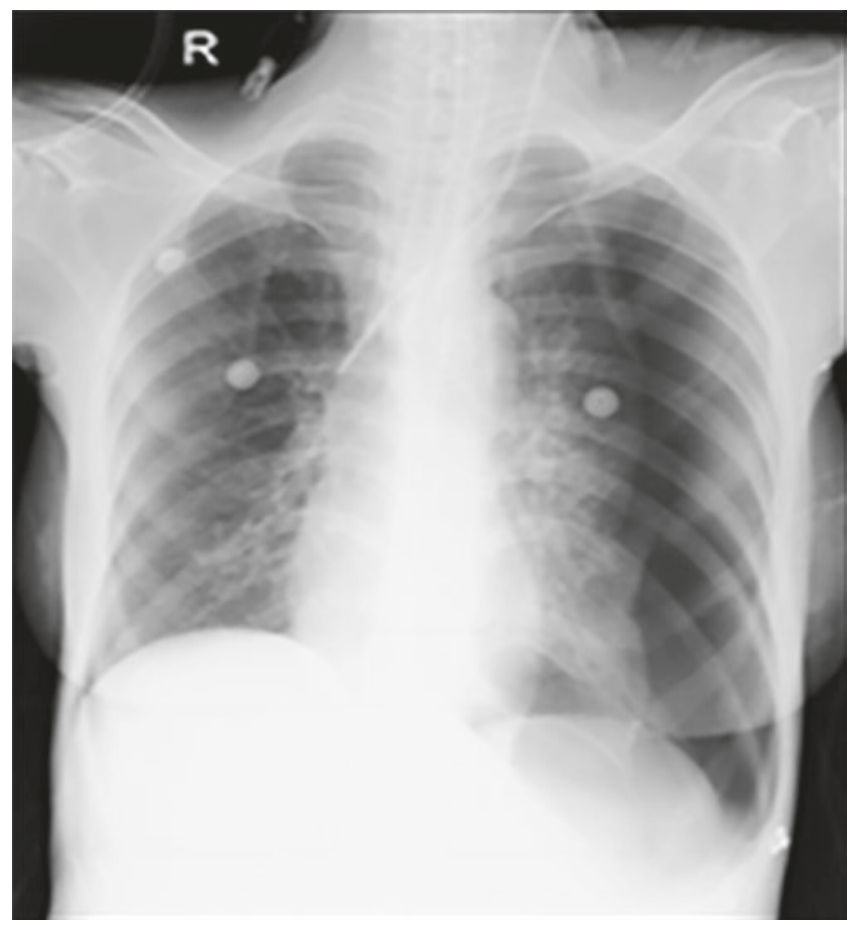

CAssociation of Oral and Maxillofacial Surgeons of India

Fig. 8.30 Left-sided pneumothorax

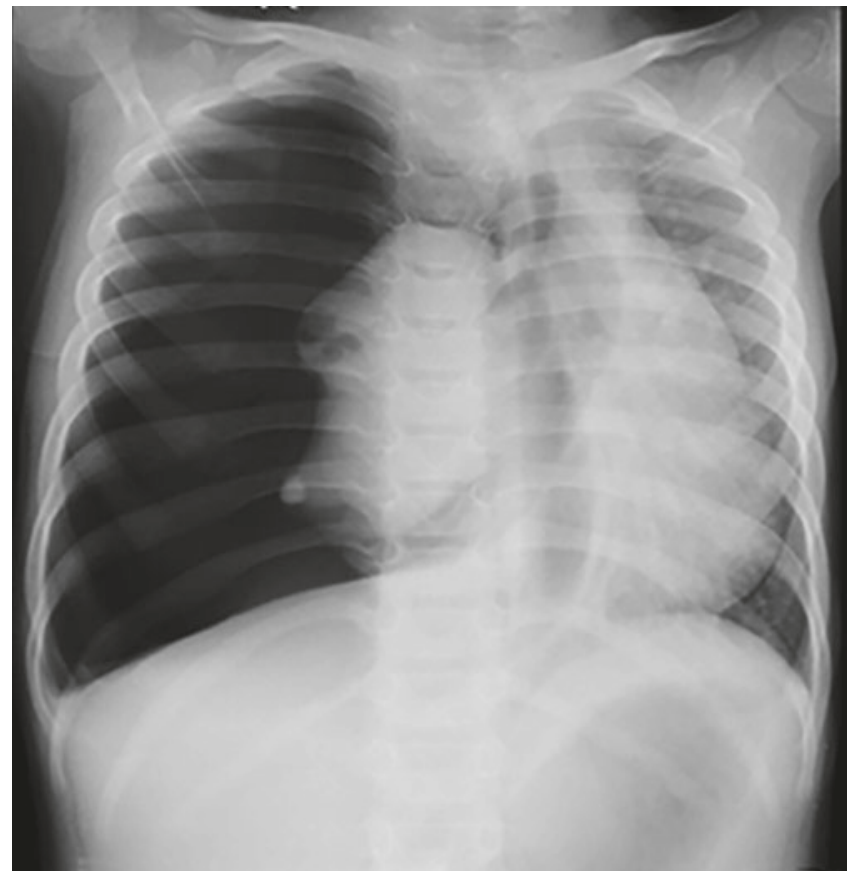

CAssociation of Oral and Maxillofacial Surgeons of India

Fig. 8.31 Right-sided tension pneumothorax

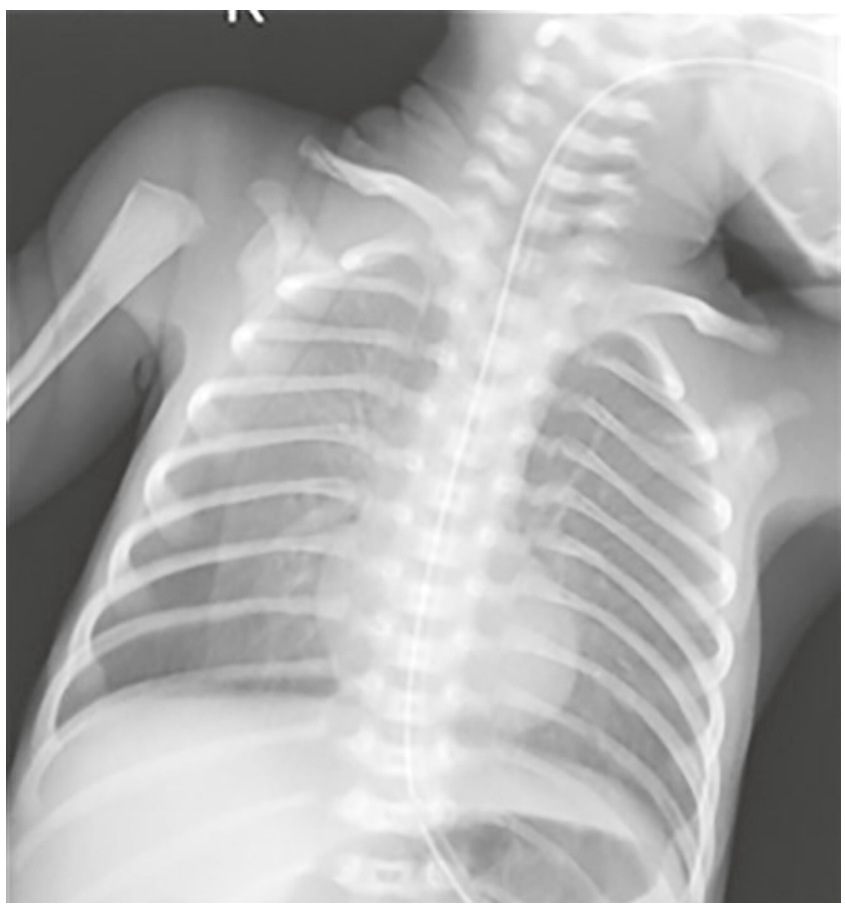

CAssociation of Oral and Maxillofacial Surgeons of India

Fig. 8.32 Right-sided pseudopneumothorax due to air trapped in skin fold

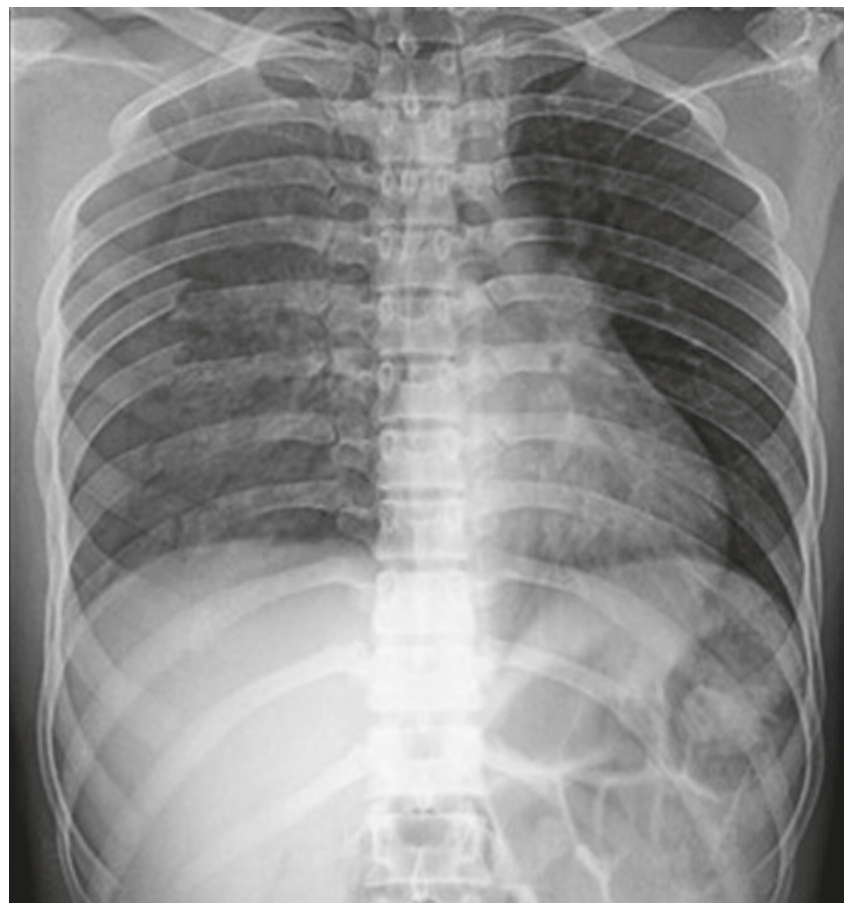

CAssociation of Oral and Maxillofacial Surgeons of India

Fig. 8.33 Right-sided hemopneumothorax with multiple rib fractures as seen in supine radiograph 


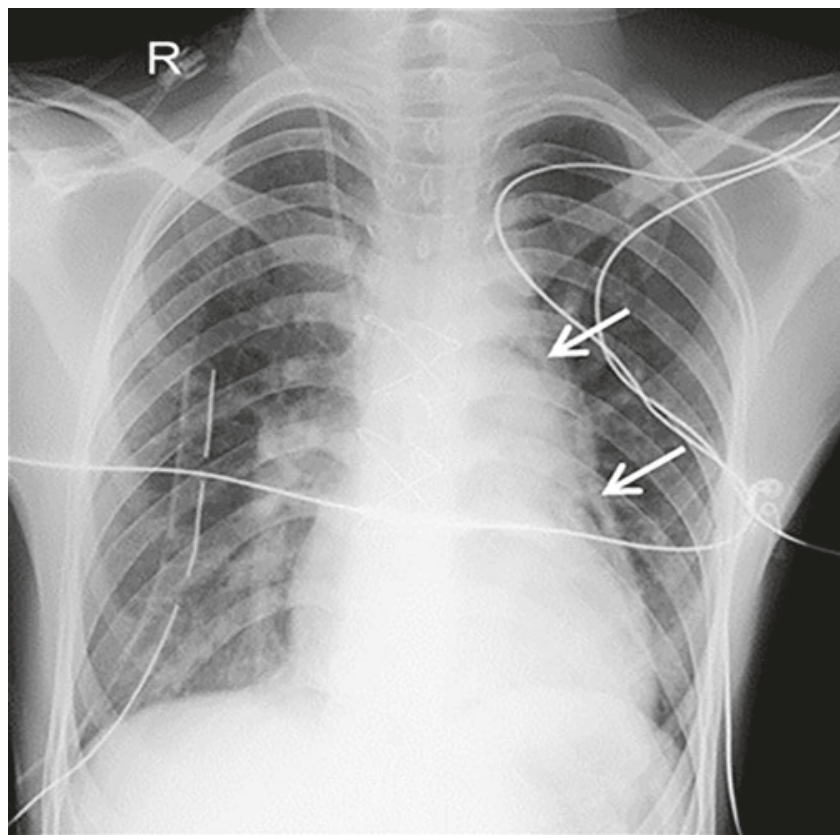

CAssociation of Oral and Maxillofacial Surgeons of India

Fig. 8.34 Pneumomediastinum. Air around the pulmonary artery and heart.

Fig. 8.35 (a) and (b). Surgical emphysema with

\section{pneumothorax}

\section{Surgical Emphysema}

Though subcutaneous emphysema or surgical emphysema actually means gas in the subcutaneous tissues, it also includes soft-tissue emphysema that dissects into the deeper soft tissues and musculature along fascial planes. The translucent air spreads over the chest wall and axilla into the root of the neck. Fascial planes clearly outline the pectoralis muscle and neurovascular bundles in neck (Fig. 8.35a, b). If trauma is the cause, the gas by itself may not need treatment, but its identification is of importance as it may be the only indication of the presence of other serious injuries requiring urgent management. Surgical emphysema can uncommonly occur as a serious complication of oral and maxillofacial surgical procedures. It may also occur following teeth extraction, endodontic treatment, or procedures like restorative dentistry, periodontal and temporomandibular joint surgery, or facial fracture repair.

\section{Pulmonary Nodules}

For all intents and purposes, a nodule seen in the lung (especially in the lower zones) should be considered as metastasis unless proven otherwise, by a maxillofacial surgeon.


(CAssociation of Oral and Maxillofacial Surgeons of India 
Pulmonary nodules can be characterized, based on some signature findings. In the absence of these findings, the nodule remains nonspecific. Common causes of pulmonary nodules are granulomas, bronchogenic cysts, hydatid cysts, hamartomas, pulmonary hematoma following laceration, rheumatoid nodules, fungal granulomas, and pulmonary metastasis.

Tuberculomas usually range in size from 1 to $3 \mathrm{~cm}$ in diameter, have central calcific lesions, and are surrounded by satellite scarred lesions. They are commonly seen in the upper zones, but they can occur anywhere in the lung (Fig. 8.36).

Bronchogenic cysts are well-defined nodules, around 3 to $4 \mathrm{~cm}$ in size, usually seen in the parahilar region, and are sometimes purely in the mediastinum (Fig. 8.37a-e).

Hamartomas are nodules that can occur anywhere in the lung having well-defined margins with central popcorn-like calcification (Fig. 8.38a, b).

Hydatid cysts, too, can occur anywhere in the lung. They have a stenciled outline and vary in size from 1 to $10 \mathrm{cms}$. The radiographic air crescentic sign is pathognomonic of a hydatid cyst, appearing like the arc of the moon caused by the separation of the endocyst from the exocyst. Continued separation of the endocyst from the exocyst results in an air fluid level typically called the 'water lily' appearance. Pulmonary hydatid cysts do not calcify.

Presence of a solitary nodule in the lower lung without any characteristic findings makes pulmonary metastasis a strong possibility, which should be further evaluated with imaging and HPR. Multiple nodules of varying sizes in the lower zones are very much suggestive of pulmonary metastasis.(Fig. 8.39a, b).

\section{Role of Chest Radiograph in Cases \\ Developing Sudden Onset of Breathlessness in Postoperative Period}

1. Aspiration Bronchopneumonia

2. Pneumothorax

3. Foreign body (Fig. 8.40a-c)

4. Abnormal placement of Endotracheal Tube (Fig. 8.41)

5. Pleural effusion

6. Pulmonary edema and pulmonary embolism

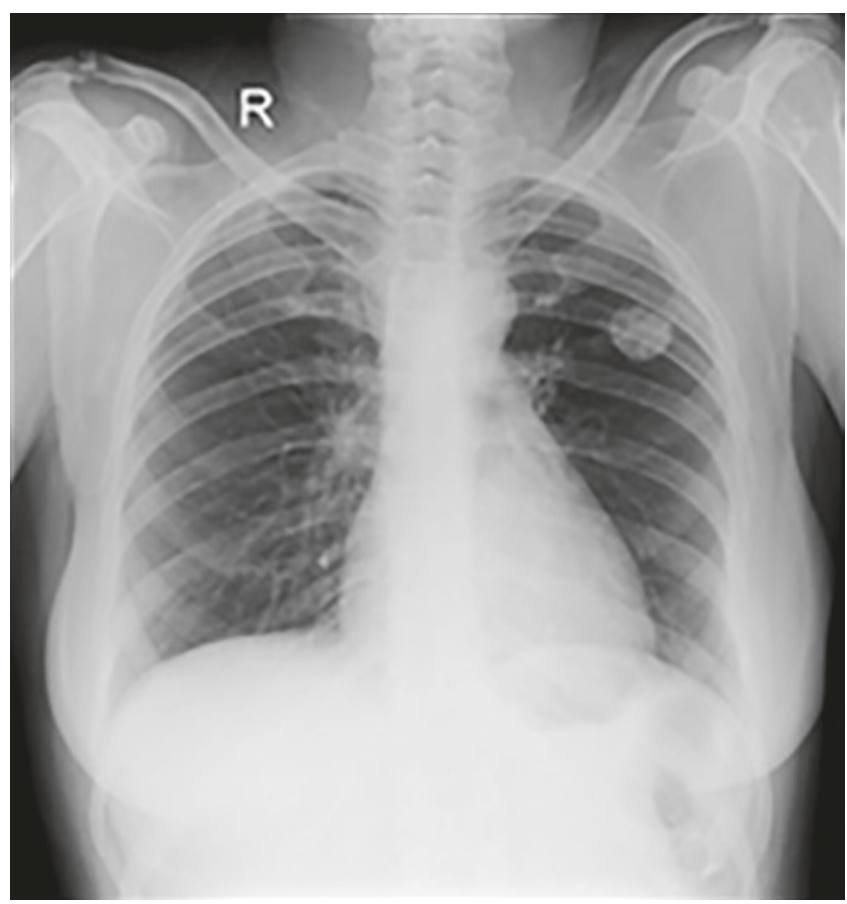

CAssociation of Oral and Maxillofacial Surgeons of India

Fig. 8.36 Pulmonary Nodule-Tuberculoma

A maxillofacial surgeon should be capable of identifying all of these causes of sudden onset of breathlessness in a postoperative patient in the event of the absence of availability of expert radiological opinion and act accordingly. They also need to be aware that patients on ventilators may develop ventilator-dependent complications.

\section{A glance into the future of imaging for Maxillofacial Surgeons}

1. Magnetization Transfer Imaging

This is a modified MRI study that helps in

- Prediction of whether the primary tumor is benign or malignant (possible in 80 to $90 \%$ cases).

- Differentiation of reactive versus malignant adenopathy.

This modality is still in the preliminary stage and needs further study.

2. MRI-guided biopsy procedures need MRI compatible equipment. The procedures are feasible and are usually done in cases that are not amenable to USG guidance.

3. Functional imaging of swallowing. 




Fig. 8.37 (a), (b), and (c). Preop chest radiographs of a patient with cleft lip presenting with dyspnea, more with changing position. (d) and (e) Mediastinal mass-Bronchogenic Cyst confirmed on CT 
Fig. 8.38 (a) Pulmonary

Nodule-Hamartoma. (b)

Confirmed on CT

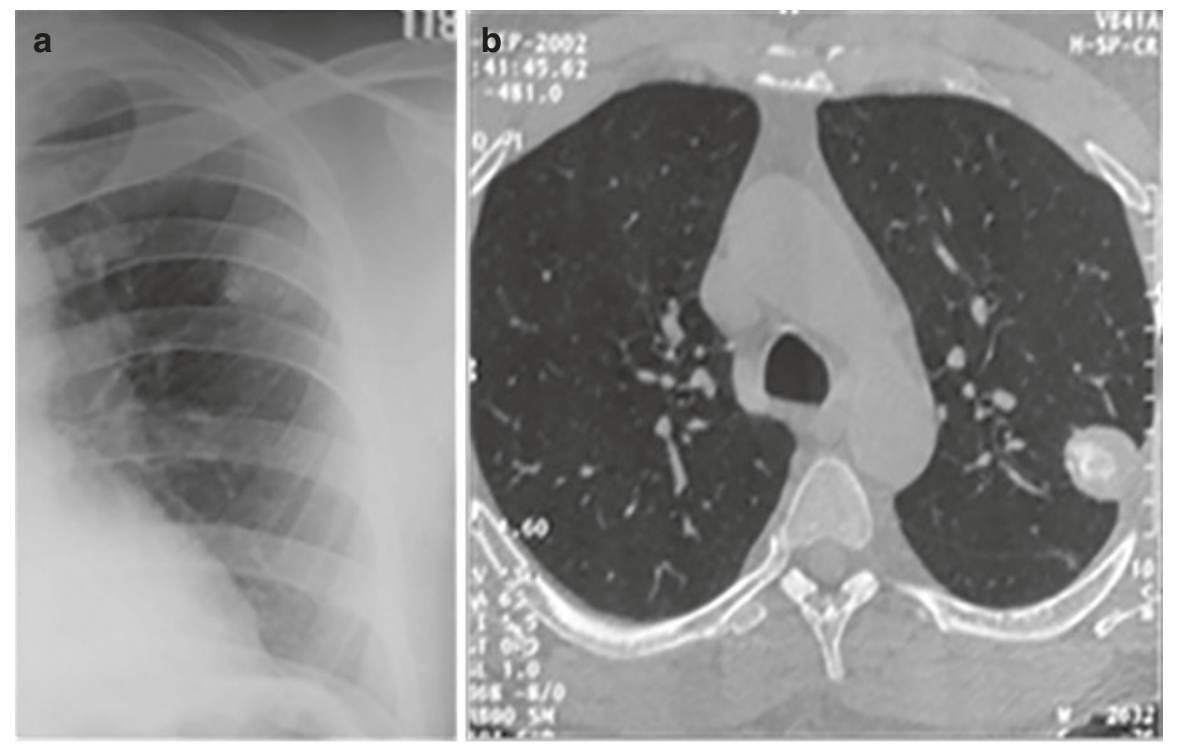

(C)Association of Oral and Maxillofacial Surgeons of India
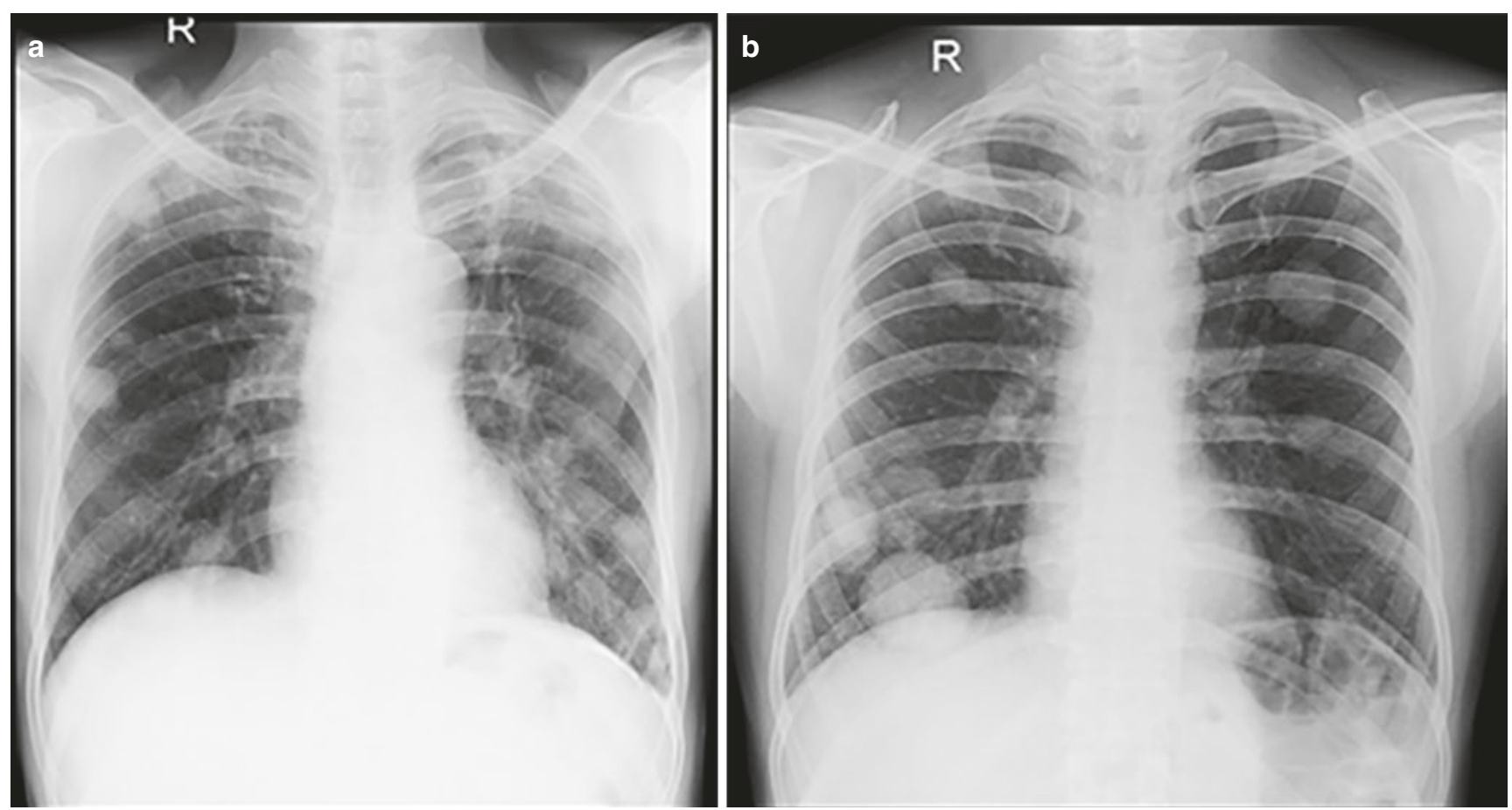

CAssociation of Oral and Maxillofacial Surgeons of India

Fig. 8.39 (a) and (b). Pulmonary metastasis 

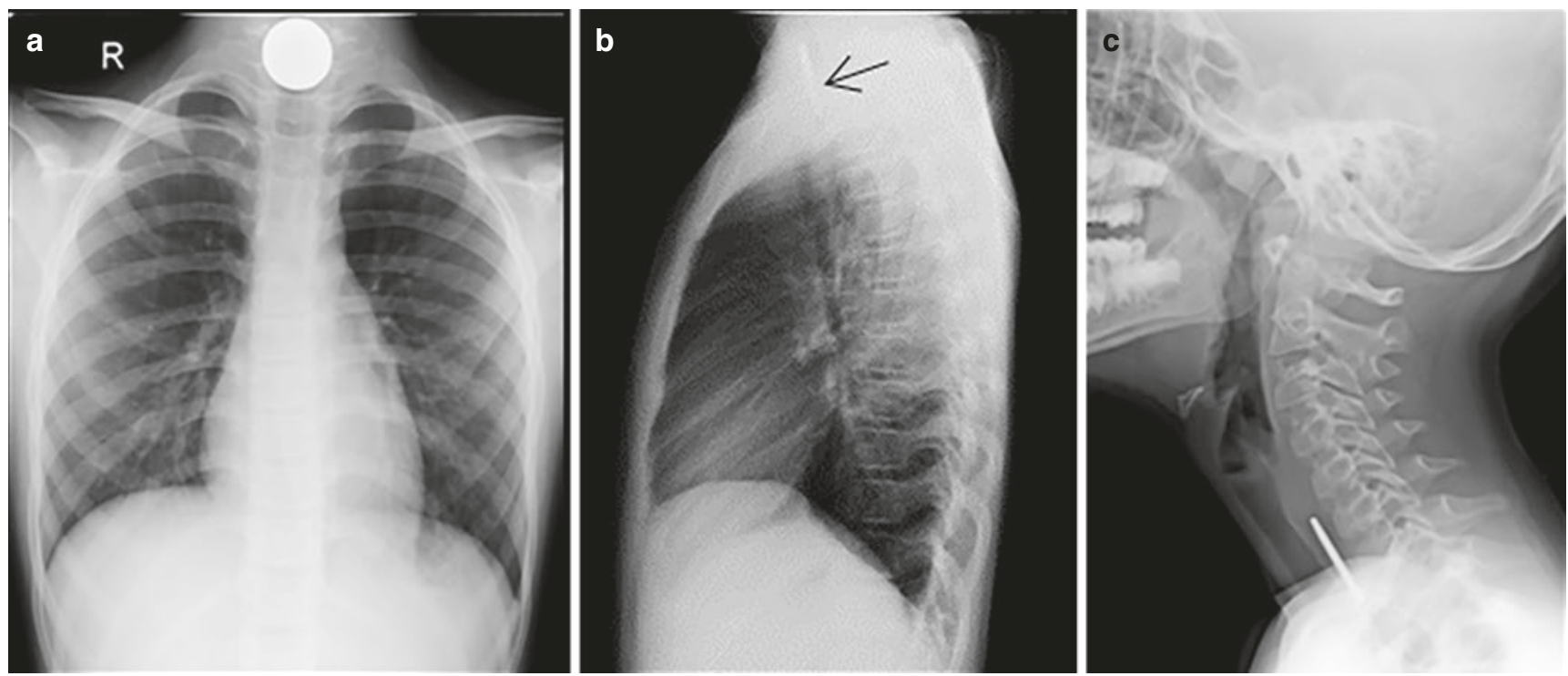

CAssociation of Oral and Maxillofacial Surgeons of India

Fig. 8.40 (a), (b), and (c). Foreign body, a coin in the cervical esophagus



CAssociation of Oral and Maxillofacial Surgeons of India

Fig. 8.41 Abnormal placement of endotracheal tube with collapse of left lobe

\subsection{Role of High Resolution Ultrasonography (HRUSG) as a Diagnostic Aid in the Practice of Maxillofacial Surgery}

\subsubsection{Preface}

Primarily the use of HRUSG in the maxillofacial region is directed toward investigation of the cheek and the structures adjacent to it. Despite the layers of the cheek and the adjacent structures having a classical sonographic appearance, literature available on HRUSG of the cheek is limited. Having proper understanding of the relevant anatomy is critical in discerning the wide range of diseases affecting this region. Evaluation of these lesions by ultrasound is possible only once the normal ultrasound anatomy at various levels is defined and a comparison of the suspicious lesion with the normal expected appearance is made. An original attempt toward this goal was made at our institute with comparison of normal cadaveric sections at corresponding levels taken by HRUSG.

High-resolution ultrasonography has broad applications in the maxillofacial region especially in the cheek, which 
include evaluations of the unilateral or bilateral swollen cheek painful or pain less, restricted mouth opening, painful chewing without obvious clinical findings, and varied causes of facial swelling. Since early detection decisively influences patient prognosis in case of malignancy, it is one of the prime concerns in a presenting patient. Further, differentiation of lesions as being of benign, malignant, infective, or inflammatory etiology is important in deciding on a treatment plan. HRUSG should however be used to complement and not replace other imaging modalities as it does have its limitations.

Compared to other facial components, the cheek is predominantly composed of adipose tissue and is relatively small in size. Swelling of the cheek is easily visible and palpable in most patients presenting with cheek masses. Most lesions have nonspecific characteristics on computed tomography $(\mathrm{CT})$ and magnetic resonance imaging (MRI). In contrast, HRUSG is ideal for evaluation of cheek lesions, because of its capability to differentiate between tissues like skin, fat, muscle, gland, ducts, and vessels especially since bony and gaseous interfaces are absent. Further, the superficial location of the cheek makes it easily accessible to ultrasound. Vestibule can be evaluated with fruit jelly technique obliterating the void space.

This short review attempts to aid the MFS in his understanding of HRUSG of the maxillofacial region, with special reference to the cheek.

\subsubsection{Gross Cheek Anatomy}

In simple terms, the cheek is the fleshy portion of the face, below the eye, extending from the angle of the mouth to the ear. Anatomically, it pertains to the buccomasseteric region comprising the buccal space and its contents, masseteric and buccinator muscles, the buccal fat pad, and Stenson's duct.

The region is continuous anteriorly with the lips. Its external anterior demarcation is formed by the nasolabial fold and groove extending from the side of the nose to the angle of the mouth. The mucosa lining the inner aspect of the cheek adheres to the buccinator muscle, while the gingival mucosa covers the mandibular and maxillary alveolar processes. The gingivobuccal sulcus is the junction formed between the gingival and buccal mucosae. The vestibule is the region of the mouth between the teeth, lips, and cheek, which is bound superiorly and inferiorly by buccal mucosal reflections onto the mandible and maxilla. Posteriorly the vestibule is continuous with the oral cavity proper (Fig. 8.42) [12, 13].

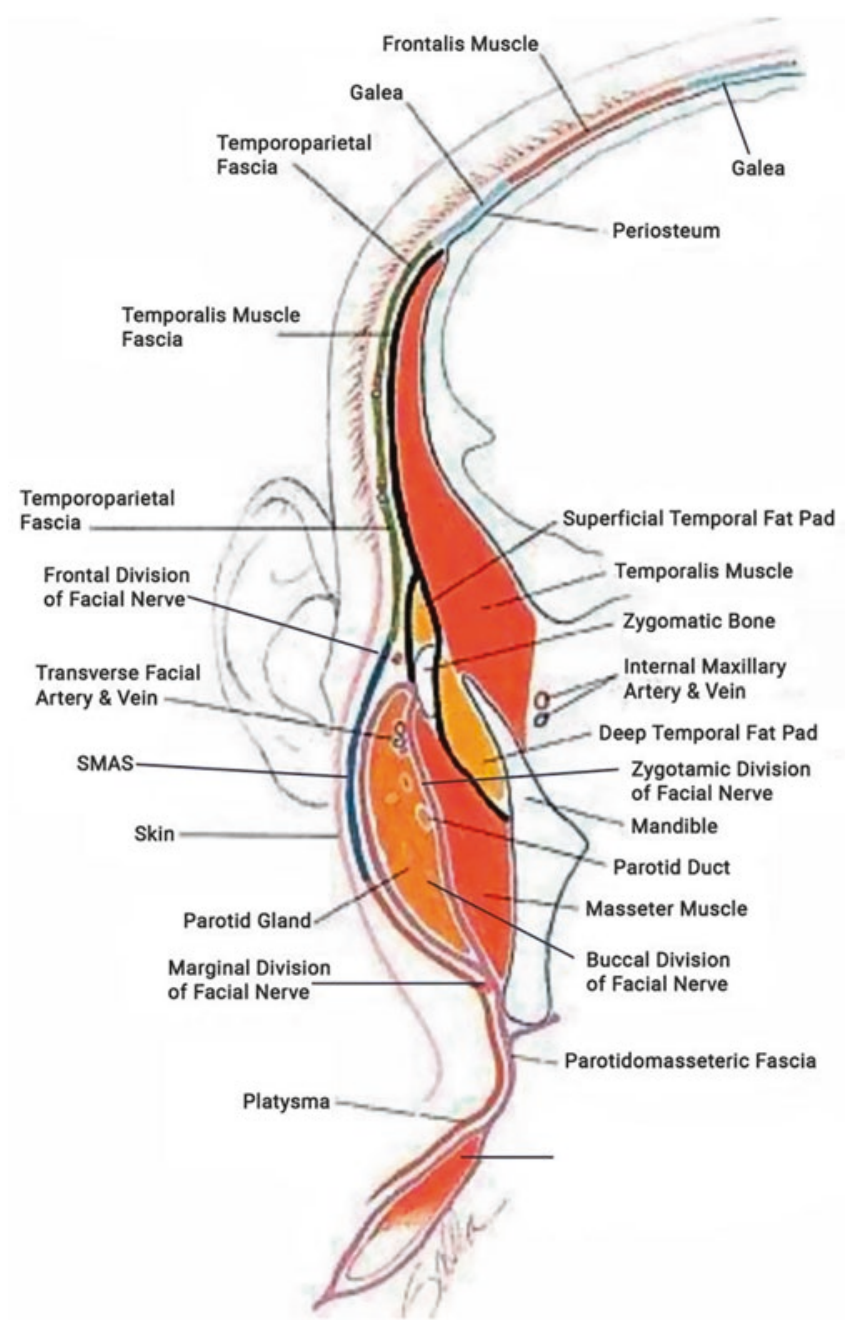

CAssociation of Oral and Maxillofacial Surgeons of India

Fig. 8.42 Anatomical layers of the cheek

\subsubsection{Floor of Mouth, Anatomy (Relevant to HRUSG)}

The part of the oral cavity located underneath the tongue is called the floor of the mouth and it can be involved by a myriad of pathological processes. Superficial lesions involving the mucosal surface are easily visualized and may not need imaging. Disease involving the deeper structures of the floor needs to be evaluated by imaging, and HRUSG is many times the first line of investigation because of its superior soft-tissue differentiation capability, thus allowing for clear depiction of contents of the floor [14]. 
Inflammatory processes, malignancies, and vascular abnormalities can affect the floor contents, while certain conditions like ranula and obstruction of the submandibular gland are specific to this location [14].

\subsubsection{Defining the HRUSG Anatomy of Maxillofacial Region (Predominantly Cheek)}

\section{Normal Ultrasound Cheek}

Various cheek layers have varying sonographic echogenicity and echotexture, and hence ultrasound is an invaluable tool in the characterization and localization of cheek lesions.

Depending on tissue density, the sound wave transmission, absorption, or reflection capability of each tissue varies. Tissues, having high water content like blood, appear black and are called anechoic because they conduct sound waves well. In contrast, tissues that are poor conductors reflect most of the sound wave energy back to the transducer appearing bright and are termed hyperechoic, examples of which include bones, tendons, and fascial planes. Tissues like muscle reflect less of the sound wave energy and have a hypoechoic appearance. In the cheek, the mucosal and submucosal layers appear hyperechoic, the mucosa more so. Muscle tissue appears hypoechoic, while fatty tissue appears echogenic (Fig. 8.43).

Compared to other imaging modalities, ultrasound has proven to be superior as regards identification of small lesions, detection of lesion plane, and lesion characterization (Fig. 8.44).

The cheek layers and neighboring structures that can be identified by HRUSG are.

\section{Cheek}

- Skin

- Subcutaneous plane

- Buccal pad of fat

- Buccinator muscle

- Submucosal layer

- Mucosal layer

- Masseter muscle and its lateral relations.
- Temporalis muscle

- Temporomandibular joint (TMJ)

Neighboring Structures

- Vestibule

- Gingiva

- Buccogingival Sulcus

- Periodontal tissue, etc.

- Tongue, lips

- Floor of mouth, Sublingual space

- Submandibular space

- Parotid gland / duct

- Retromandibular space

- Interdental space

- Alveolar margin

- Mental foramina

- Infraorbital foramina

- External nose

- Various other facial planes and spaces

- Arteries

\subsubsection{Techniques of HRUSG of Cheek}

For ultrasound examination, the cheek is considered to be a rectangular area bordered as follows (Fig. 8.45).

- by an imaginary line beginning at the angle of mandible and extending along its lower border to the level of angle of mouth inferiorly,

- by an imaginary line beginning at the angle of mandible and running along the posterior border of the mandibular ramus to include the temporomandibular joint posteriorly,

- by an imaginary line along the zygomatic arch superiorly and,

- by an imaginary vertical line at the level of angle of mouth that joins the superior and inferior lines anteriorly [15].

Various techniques, including the resting (neutral) and Puffed (Blow out) cheek technique, the tongue touch technique, and fruit jelly technique, were experimented with, each having their own advantages and limitations [15].
Fig. 8.43 Normal Cheek/ Buccal layers on ultrasound

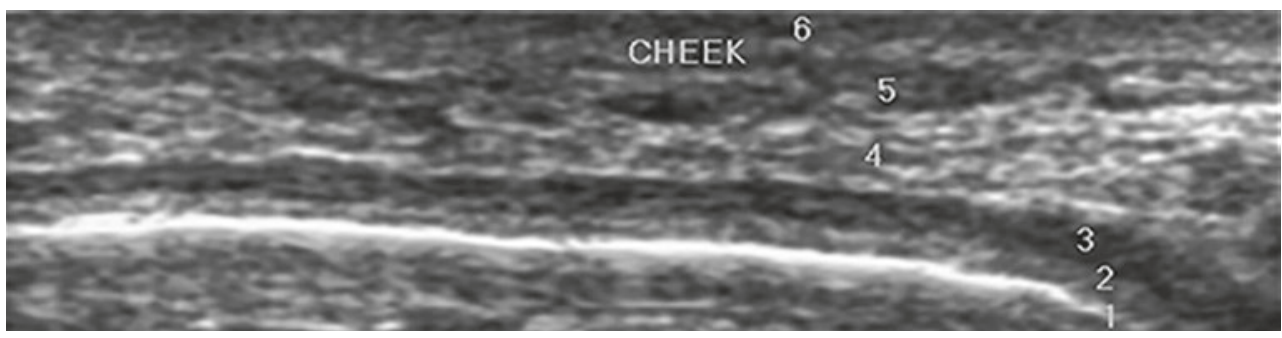

CAssociation of Oral and Maxillofacial Surgeons of India 

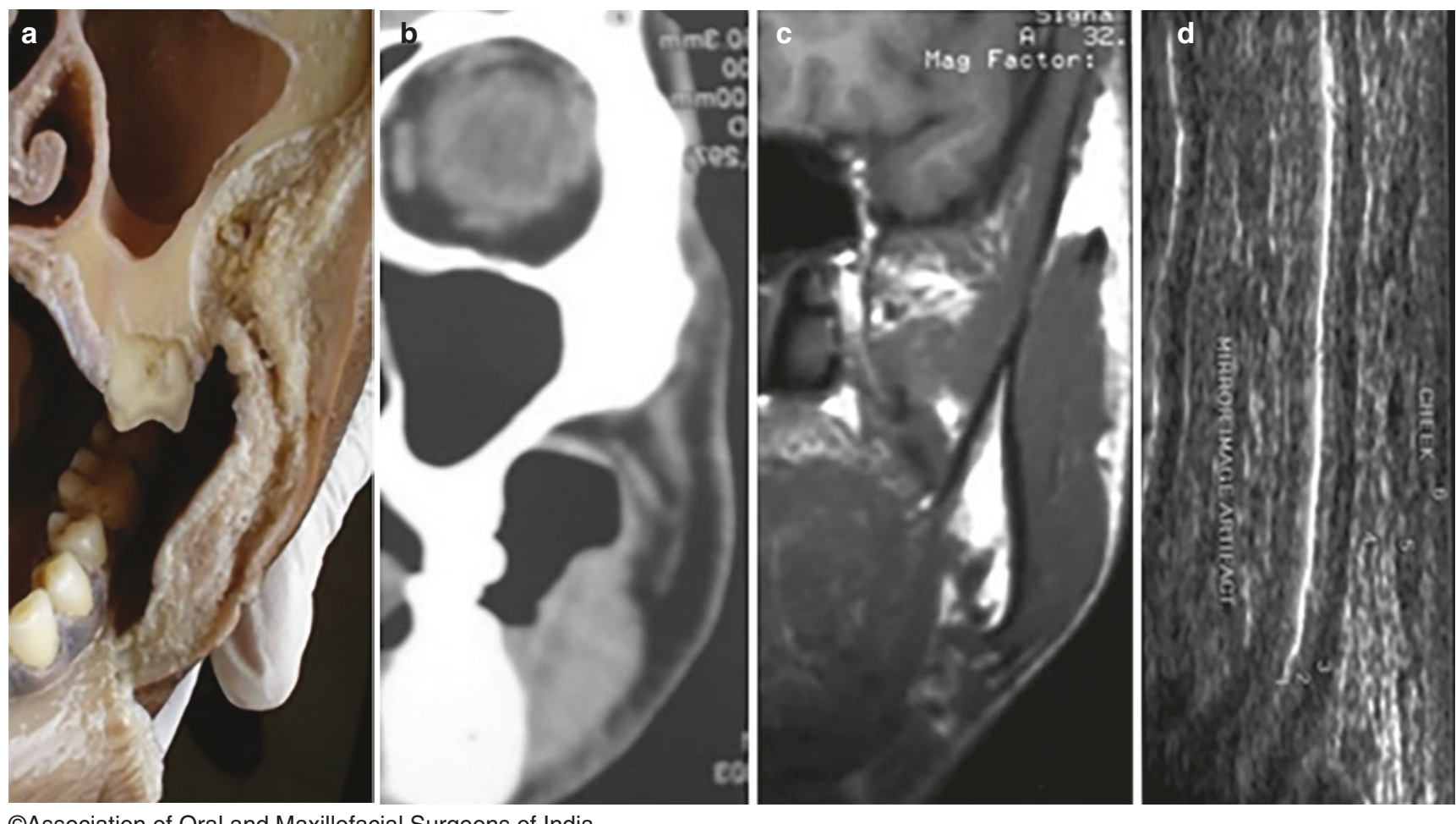

CAssociation of Oral and Maxillofacial Surgeons of India

Fig. 8.44 (a-d) Cheek anatomy - a comparison. Comparative cheek anatomy- (a) cadaveric specimen, (b) CT, (c) MRI, and (d) Ultrasound

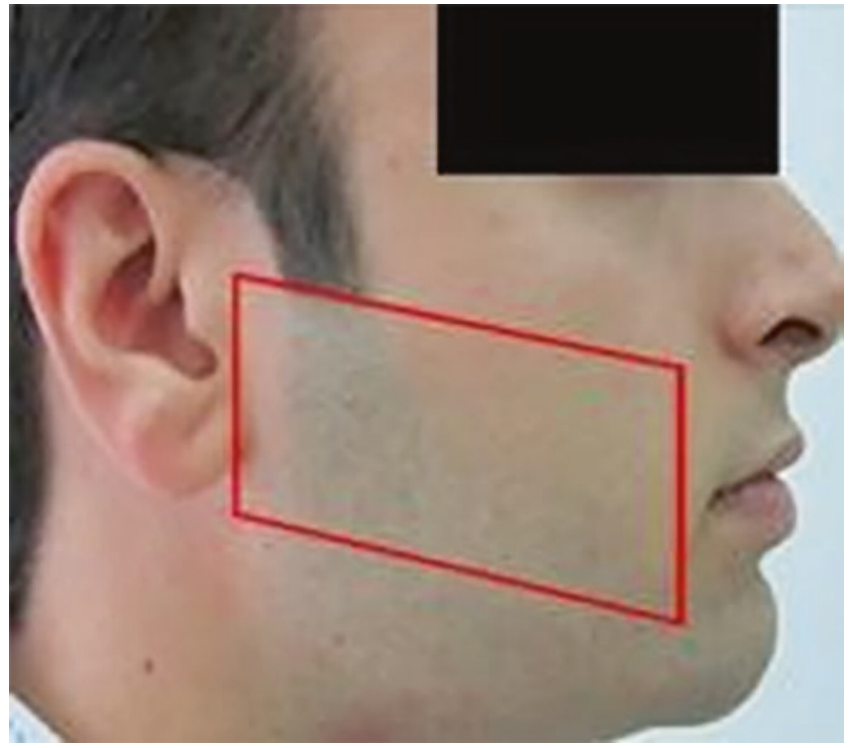

CAssociation of Oral and Maxillofacial Surgeons of India

Fig. 8.45 Area of Cheek ultrasound study

(a) Resting (Neutral) and Puffed (Blow out) Cheek Technique (Figs. 8.46 and 8.47).

- Patient is instructed to blow, and ultrasound is performed in puffed cheek status.

- Images obtained in axial and coronal planes. Advantage: Improved visibility of cheek layers and accurate definition of lesion size.
Limitation: Occurrence of mirror image artifact. Less sensitivity in evaluation of lesions of the vestibule (gingivobuccal sulcus).

(b) Tongue Touch Technique (Fig. 8.48a-c).

- Patient is instructed to feel the cheek lesion with the tongue.

- Lesion is brought closer to the probe creating a better acoustic window.

- Lesions of the superficial buccal mucosa and tongue lesions on its anterior half and corresponding lateral borders can be evaluated better.

Advantage: Better visualization of lesion details.

Limitation: Inaccessibility to tongue lesions along the posterior half and corresponding lateral borders.

(c) Fruit Jelly Technique.

(Figs. 8.49a, b, and 8.50a-d)

- Commercially available edible "Fruit jellies" are placed in the upper and lower gingivobuccal sulcus.

- Patients are then instructed to close their mouth and ultrasound is performed.

Advantages:

- Useful in elderly and facial nerve palsy patients having difficulty holding puffed cheek for prolonged periods.

- Absence of artifacts due to vestibular air.

- Enhanced structural delineation and better evaluation of vestibular extension of lesion.

Limitation: Inability to evaluate lesions involving far posterior aspect of Vestibule. 
Fig. 8.46 Cheek layers by "Puffed Cheek" Technique.

(1) Mucosa. (2) Submucosa.

(3) Buccinator. (4) Buccal fat.

(5) Subcutaneous tissue. (6) Skin

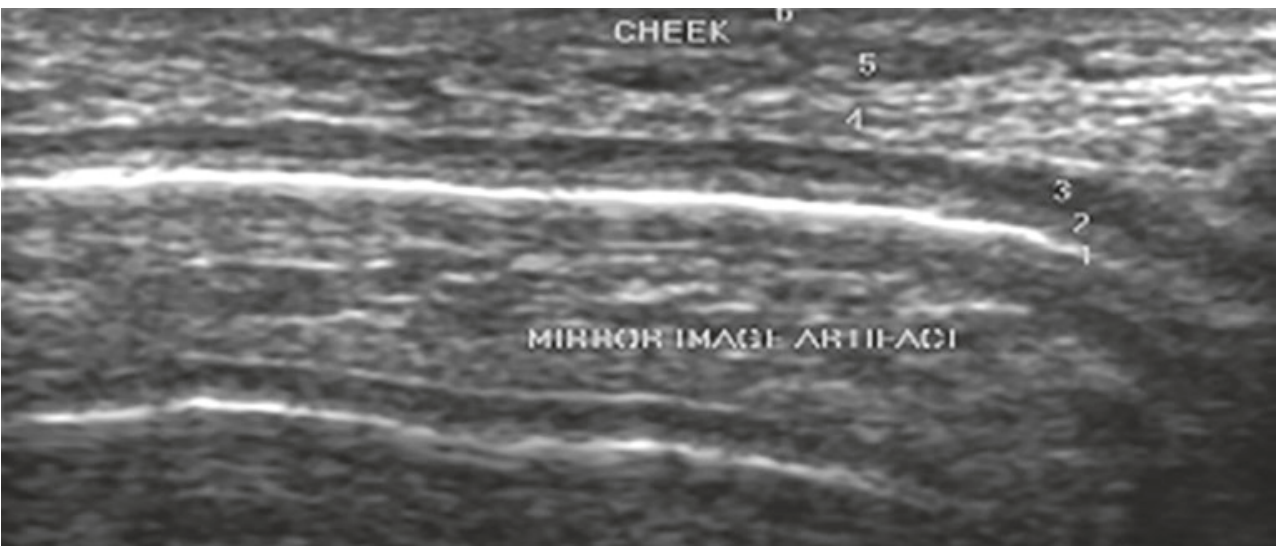

(CAssociation of Oral and Maxillofacial Surgeons of India
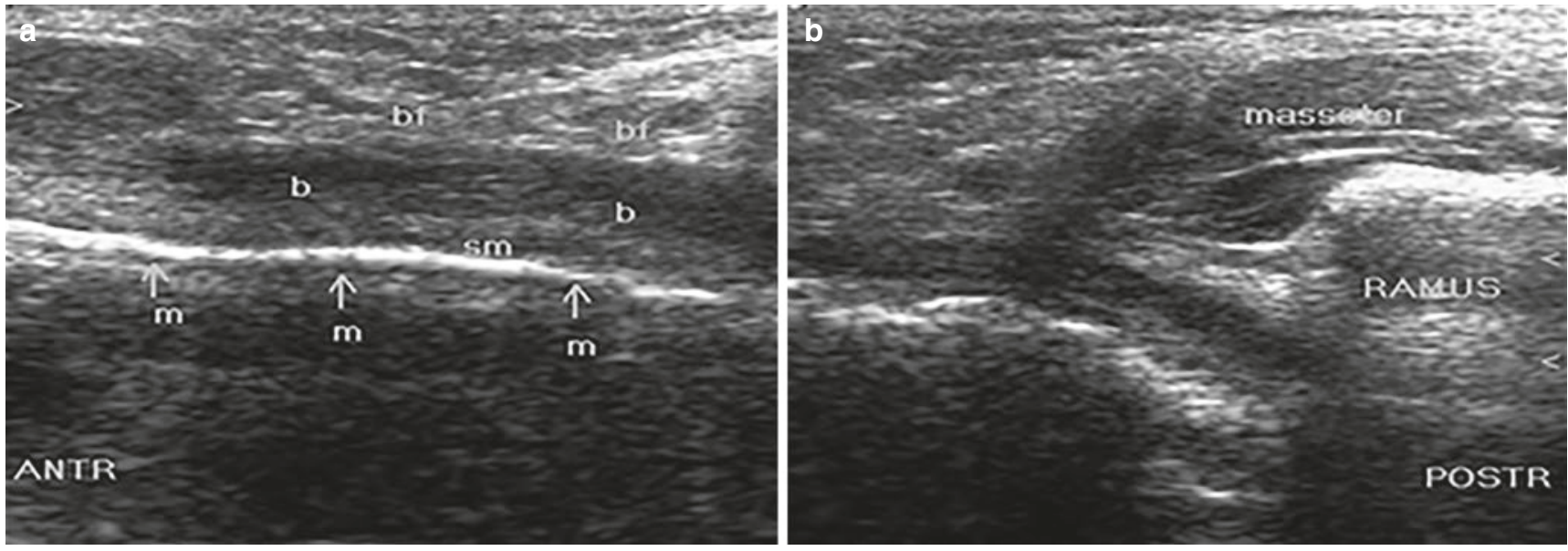

CAssociation of Oral and Maxillofacial Surgeons of India

Fig. 8.47 (a, b) Cheek layers by "Puffed Cheek" Technique. m-mucosa, sm-submucosa, b-buccinator, bf-buccal fat


CAssociation of Oral and Maxillofacial Surgeons of India

Fig. 8.48 (a), (b), and (c). "Tongue Touch" Technique. Anterior tongue, its tip and lateral borders and cheek layers 

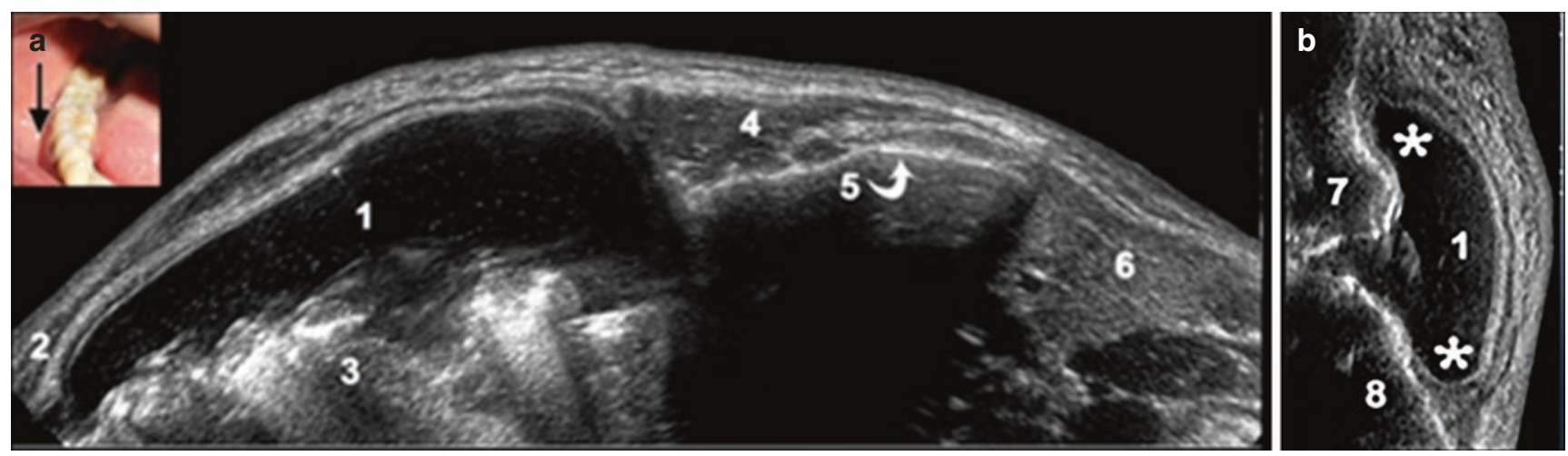

CAssociation of Oral and Maxillofacial Surgeons of India

Fig. 8.49 (a) Axial panoramic (b) Coronal Cheek layers by "Fruit Jelly" Technique. (1) Jelly (2) Angle of mouth (3) Alveolar process (4) Masseter (5) Mandible (6) Parotid (7) Maxilla (8) Mandible (Asterisk)- Upper and lower gingivobuccal sulcus
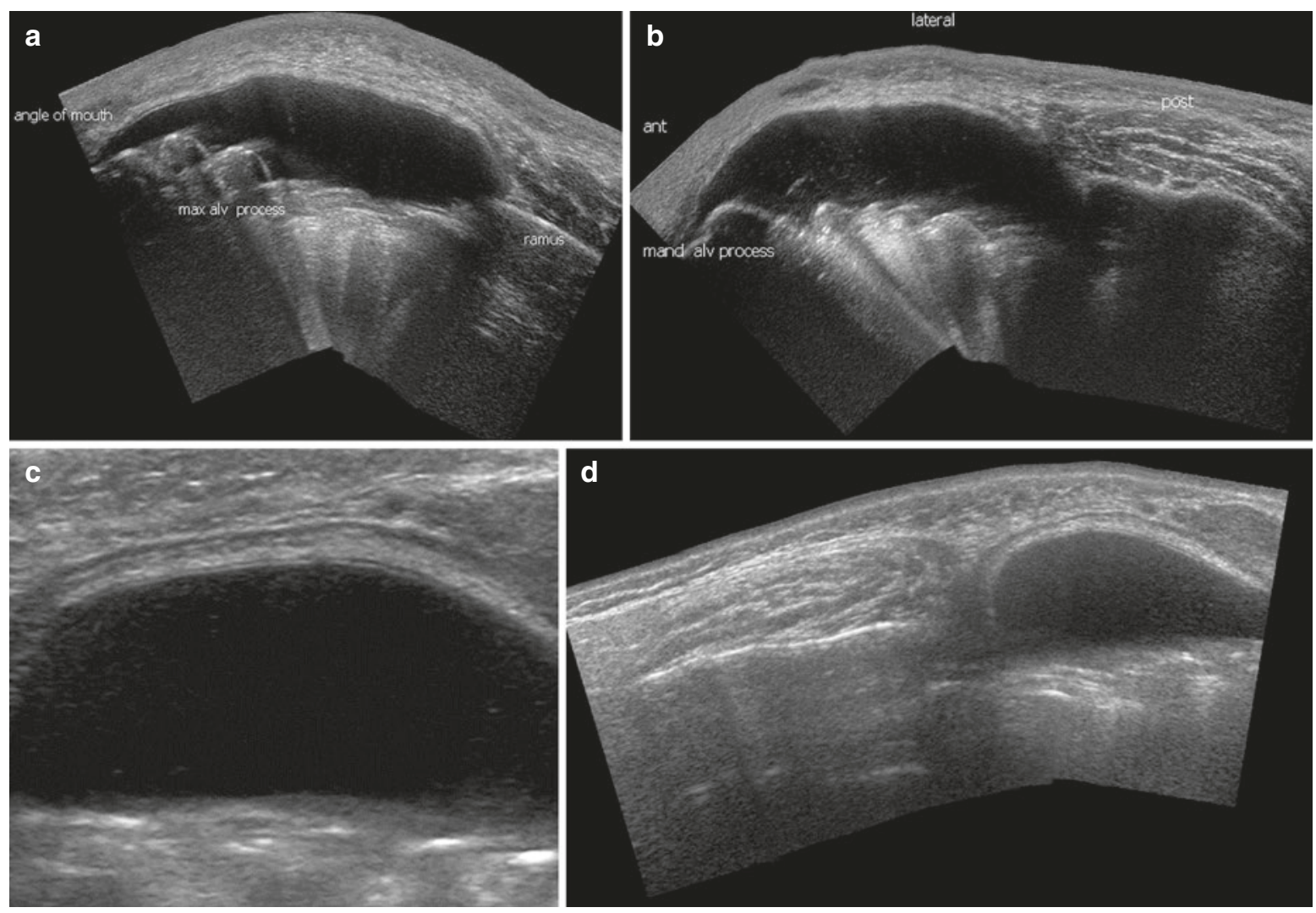

CAssociation of Oral and Maxillofacial Surgeons of India

Fig. 8.50 (a, b, c, and d).Cheek layers by "Fruit jelly" Technique. Panoramic axial slices. Coronal slices showing superior and inferior vestibules along with layers of cheek/alveolar surfaces 


\subsubsection{Case Presentation}

\section{HRUSG Cheek: Scope of Use and Limitations}

Scope of practice of HRUSG cheek includes

- Cases where limitations are posed by the clinical presentation as in case of trismus or lesions that mimic others in their clinical presentation resulting in diagnostic difficulty

- Estimation of the extent of a lesion, thus helping in tumor staging

- Cases where the use of plain radiography is limited

- Inherent drawbacks of imaging modalities like CT and MRI and their high cost

\section{Limitations}

- Structures medial to the mandibular ramus

- Difficulty in obtaining information of intact bone

- Intra-articular structures of the temporomandibular joint

- Postoperative scar tissue or a large external wound

- Very thick patients, muscles, and nerves of the face

\section{Clinical Conditions that Can Be Evaluated}

- Swollen cheek: Painful, painless, unilateral, or bilateral.

- Limited mouth opening
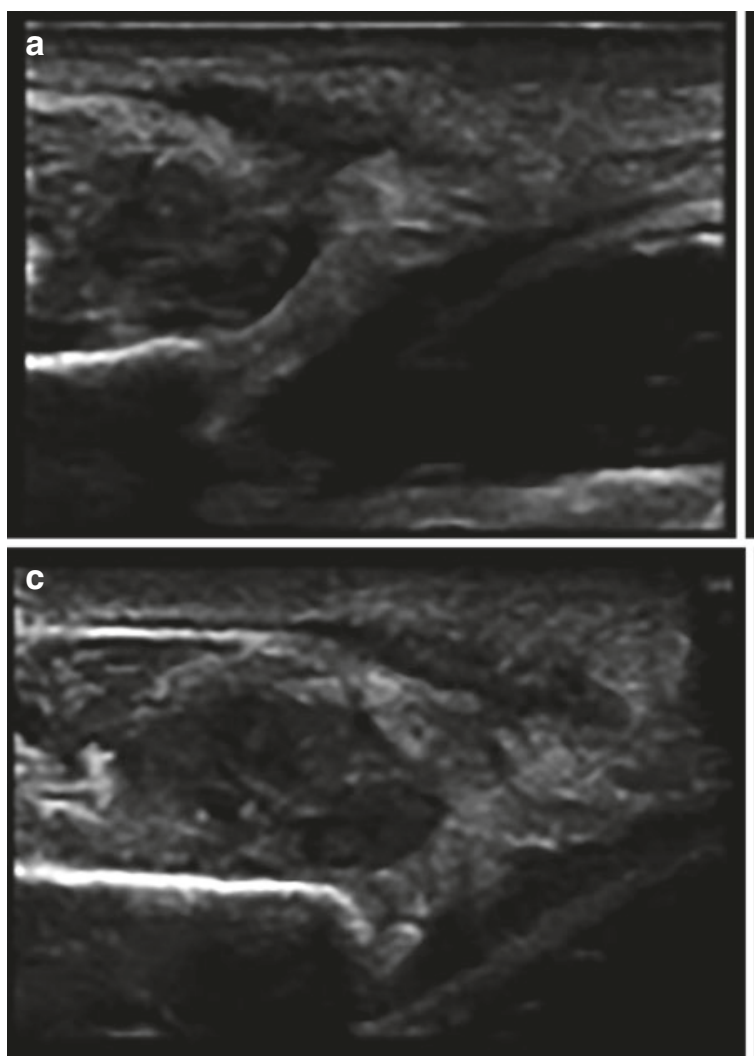

CAssociation of Oral and Maxillofacial Surgeons of India
- Painful chewing

- Swelling of the face involving the midline or parasagittal plane, floor of the mouth, TM joint region, submandibular region, nose, lip, gums, etc.

- Tongue lesions (Lateral margin).

\section{Notifiable Lesions}

- Cheek-Growth, hemangioma, lymphangioma, Oral submucous fibrosis, abscesses, discharging sinuses, etc.

- Masseter-evaluation of contraction, benign hypertrophy, trauma, Space infection, trauma, etc.

- Masticatory space.

- TMJ_-swelling, click.

- Lytic lesions of jaws.

- Gum-evaluation. Lip lesions.

- Midline swellings.

- Tumor staging.

- Salivary gland.

- Space infections.

- Tongue.

- Lymph node evaluation.

\section{Inflammatory / Infective Pathology}

(a) Cheek cellulitis with evolving abscess (Fig. 8.51a-d).
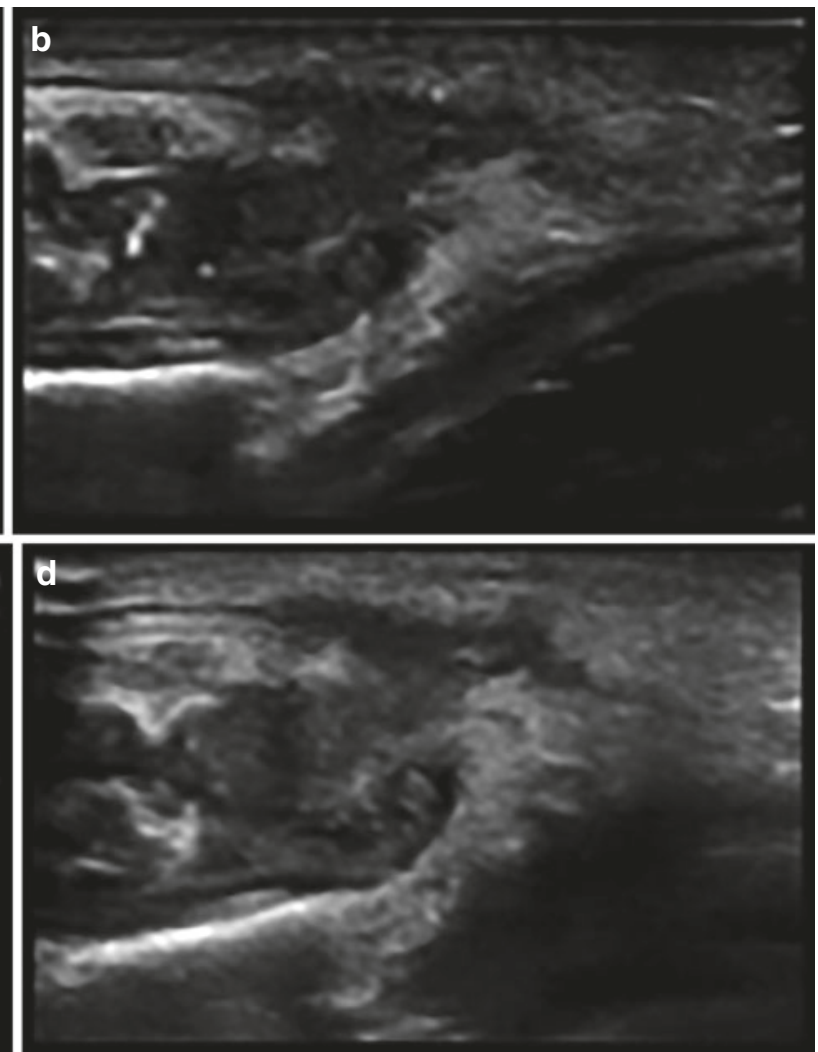

Fig. 8.51 (a, b, c, and $\mathbf{d})$.Cheek cellulitis with evolving abscess. Diffuse soft-tissue thickening, edema of deep subcutaneous fat plane, edematous buccinators and submucosal plane with mild echogenic tracking collection within the fascial planes 

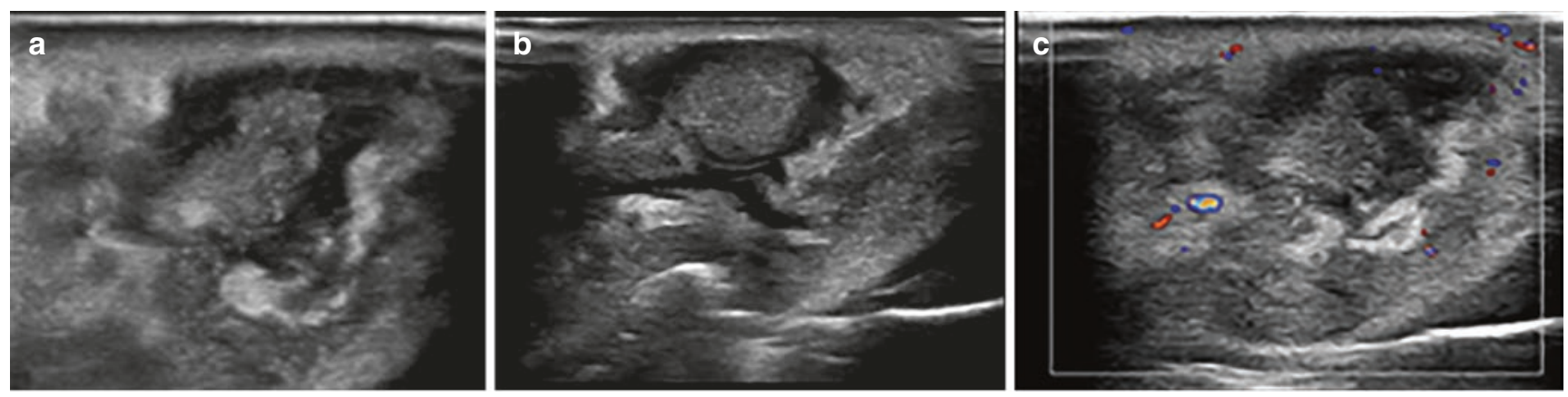

(CAssociation of Oral and Maxillofacial Surgeons of India

Fig. 8.52 (a, b and c). Cheek abscess. Focal organized thick echogenic collection in the deep subcutaneous plane with minimal extension into buccinator muscle (45 year old male, with habit of chewing betel nut)

Fig. 8.53 (a-d) Chronic osteomyelitis mandible. HRUSG showing hyperechoic lesion with hypoechoic halofungal granuloma


CAssociation of Oral and Maxillofacial Surgeons of India (b) Cheek abscess (Fig. 8.52a-c).

(c) Chronic osteomyelitis mandible (Fig. 8.53a, b).

(d) Cysticercosis parotid (Fig. 8.54).

\section{Benign Pathology.}

(a) Oral submucosal fibrosis (OSMF) (Fig. 8.55a-c).

(b) Lesion at opening of Stenson's Duct (Fig. 8.56a, b).

(c) Intraoral Mucocele (Fig. 8.57a, b).

(d) Cheek lymphangioma (Fig. 8.58a-d).

(e) Epidermoid cyst of cheek (Fig. 8.59a, b). (f) Painless unilateral swollen cheek (Intramasseteric lipoma) (Fig. 8.60).

(g) Stenson's Duct calculus (Fig. 8.61a, b).

(h) Polycystic disease of parotid (Fig. 8.62a, b).

\section{Malignant Pathology.}

(a) Buccal mucosal malignancy with intact submucosa and buccinator (Fig. 8.63).

(b) Buccal mucosal squamous cell carcinoma (Fig. 8.64 $a-c)$. 


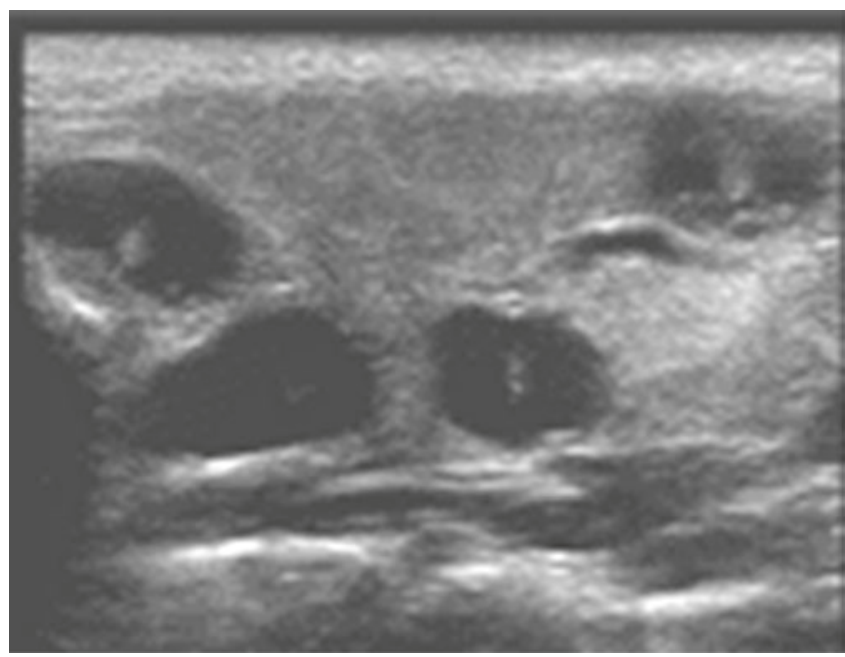

CAssociation of Oral and Maxillofacial Surgeons of India

Fig. 8.54 Cysticercosis parotid


CAssociation of Oral and Maxillofacial Surgeons of India

Fig. 8.55 (a, b and $\mathbf{c}$ ) Oral submucosal fibrosis. Axial slice showing thickening of the submucosal layer in OSMF in comparison to the normal thickness of the submucosal layer (c) Buccal mucosal carcinoma with mucosal and submucosal involvement, mandibular destruction and sparing of buccinator (Fig. 8.65).

(d) Buccal mucosal carcinoma comparative evaluation. (Fig. 8.66a, b).

(e) Superior vestibular malignant mass (Fig. 8.67a-c).

(f) Tongue malignancy-Tongue touch technique (Fig. $8.68 a, b)$.

(g) Buccal malignancy with mandibular infiltration (Fig. $8.69 a-d)$.

\section{Vascular malformations}

(a) Arteriovenous malformation cheek (Fig. 8.70a-d).

(b) Cheek hemangioma (Fig. 8.71). 

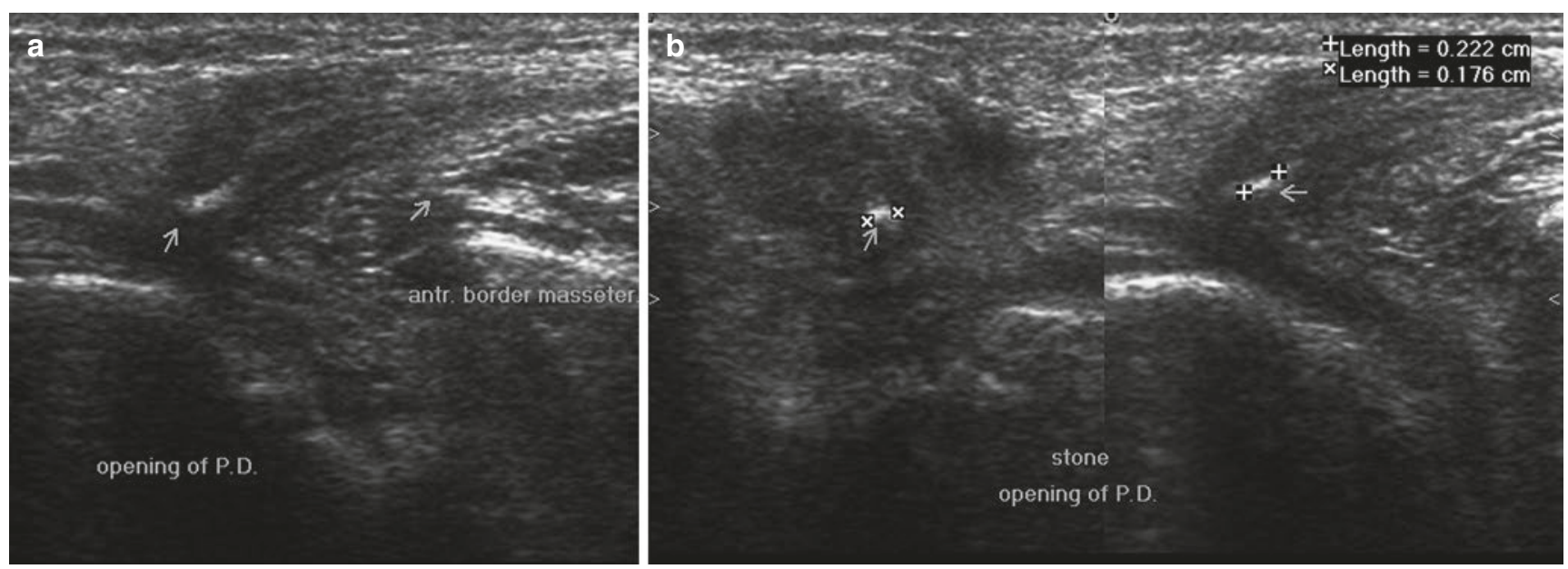

CAssociation of Oral and Maxillofacial Surgeons of India

Fig. 8.56 (a) and (b). Tiny calculus of about $1.2 \mathrm{~mm}$ revealed by HRUSG at Stenson's duct opening. (Pain while chewing food. No swelling)
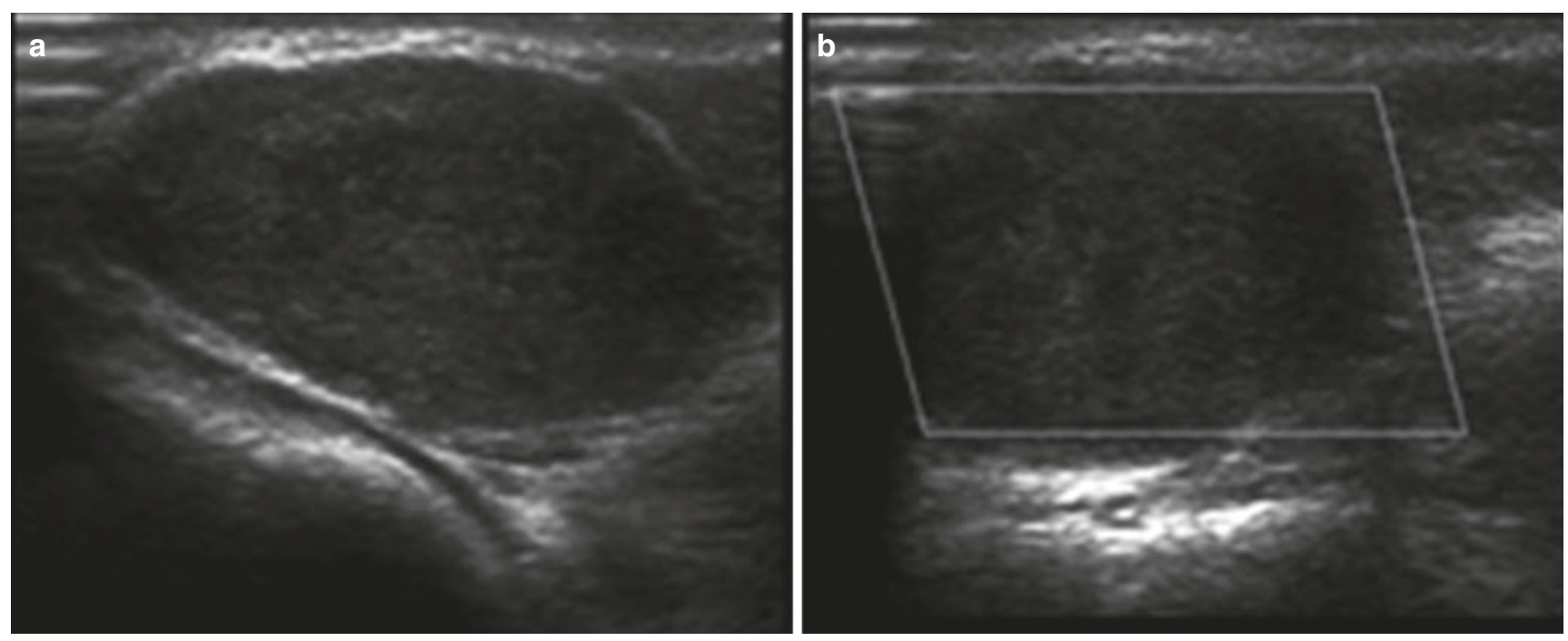

(C)Association of Oral and Maxillofacial Surgeons of India

Fig. 8.57 (a) and (b). Intraoral Mucocele. Well-defined cystic lesion in the submucosal layer with internal minimal echogenic contents. Possible diagnosis of mucocele given, later confirmed by HPR. (35 year old female, presenting with right-sided cheek swelling and mild pain) 

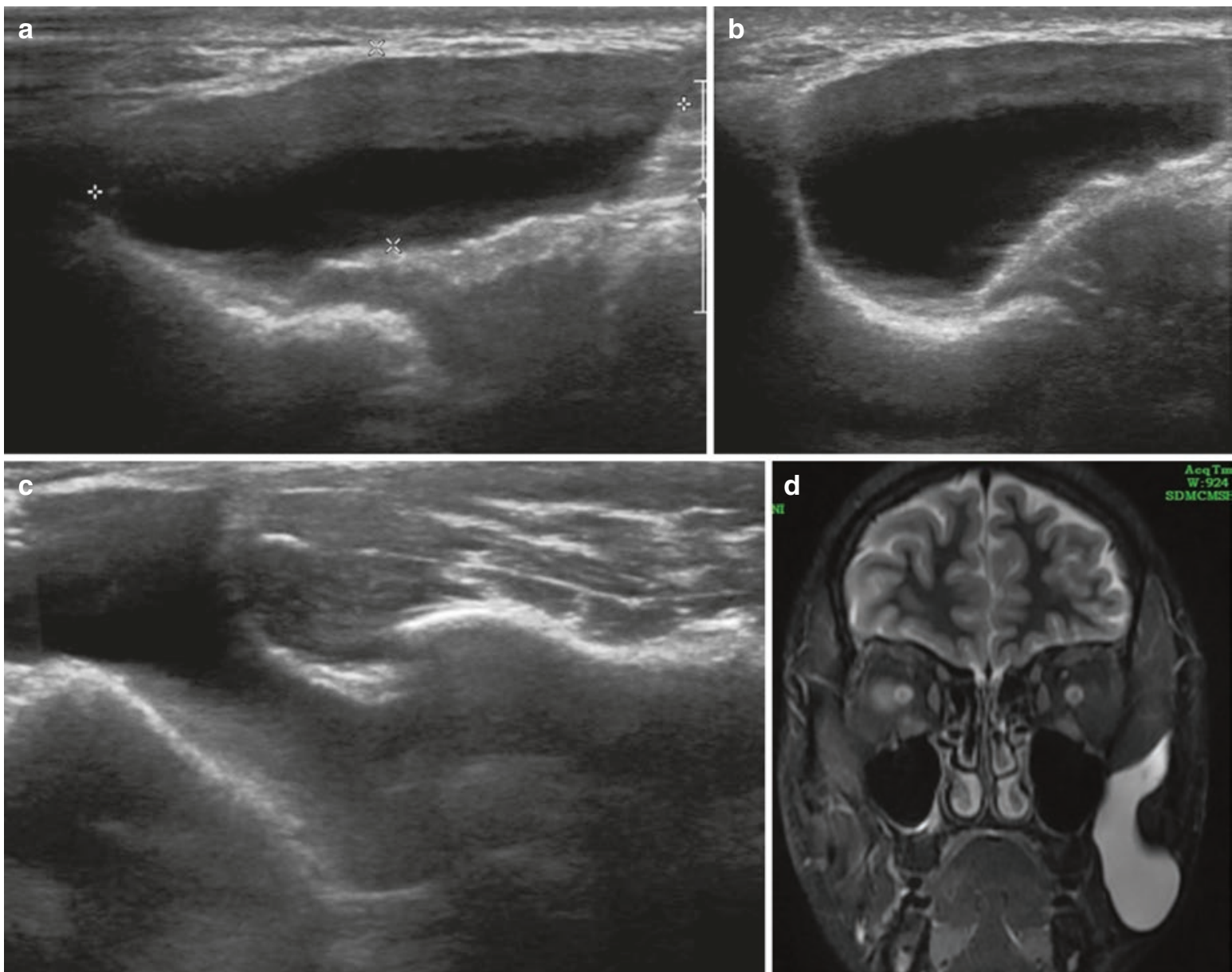

CAssociation of Oral and Maxillofacial Surgeons of India

Fig. 8.58 (a, b, c, and d). Cheek lymphangioma. Fluid-filled cystic lesion deep to submucosal layer with deep space extension adjacent to the masseter medial to mandibular ramus MRI coronal T2W imaging confirms hyperintense fluid along left side of cheek extending into infratemporal fossa (25 year old male, presenting with soft, painless swelling of left cheek)
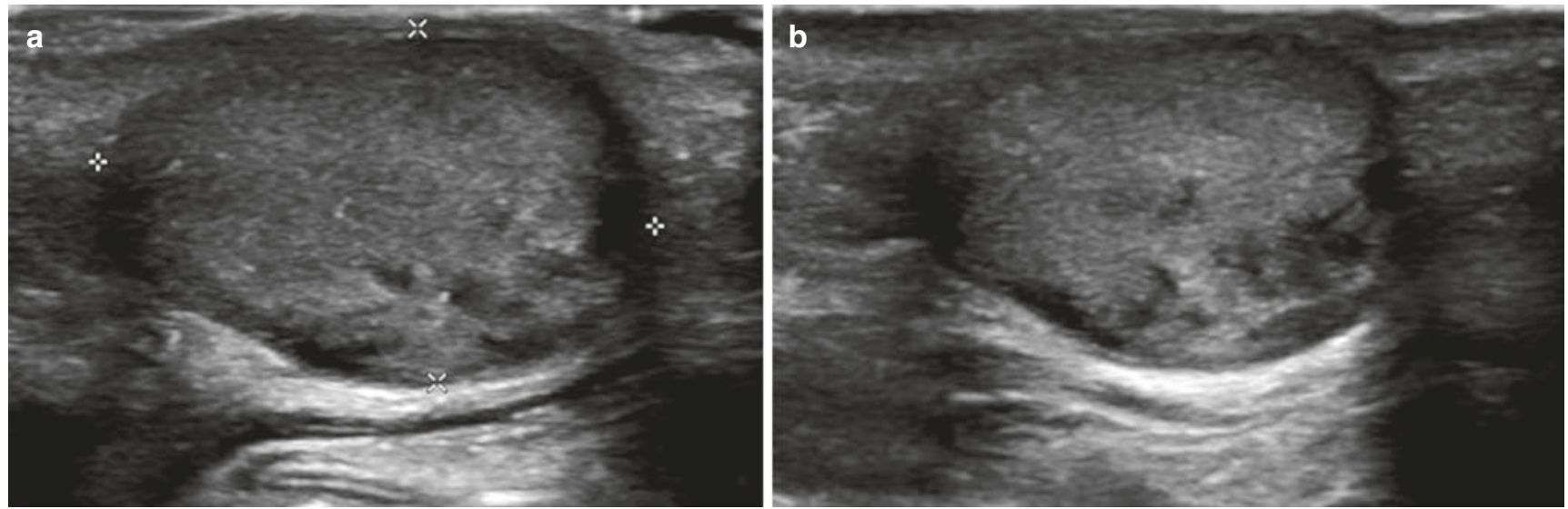

CAssociation of Oral and Maxillofacial Surgeons of India

Fig. 8.59 (a) and (b). Epidermoid cyst of cheek. Well-defined complex cystic lesion in the subcutaneous plane with internal echogenic contents causing displacement of underlying cheek layers (28 year old male presenting with painless left cheek swelling) 




CAssociation of Oral and Maxillofacial Surgeons of India

Fig. 8.60 Intramasseteric Lipoma. Elongated intramuscular lesion isoechoic to the subcutaneous fat with internal echogenic strands representing intramasseteric lipoma. (30 year old female, presenting with painless swelling of cheek)
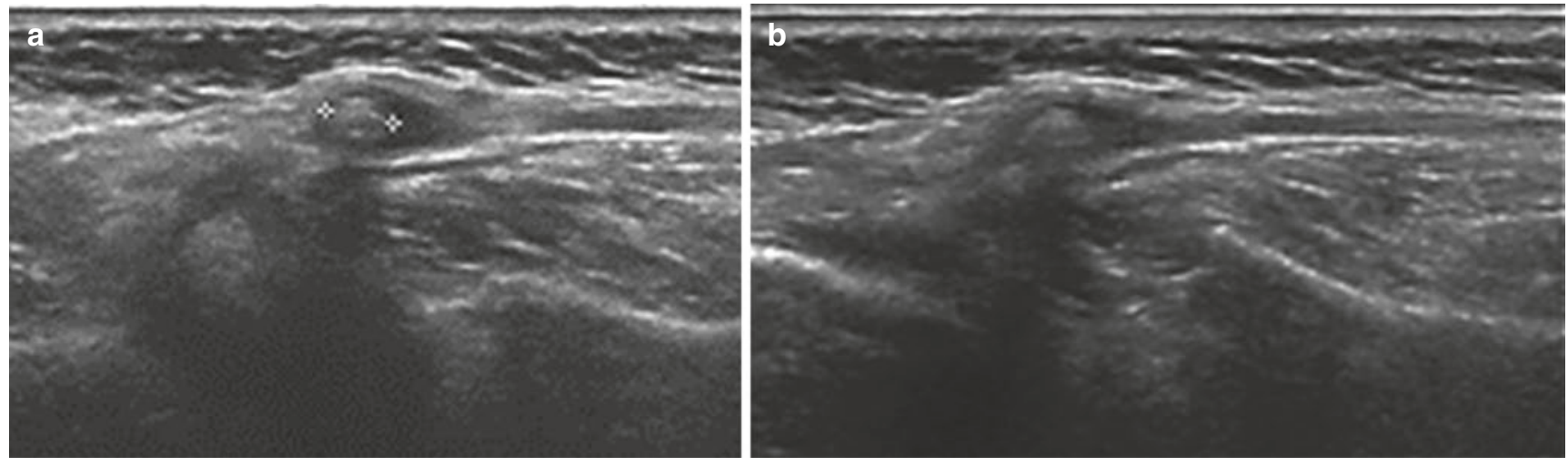

(CAssociation of Oral and Maxillofacial Surgeons of India

Fig. 8.61 (a) and (b). Stenson's duct calculus. Hyperechoic calculus in the Stenson's duct with nondilated duct proximal to the calculus (23 year old male, with history of intermittent pain on right side of the cheek)
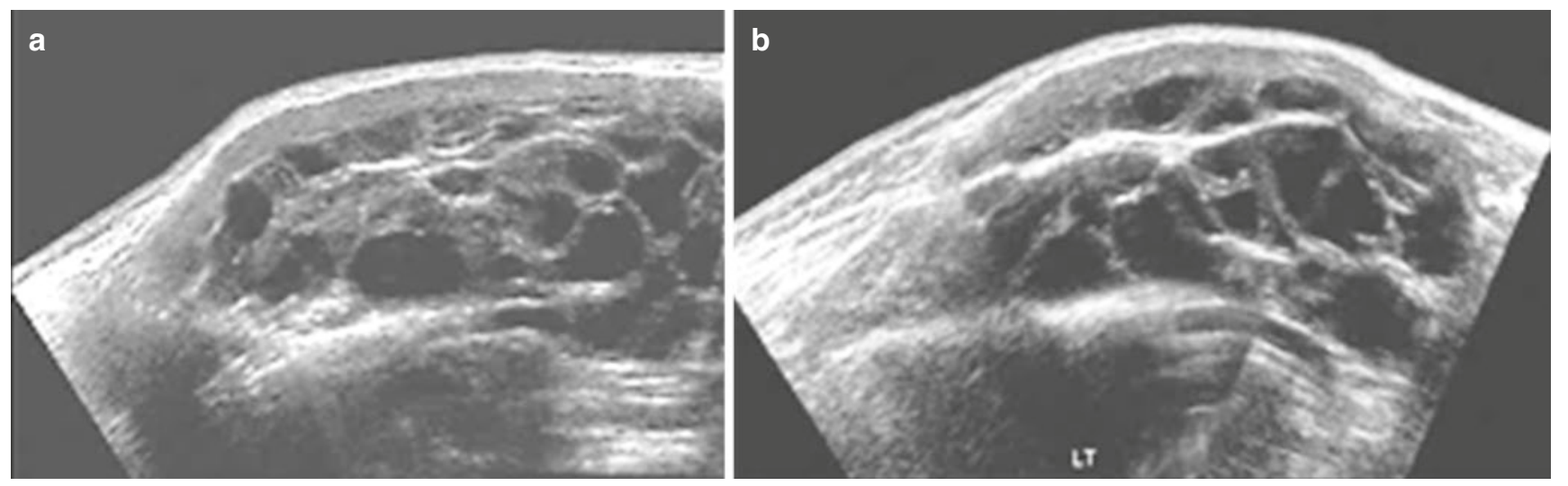

CAssociation of Oral and Maxillofacial Surgeons of India

Fig. 8.62 (a) and (b). Polycystic disease of parotid. (22 year old male Bilateral painless swollen cheek 7-8 yrs duration) 
Fig. 8.63 Buccal mucosal malignancy with intact submucosa and buccinator. Growth predominantly involving the mucosa, distinct from submucosa (between asterisk) buccinator (star) appears normal. (55 year old male with irritating nonhealing ulcer left cheek)

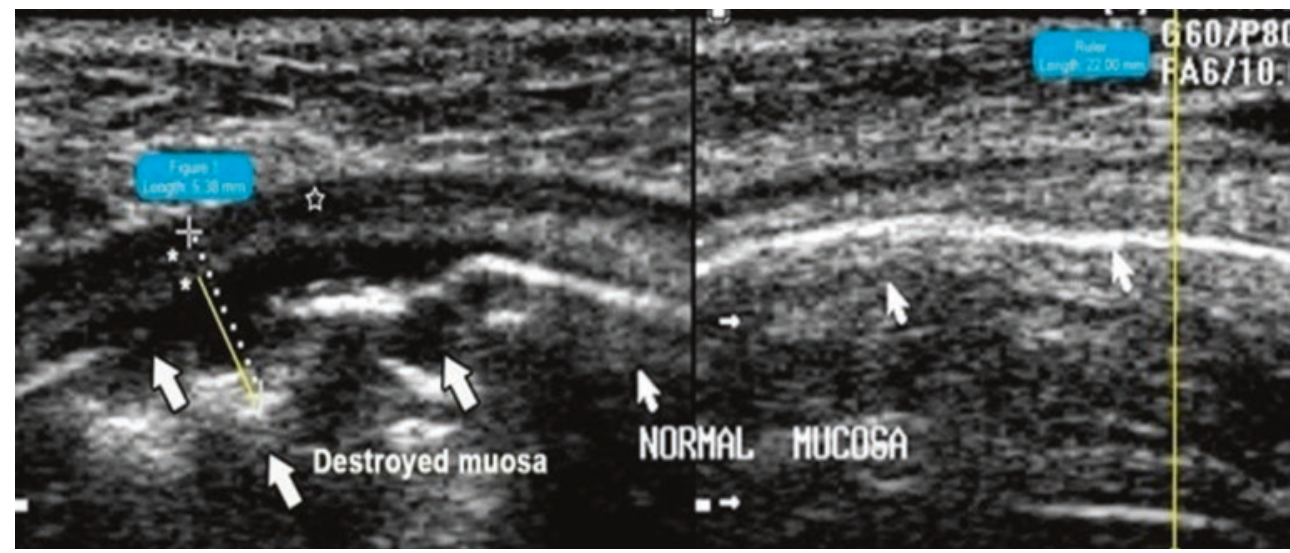

CAssociation of Oral and Maxillofacial Surgeons of India
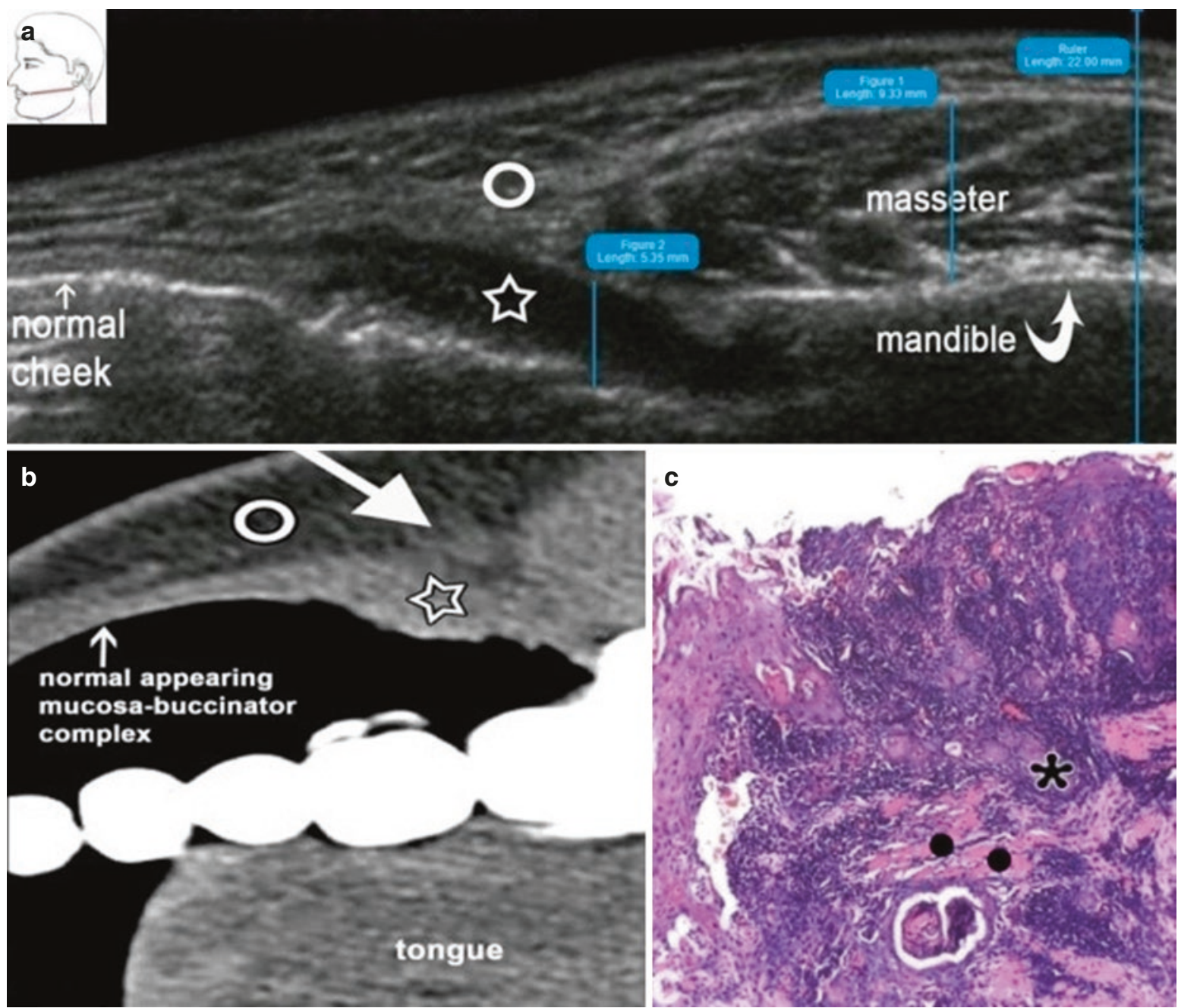

(CAssociation of Oral and Maxillofacial Surgeons of India

Fig. 8.64 (a, b, and $\mathbf{c})$. Early Carcinoma of the cheek better seen on HRUSG than by CT slice at the same level. Proved by Histopathology (52 year old male with squamous cell carcinoma of the buccal mucosa) 


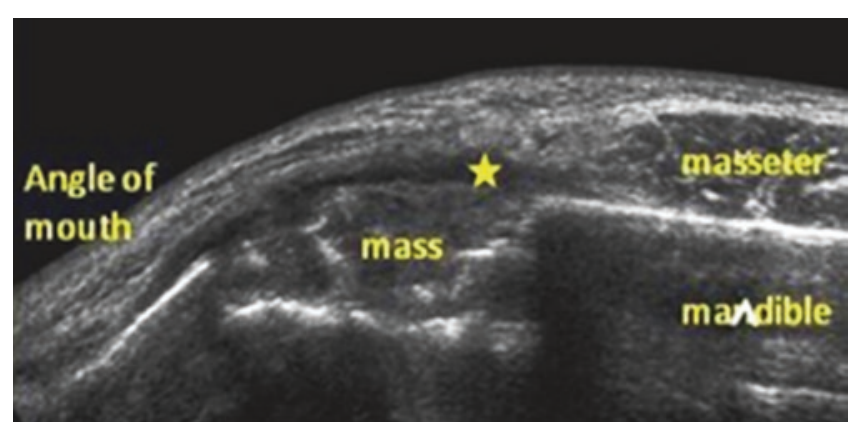

CAssociation of Oral and Maxillofacial Surgeons of India

Fig. 8.65 Buccal mucosal carcinoma

Fig. 8.66 (a) Buccal mucosal carcinoma (Resting phase). (b) Buccal mucosal carcinoma (Fruit jelly technique). Patient was unable to perform the puffed cheek technique. Mass involving the mucosa, submucosa, buccinator, and fat
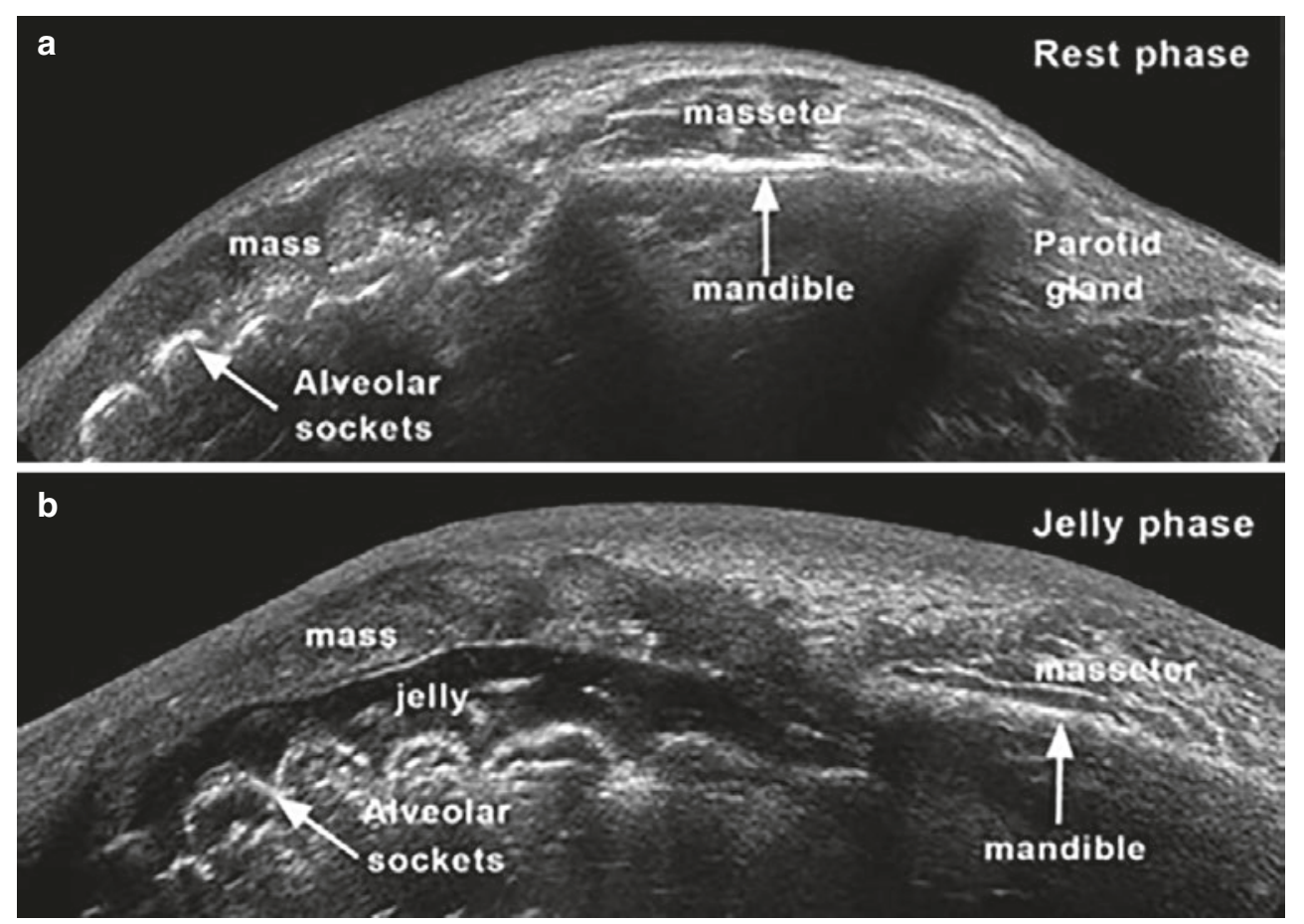

(CAssociation of Oral and Maxillofacial Surgeons of India 
Fig. 8.67 (a, b and c).

Superior vestibular malignant mass. Coronal sections revealing predominantly hypoechoic, superior vestibular mass with irregular margins extending into cheek layers and causing underlying bony destruction. Comparison between (a) neutral cheek,

(b) fruit jelly Technique and

(c) $\mathrm{CT}$
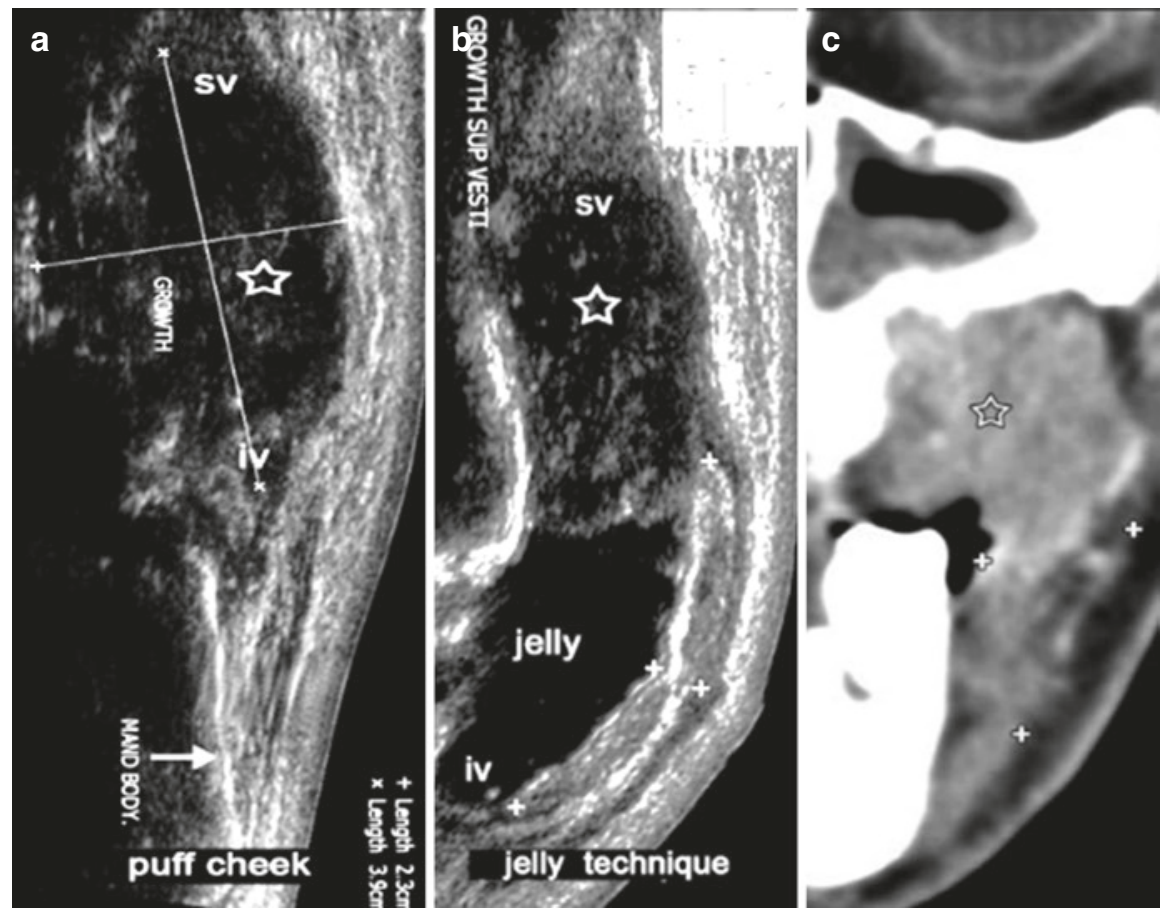

CAssociation of Oral and Maxillofacial Surgeons of India


CAssociation of Oral and Maxillofacial Surgeons of India

Fig. 8.68 (a) and (b). Tongue malignancy against cheek wall. Irregular lesion along the Right anterolateral margin of the tongue by "Tongue touch" technique. Few enlarged lymph nodes are also noted with loss of hilar anatomy representing metastatic lymph nodes. (40 year old male, with complaint of swelling over the tongue) 

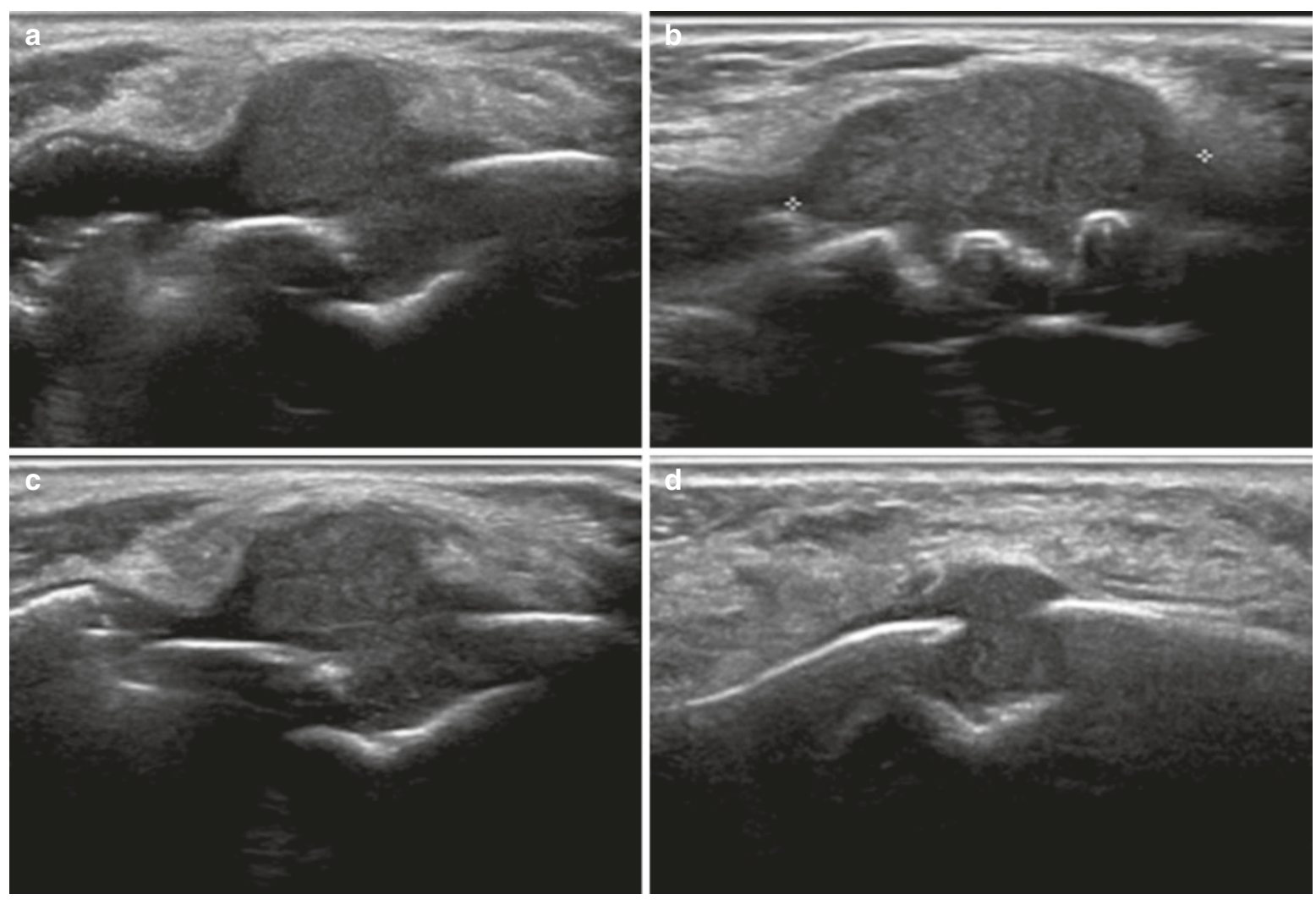

CAssociation of Oral and Maxillofacial Surgeons of India

Fig. 8.69 (a, b, c, and d) Buccal malignancy with mandibular infiltration. Heterogeneous mass lesion along the buccal mucosa extending into lower buccogingival sulcus with infiltration into submucosa and buccinator muscle. Focal cortical break is also noted in the mandibular bone with intraosseous infiltration. (48 year old male tobacco chewer presenting with right cheek swelling)


(CAssociation of Oral and Maxillofacial Surgeons of India

Fig. 8.70 (a, b, c, and d) Arteriovenous malformation (AVM) cheek. Focal soft-tissue thickening with internal hypoechoic areas. Doppler study shows multiple vascular channels with high velocity, low resis-

tance flow suggestive of AVM. (40 year old female presenting with history of swelling and intermittent pain) 
Fig. 8.71 Cheek

Hemangioma. Mixed echoic hemangioma with phlebolith. Absence of flow signals due to low-velocity flow

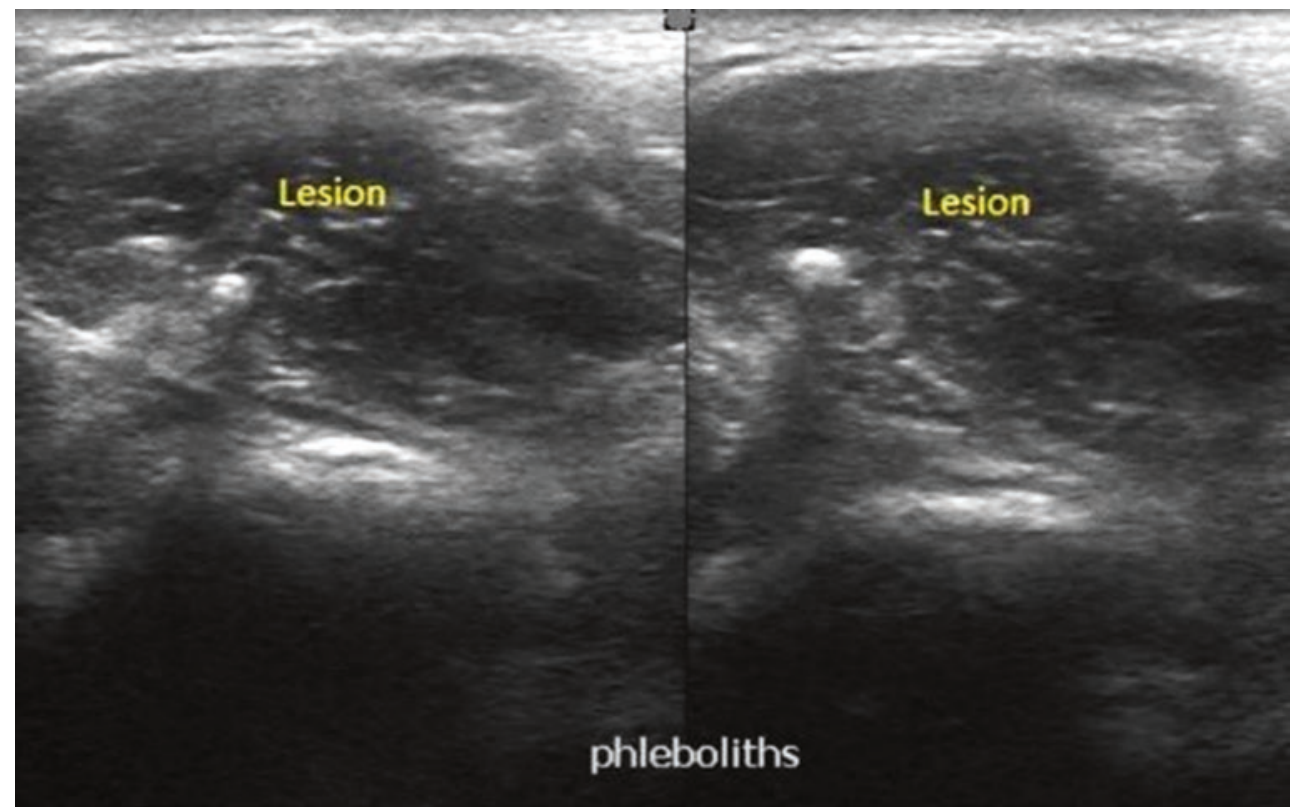

(C)Association of Oral and Maxillofacial Surgeons of India

\subsection{Conclusion}

It is extremely difficult to limit radiology to a chapter and what has been written does not represent the whole of maxillofacial radiology. Only the relevant aspects and the bare minimum essentials of radiology for maxillofacial surgeons have been covered in this chapter. The entire range of radiology for maxillofacial surgeons, dealing with surgical problems pertinent to the maxillofacial region, would require more expansive and elaborate writing.

This chapter on radiology provides an outlook to the surgeon, who can proceed to applying radiology in his everyday surgical practice. In the event of encountering any lacunae in the book, the MFS must refer to the textbooks dedicated to maxillofacial radiology. Being a surgeon, the MFS should be aware of the use of the chest radiograph and its applications in his practice. The role of high-resolution ultrasonography has also been emphasized in this chapter. Dental radiology, both extra and intraoral, has not been included since all maxillofacial surgeons have extensive knowledge of the same in their surgical practice.

Since it is the MFS who is acquainted with the patient's clinical status, he must properly plan the imaging pathway for the patient. It is for this purpose that the MFS should be aware of the various imaging modalities as regards their nature, mode of image formation, their usefulness over other modalities, and their drawbacks. Radiation safety and cost effectiveness of a specific imaging modality also need to be kept in mind. The imaging method selected should be one that provides the required information within the shortest time possible, be the safest option for the patient, and be the most economical, while at the same time providing maximum information.

The MFS can commence with simple basic imaging methods like plain radiography or USG and later proceed to higher imaging methods in case the basic methods were not enough to satisfy the surgeon's needs for patient management. A recent costlier imaging method does not necessarily mean it is the best. Many times, it is the simpler basic imaging methods that provide more crucial findings that help in understanding complex disease processes.

Acknowledgments The authors would like to acknowledge \& thank Dr Niranjan Kumar, M.Ch FRCS. ; Honorable Vice-Chancellor, Shri Dharmasthala Manjunatheshwara University for his valuable constructive suggestions and constant support towards this endeavour. 


\section{References}

1. Fusaro F, Constantinides F, Maglione M, Dalessadri D, Vettori E, Lenarda RDI. Comparison of orthopantomography (OPG) and computed tomography (CT) accuracy in predicting inferior alveolar nerve injury following lower third molar surgical extraction [Internet]. 2017 [cited 2019Jun3]. Available from: https://www. researchgate.net/publication/319548814_Comparison_of_orthopantomography_OPG_and_computed_tomography_CT_accuracy_ in_predicting_inferior_alveolar_nerve_injury_following_lower_ third_molar_surgical_extraction

2. Abramovitch K, Rice DD. Basic principles of cone beam computed tomography. Dent Clin N Am. 2014;58(3):463-84.

3. Lucas J.. What are gamma-rays? Available from: https://www. livescience.com/50215-gamma-rays.html

4. Ferreira RI, de Almeida SM, Boscolo FN, Santos AO, Camargo EE. Bone scintigraphy as an adjunct for the diagnosis of oral diseases. J Dent Educ. 2002;66(12):1381-7.

5. Appadurai R, Lingeshwar D, Venugopal S, Christina RD. Bone scintigraphy as a diagnostic tool in condylar hyperplasia [Internet]. 2017 [cited 2019Jun3]. Available from: http://www.joomr.org/article. asp? issn $=2321-3841$; year $=2017$; volume $=5$;issue $=3$; spage $=84$; page $=89 ;$ aulast $=$ Appadurai

6. Griffeth LK. Use of PET/CT scanning in cancer patients: technical and practical considerations [Internet]. Proceedings (Baylor University. Medical Center). Baylor Health Care System; 2005 [cited 2019Jun4]. Available from: https://www.ncbi.nlm.nih.gov/ pmc/articles/PMC1255942/

7. Williams N, O'Connell PR, McCaskie AW. Bailey and Love's short practice of surgery. 27th ed. New York: CRC Press; 2018.

8. Pooley RA. AAPM/RSNA Physics Tutorial for Residents Fundamental Physics of MR Imaging [Internet]. 2005 [cited 2019Jun3]. Available from: https://pubs.rsna.org/doi/pdf/10.1148/ rg. 254055027
9. Singh GP, Dogra S, Kumari E. Ultrasonography: maxillofacial applications. Annals of Dental Specialty. 2014;2(3):104-7.

10. Kumar V, Abbas AK, Aster JC. Robbins and Cotran pathologic basis of disease. 9th ed. Philadelphia: W.B. Saunders; 2015.

11. Fraser RS, Muller NL, Colman N, Pare PD. Fraser and Pare's diagnosis of diseases of the chest. 4th ed. Philadelphia: W.B. Saunders; 1999.

12. Christopoulos A. Mouth Anatomy [Internet]. Overview, Gross Anatomy: Oral Vestibule, Gross Anatomy: Oral Cavity Proper. 2015 [cited 2019Jun5].

13. Surgical fascial spaces [Internet]. online presentation [cited 2019 Jun 7];Available from: https://en.ppt-online.org/369373

14. La'Porte SJ, Juttla JK, Lingam RK. Imaging the Floor of the Mouth and the Sublingual Space [Internet]. RadioGraphics. 2011 [cited 2019Jun5]. Available from: https://pubs.rsna.org/doi/full/10.1148/ rg.315105062

15. Joshi SK, Kamalapur MG, Joshi AS, Hallikeri K. Ultrasonographic evaluation of carcinoma of buccal mucosa: Ultrasound technique and evaluation of neoplastic conditions. J. Evid. Based Med. Healthc. 2016;3(34):1649-55.

\section{Further Reading}

Mosier K. Functional imaging of swallowing. In: Mukherji SK, Casterlijins JA, editors. Head and neck imaging. Berlin: Springer; 2000. p. 79-86.

Yousem DM (2000) Magnetization transfer imaging of the Extracranial head and neck. In: Mukherji SK, Casterlijins JA (eds). Modern head and neck imaging. Springer, Berlin, pp1-16.

Sanjay N. Jain (2019) a Pictoral essay: radiology lines and tubes in the intensive care unit. Indian J Radiol Imaging. 2011;21:182-90.

Open Access This chapter is licensed under the terms of the Creative Commons Attribution 4.0 International License (http://creativecommons. org/licenses/by/4.0/), which permits use, sharing, adaptation, distribution and reproduction in any medium or format, as long as you give appropriate credit to the original author(s) and the source, provide a link to the Creative Commons license and indicate if changes were made.

The images or other third party material in this chapter are included in the chapter's Creative Commons license, unless indicated otherwise in a credit line to the material. If material is not included in the chapter's Creative Commons license and your intended use is not permitted by statutory regulation or exceeds the permitted use, you will need to obtain permission directly from the copyright holder. 